\title{
Development of methods for the detection of chemical and biological warfare agents
}

\author{
Megan Rea DeJesus \\ West Virginia University
}

Follow this and additional works at: https://researchrepository.wvu.edu/etd

\section{Recommended Citation}

DeJesus, Megan Rea, "Development of methods for the detection of chemical and biological warfare agents" (2013). Graduate Theses, Dissertations, and Problem Reports. 473.

https://researchrepository.wvu.edu/etd/473

This Dissertation is protected by copyright and/or related rights. It has been brought to you by the The Research Repository @ WVU with permission from the rights-holder(s). You are free to use this Dissertation in any way that is permitted by the copyright and related rights legislation that applies to your use. For other uses you must obtain permission from the rights-holder(s) directly, unless additional rights are indicated by a Creative Commons license in the record and/ or on the work itself. This Dissertation has been accepted for inclusion in WVU Graduate Theses, Dissertations, and Problem Reports collection by an authorized administrator of The Research Repository @ WVU.

For more information, please contact researchrepository@mail.wvu.edu. 


\title{
Development of Methods for the Detection of Chemical
} and Biological Warfare Agents

\author{
Megan Rea DeJesus \\ Dissertation submitted to the \\ Eberly College of Arts and Sciencies \\ at West Virginia University \\ in partial fulfillment of the requirements \\ for the degree of \\ Doctor of Philosophy \\ in \\ Chemistry \\ Approved by \\ Fred L. King, Ph.D., Chair \\ Patrick S. Callery, Ph.D. \\ Lisa A. Holland, Ph.D. \\ Glen P. Jackson, Ph.D. \\ Ronald B. Smart, Ph.D. \\ C. Eugene Bennett Department of Chemistry \\ Morgantown, West Virginia \\ 2013
}

Keywords: Gas Chromatography, Glow Discharge, Time-of-Flight, Mass Spectrometry, Microbial Forensics Copyright 2013 Megan Rea DeJesus 


\title{
ABSTRACT \\ Development of Methods for the Detection of Chemical and Biological Warfare Agents
}

\author{
Megan Rea DeJesus
}

This dissertation sought to find conditions that enabled the characterization of weapons of mass destruction, be that chemical, explosives, or biological, and find unique ion signals for those materials. Chapter 2 examines how to improve the analytical capability of the pulsed glow discharge through an increased understanding of the ionization processes inherent to the technique. Results of a parametric evaluation of ionization processes in the plasma demonstrated that within the glow discharge ion source there are conditions that can be determined which will enhance the signal of the analytical ion. A key innovation was the determination, that in constant power operations, optimal analyte signals could be found $6-8 \mathrm{~mm}$ in distance from the cathode and at shorter pulse widths and duty cycles. For the first time, the behavior of argon doubly charged species was characterized in these pulsed plasmas. Whereas the goal of Chapter 2 was to understand the fundamental characteristics of the pulsed glow discharge, Chapters 3 and 4 strive to expand its future possibilities through the coupling of gas chromatography and the pulsed glow discharge ion source to achieve chemical speciation. Chemical speciation can be achieved through structural information from the plateau region and molecular ion information from the afterpeak region and both can be acquired simultaneously. The ability of the pulsed glow discharge to acquire both pieces of information gives the analyst a greater degree of confidence in the identification of the compound; no other technique is capable of providing both pieces of information simultaneously. The purpose of these studies was to determine if the time-gated pulsed glow discharge coupled with gas chromatography mass spectrometry could provide adequate information to detect a chemical warfare agent metabolite or an explosive related compound. We were able to demonstrate that the pulsed glow discharge provides structural information during the plateau for the analytes and we learned that it was important to control the analyte concentration introduced to the plasma so that it does not quench the afterpeak signal. Future directions would focus on lowering the analyte concentration sufficiently. In Chapter 5, we hypothesized that the separation of particles by size would enhance the ability to discriminate between different sources of a Bacillus anthracis surrogate. Size selection was combined with analytical techniques to enhance the capability of identifying biological signatures of bacterial spores. It was found 
that size separation permitted a more rapid determination by SEM to confirm the presence of spores, but did not enhance the ability of Raman to identify the spores. Ultimately, results from these analyses can be used to build a library to determine an organism's unique biological signature that can be correlated with known growth and processing methods to identify how, when, and where the sample was produced. 
Lovingly dedicated to the memory of Romeo P. de Jesus, my father, who made all of my dreams possibilities. 
Education is not the filling of a pail, but the lighting of a fire.

William Butler Yeats

\section{Acknowledgements}

Every significant achievement is the product of a collaborative effort. A profound "thank you" to those that contributed to the completion of this dissertation. At the top of my list of acknowledgements I express my deepest appreciation to my doctoral mentor, Dr. Fred King, for offering me the opportunity to study at West Virginia University and for sponsoring my research on glow discharge mass spectrometry. For patient guidance, thoughtful advice, admirable selflessness, and steadfast encouragement over numerous years, I am eternally indebted. He is a true role model and inspiration.

My heartfelt thanks goes to Dr. Ronald Smart for introducing this former Textiles, Apparel, and Merchandising major to the world of research chemistry and to Dr. Carol Babyak for taking me under her wing and providing me with the foundation for becoming an analytical research chemist.

I extend my gratitude to the rest of my doctoral committee: Dr. Callery, Dr. Holland, and Dr. Showalter for feedback and encouragement. I acknowledge Dr. Jackson, who, without hesitation, had agreed to witness this endeavor to fruition. Thank you.

None of this would have been possible without the support of Dr. Cris Lewis and Dr. James H. Barnes, IV who, in conjunction with Dr. King, invited me to conduct research at Los Alamos National Laboratories. Moreover, I thank Cris and James for the valuable gift of time and attention in which I was able to grow as a research chemist. In addition, my thanks goes to Aron Pickering and Jordan Schoonover for assistance in lab, and to Rodney McCabe for help with the SEM micrographs. My internship at Los Alamos was a truly rewarding research experience enriched by the friendship of fellow students from all over the world.

The excellent engineering and technical assistance from Don Feathers, Allen Burns, Phillip Tucker, and J.R. Taylor is gratefully acknowledged.

I will forever appreciate the support and friendship of my fellow King group members and WVU chemistry colleagues: Yuchen Lu, Jennifer Robertson-Honecker, Na Zhang, Ting Zhao, Guodong Gu, Teerapat Rojsajjakul, Han Wang, Alexandria Pavkovich, Andrea Kravats, Kimberly (Fisher) Quedado, Jeremiah Hubbard, Grissell Carrero Martinez, Arica Jordan, and many moreMorgantown has become my home, and the people of the WVU chemistry department, my family. I thank you. I am especially grateful to Teerapat and Kimberly for reviewing the manuscript in spite of very busy schedules. Their suggestions have, without a doubt, enhanced this final document. As if I were completing a marathon, they have propelled me to the finish line.

For the helpful comments, insightful discussions, and unwavering support (and also the use of his laptop computer), I thank Eric Loomis.

I am fortunate to work with a team of intelligent scientists at MRIGlobal, who provided feedback on my oral presentation. I am endlessly grateful for the confidence they gave me to complete this journey.

With love and gratitude, I thank my enthusiastic team of cheerleaders, aka 'Team Chemistry': Eileen 'Mom' and Tom Wally, the Calmbachers-Joni, Cory, Kara, and Kylie, Clare 'M\&M' and Lamar 'Grandad' Farrel, Aunt Joce and Daniel Paulus, and Aunt Gina and Mick Stohr. 


\section{Contents}

$\begin{array}{lll}1 & \text { Introduction } & 1\end{array}$

1.1 Mass Spectrometry . . . . . . . . . . . . . . . . . . . 2

1.2 Ion Source: Glow Discharge . . . . . . . . . . . . . . . . . . . 3

$1.2 .1 \quad$ Establishment of a Glow Discharge . . . . . . . . . . . . . . . 4

1.2 .2 Spatial Regions of a Glow Discharge . . . . . . . . . . . . . . 6

1.2 .3 Cathodic Sputtering . . . . . . . . . . . . . . 6

1.2 .4 Excitation and Ionization Processes . . . . . . . . . . . . . . . 9

1.2 .5 Pulsing of the Glow Discharge . . . . . . . . . . . . . . . . . . . . . . . 11

1.2 .5 .1 The Prepeak . . . . . . . . . . . . . . . 12

1.2.5.2 The Plateau . . . . . . . . . . . . . . . . . . . . . . 12

1.2 .5 .3 The Afterpeak . . . . . . . . . . . . . . . . 14

1.2 .6 Interface/Vacuum System . . . . . . . . . . . . . . . . . . 15

1.3 Mass Analyzer: Time-of-Flight Mass Spectrometer. . . . . . . . . . . 16

1.3 .1 Time-of-Flight Fundamentals . . . . . . . . . . . . . . . . . 18

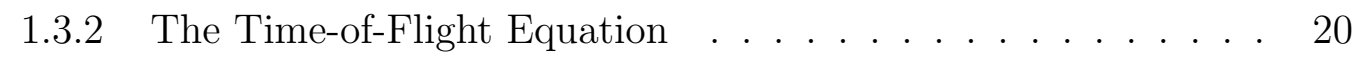

$1.3 .3 \quad$ Calibrating the Mass Spectrum . . . . . . . . . . . . . . . . 21

1.3.4 Resolution . . . . . . . . . . . . . . . . . . 21

1.4 Detector: Microchannel Plate Detector . . . . . . . . . . . . . . . . . 22

1.5 Gas Chromatograph . . . . . . . . . . . . . . . . . . . 24

1.6 Solid Phase Microextraction . . . . . . . . . . . . . . . . . . . . . . 26

1.7 Pycnometer . . . . . . . . . . . . . . . . . . . . . 27

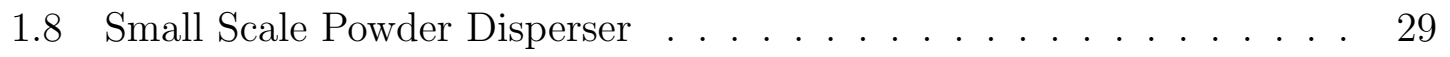

1.9 Aerodynamic Particle Sizer . . . . . . . . . . . . . . . . . . . . . . . 31

1.10 Micro-Orifice Uniform Deposit Impactor . . . . . . . . . . . . . . . . 32

1.11 Scanning Electron Microscope . . . . . . . . . . . . . . . . . . . . . 34

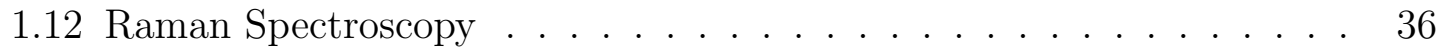

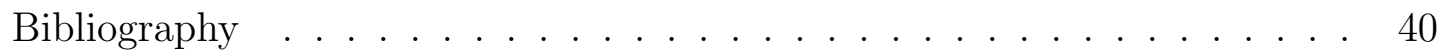


2 Ion Formation in Millisecond Pulsed Glow Discharge Plasmas 48

2.1 Abstract . . . . . . . . . . . . . . . . . . . . . 48

2.2 Introduction . . . . . . . . . . . . . . . . . . . . . . . . . . . 49

2.3 Materials and methods . . . . . . . . . . . . . . . . . . . 50

2.3 .1 Glow discharge ion source . . . . . . . . . . . . . . . . 50

2.3 .2 Time of flight mass analyzer . . . . . . . . . . . . . . . . . 51

2.3 .3 Data collection . . . . . . . . . . . . . . . 53

2.4 Results and discussion . . . . . . . . . . . . . . . . . . . 56

2.4 .1 Temporal profiles of gas and sputtered species . . . . . . . . . 56

$2.4 .2 \quad$ Effects of spatial sampling on ion temporal profiles . . . . . . 56

2.4 .3 Effects of power and pressure on ion temporal profiles and intensity . . . . . . . . . . . . . . . . . 6 61

2.4 .3 .1 Power. . . . . . . . . . . . . . . 61

2.4 .3 .2 Pressure. . . . . . . . . . . . . . 61

2.4.4 Effects of pulse width and duty cycle with constant frequency on ion temporal profiles and ion intensity . . . . . . . . . . . . 67

2.4 .5 Doubly charged gas ions . . . . . . . . . . . . . 70

2.5 Conclusions . . . . . . . . . . . . . . . . . . . . . . . 74

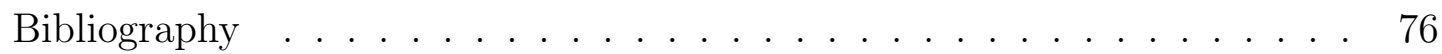

3 Application of Pulsed Glow Discharge Chemical Speciation: Chem$\begin{array}{lr}\text { ical Warfare Agent Metabolites } & \mathbf{7 9}\end{array}$

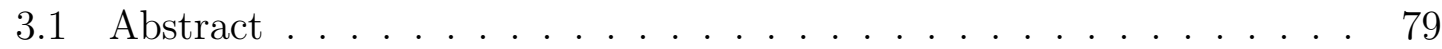

3.2 Introduction . . . . . . . . . . . . . . . . . . . . 80

3.3 Materials and methods . . . . . . . . . . . . . . . . . . . . . . . . . . 83

3.3 .1 Materials . . . . . . . . . . . . . . . . . 83

3.3 .2 Sample preparation . . . . . . . . . . . . . . . . 84

3.3 .3 Instrumentation . . . . . . . . . . . . . . . . . . . . . . . . . 84

3.4 Results and discussion $\ldots \ldots \ldots$. . . . . . . . . . . . . . . . . . . . . . . 89

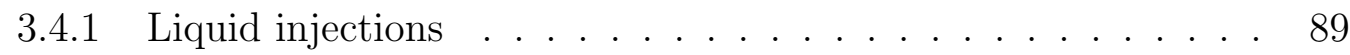

3.4 .2 Solid phase microextraction . . . . . . . . . . . . . . . . 89

3.5 Conclusions and future directions . . . . . . . . . . . . . . . . . . . . . . . . . . . . 95

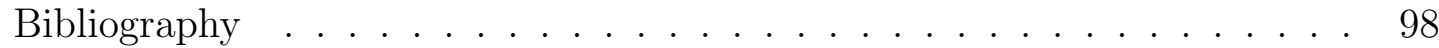

4 Application of Pulsed Glow Discharge Chemical Speciation: Explo$\begin{array}{ll}\text { sives } & 104\end{array}$

4.1 Abstract . . . . . . . . . . . . . . . . . . . . . . 104

4.2 Introduction . . . . . . . . . . . . . . . . . . . 105 
4.3 Materials and methods . . . . . . . . . . . . . . . . 106

4.3 .1 Materials . . . . . . . . . . . . . . . . . . . 106

4.3 .2 Instrumentation . . . . . . . . . . . . . . . . . . 107

4.3 .3 Data collection . . . . . . . . . . . . . . . . . . 110

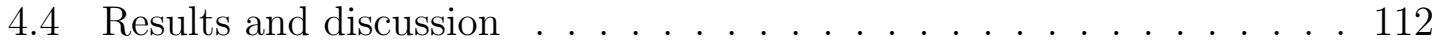

4.5 Conclusions and future directions $\ldots \ldots \ldots \ldots \ldots$

Bibliography . . . . . . . . . . . . . . . . 116

5 Measuring Intrinsic Molecular Properties of Microbial Particles for Attribution Purposes 118

5.1 Abstract . . . . . . . . . . . . . . . . . . . 118

5.2 Introduction . . . . . . . . . . . . . . . . . . . . . . . . . . . 119

5.3 Materials and methods . . . . . . . . . . . . . . . . . . . . 121

5.3 .1 Materials . . . . . . . . . . . . . . . . . . . . 121

5.3 .2 Pycnometer . . . . . . . . . . . . . . . . . . . . . . . . 121

5.3 .3 Particle size distribution analyzer $\ldots \ldots \ldots \ldots$

5.3 .4 Particle size fractionation . . . . . . . . . . . . . . . . . . . 122

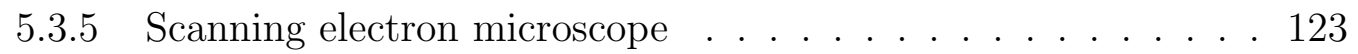

5.3 .6 Raman spectrometer . . . . . . . . . . . . . . . . . . . 123

5.4 Results and discussion $\ldots \ldots \ldots \ldots$

5.4 .1 Density $\ldots \ldots \ldots \ldots \ldots$

5.4 .2 Particle size distribution . . . . . . . . . . . . . . . . . . . 123

5.4.2.1 APS comparison with MOUDI cascade impactor . . 124

5.4 .3 Morphologic analysis of Bacillus globigii spores . . . . . . . 130

$5.4 .4 \quad$ Molecular composition $\ldots \ldots \ldots \ldots \ldots \ldots$

5.5 Conclusions and future directions $\ldots \ldots \ldots \ldots \ldots$

Bibliography . . . . . . . . . . . . . . . . . . . . . . . 139

\begin{tabular}{|l|l|l|l}
\hline Abbreviations and Acronyms & 144
\end{tabular} 


\section{List of Figures}

1.1 Mass spectrum of copper by glow discharge ionization. . . . . . . . . 4

1.2 Basic components of a mass spectrometer. . . . . . . . . . . . 5

$\begin{array}{lll}1.3 & \text { Schematic diagram of the different spatial regions in a glow discharge. } & 7\end{array}$

1.4 Sputter atomization process occurring in a glow discharge. . . . . . . 8

1.5 Temporal ion signal profiles: (A) voltage power supply, (B) sputtered analyte ions, (C) discharge gas ions. . . . . . . . . . . . . . . . 13

1.6 Principle of a time-of-flight mass spectrometer. . . . . . . . . . . . . . . 19

1.7 Microchannel plate detector. . . . . . . . . . . . . . . . . . . 23

1.8 Schematic diagram of a gas chromatograph. . . . . . . . . . . . . . . 26

1.9 SPME fiber and holder. . . . . . . . . . . . . . . . . . . . . . 28

1.10 Schematic diagram of a gas displacement pycnometer. . . . . . . . . . 28

1.11 Schematic diagram of a small scale powder disperser. . . . . . . . . . . 30

1.12 Schematic diagram of an aerodynamic particle sizer. . . . . . . . . . . . 32

1.13 Schematic diagram of a typical MOUDI stage showing its relation to stage above and stage below. . . . . . . . . . . . . . . 33

1.14 Schematic diagram of a scanning electron microscope. . . . . . . . . . 36

1.15 Energy state diagram showing the states involved in three different forms of scattering and their resulting spectrum. . . . . . . . . . . . . 38

2.1 Diagram of glow discharge ion source with time-of-flight mass spectrometer. ....................... 52

2.2 Sample mass spectra from different time regimes of the GD pulse. . . $\quad 55$

2.3 Pulsed voltage waveform and typical ion temporal profiles observed in a pulsed argon GD . . . . . . . . . . . . . . . 57

2.4 Effect of distance from cathode to ion exit orifice on ion intensity profiles. 59

2.5 Effect of instantaneous GD pulse power on ${ }^{63} \mathrm{Cu}^{+}$intensity profile. . . 62

2.6 Effect of discharge gas pressure on location of maximum ion intensity. 64

$2.7 \quad$ Effect of discharge gas pressure on the ion profile of ${ }^{63} \mathrm{Cu}^{+}$. . . . . . 66 
2.8 Effect of discharge gas pressure on arrival time of sputtered species. . 68

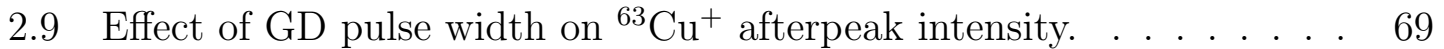

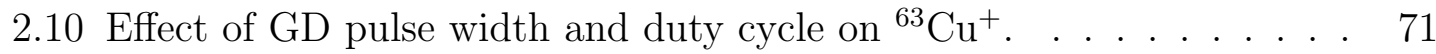

2.11 Effects of cathode distance and discharge gas pressure on the intensities of ${ }^{40} \mathrm{Ar}^{2+}$ and ${ }^{40} \mathrm{Ar}^{+}$at plasma initiation. . . . . . . . . . . 73

3.1 Reaction pathway for degradation of sarin to IMPA and MPA. . . . . 81

3.2 Derivatization of IMPA with MTBSTFA. . . . . . . . . . . . . . . . . 85

3.3 Schematic diagram of the GC-GD interface. . . . . . . . . . . . . . . 88

3.4 Mass spectrum of methanol and MTBSTFA sample blank and mass spectra of derivatized IMPA sample. . . . . . . . . . . . . . . . 92

$3.5 \quad$ Effect of discharge gas pressure on mass spectra of derivatized IMPA. 93

4.1 Gas chromatography glow discharge time-of-flight mass spectrometer. 108

$4.2 \quad$ Time gated acquisition of mass spectra from three different temporal regimes of a single glow discharge pulse. . . . . . . . . . . . . . . 111

$4.3 \quad$ Mass spectra of nitrobenzene produced using pulsed GDMS with timegated acquisition from three different temporal regimes. . . . . . . . . 113

4.4 Background-subtracted mass spectra of nitrobenzene produced using pulsed GDMS with time-gated acquisition from three different temporal regimes. . . . . . . . . . . . . . . . . . . . 114

5.1 Average APS number-weighted size distribution for BG spores. . . . . 125

5.2 Average mass-weighted size distributions for polystyrene DVB micro-

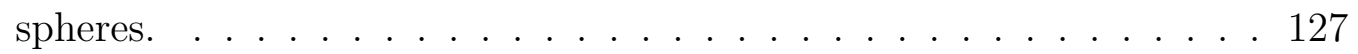

5.3 Average mass-weighted size distributions for aluminum oxide particles. 128

$5.4 \quad$ Average mass-weighted size distributions for aluminum silicate particles. 129

5.5 Average mass-weighted size distributions for BG spores. . . . . . . . . . 131

$5.6 \quad$ Scanning electron micrograph images of uncoated B. globigii spores. 133

5.7 Raman spectra of a polystyrene cup, polystyrene bulk powder in a glass vial, and polystyrene powder dispersed onto a MOUDI foil substrate. . . . . . . . . . . . . . . . . . . 135

5.8 Raman spectra of (a) blank foil substrates, (b) size-fractionated BG spore sample on foil substrates, and (c) bulk sample of BG spores. . . 136 


\section{List of Tables}

$1.1 \quad$ Ionization Potentials of Species Commonly Found in Glow Discharges. 9

2.1 Pulsed GD-TOFMS Operating Conditions . . . . . . . . . . . . . . . 54

3.1 GC/Pulsed GD-TOFMS Operating Conditions . . . . . . . . . . . . . 87

4.1 Examples of High Explosives . . . . . . . . . . . . . . . . . . . . 105

4.2 GC/Pulsed GD-TOFMS Operating Conditions . . . . . . . . . . . . . 109

5.1 Pycnometer measurements for Bacillus globigii particulate sample. . . 124 


\section{Chapter 1}

\section{Introduction}

If we knew what it was we were

doing, it would not be called

research, would it?

Albert Einstein

Analytical chemistry is the development and application of methods and their diverse techniques to separate, identify, or quantify the matter or analyte. To achieve these goals, a tremendous number of instrumental techniques have evolved allowing new possibilities to the modern day analytical chemist, notably hyphenated methods and non-destructive methods. Because a given sample can often be measured by several different methods, to determine the best, there are several criteria an analytical chemist must consider: sensitivity, robustness, linear dynamic range, accuracy, precision, speed, and cost, among others. An analytical chemist must not only understand the sample under examination, but also posses an understanding of the instrumental technique and be able to interpret the results obtained to be successful. This chapter introduces a variety of instruments and techniques that were used in subsequent chapters of this dissertation.

This dissertation opens with an introduction to the basic methodology and instrumentation used in this dissertation. This chapter is intended as an instrumentation primer, providing the reader with a general foundation for the study of subsequent chapters. The goal is not to provide a thorough technical explanation, but rather to provide a basic, yet accurate understanding of the instrumentation on which a more in-depth learning process can be based. A great deal of attention has been given 
to the basic principles of the glow discharge ionization source, which is crucial in understanding subsequent chapters.

\subsection{Mass Spectrometry}

To an analytical chemist, perhaps one of the most important analytical tools is mass spectrometry (MS). Analytical chemists are consistently drawn to mass spectrometry because of its sensitivity, low detection limits, ability to provide both qualitative and quantitative information, and wide application range.1 The instruments are applied to a variety of analytical problems, ranging from elemental isotope ratio determination to protein identification.

In the early 1900's, mass spectrometry was born at the hands of J. J. Thomson of the University of Cambridge. He constructed an instrument in which electrostatic and magnetic fields were used to separate ions by their mass-to-charge ratios and the spatial dispersion of the ions were detected on a fluorescent screen or photographic plate. ${ }^{2}$ His work revealed that not only were the obtained spectra characteristic of the discharge support gas, but also considerably less complex than the corresponding optical spectra. In 1913, Thomson found that neon consists of two isotopes, ${ }^{20} \mathrm{Ne}$ and ${ }^{22}$ Ne. Thomson's student, F. W. Aston, developed the first mass spectrometer in which ions were separated by mass-to-charge ratios and focused with velocity. ${ }^{3}$ In 1922, he was awarded the Nobel Prize for his discovery of stable isotopes and for his formulation of the whole-number rule.

Following the first studies of Thomson and Aston, tremendous technological improvements have led to the development of entirely new instruments and applications. The concept, however, is still the same; mass spectrometry is based on the motion of ions according to their mass-to-charge ratios. It is the determination of masses of atoms, molecules, or fragments of molecules of ions from individual species in a sample that makes MS an indispensable analytical method.

Different ions will have different mass-to-charge ratios $(m / z)$, that is the numerical value for the mass $(m)$ of the ion divided by its number of charges $(z)$. Mass spectrometers use these differences in $\mathrm{m} / \mathrm{z}$ ratios of ionized atoms or molecules to separate them in space or time. Mass spectrometers use electric or magnetic fields to apply a force on the ions. This force causes an acceleration that is mass-to-charge dependent; the lighter and more charged the atom or molecule, the faster its acceleration. 
Ions are generated by inducing either the loss or gain of a charge from a neutral species. For most mass spectrometry experiments, one electron is lost during ionization so $z$ is +1 and the $m / z$ value is equivalent to the relative molecular mass of the ion. Once formed, the ions are electrostatically directed into a mass analyzer where they are separated according to $m / z$ and finally detected. Ions separated in a mass spectrometer according to their mass-to-charge ratio are detected in proportion to their abundance. The result of ionization, separation, and detection is a mass spectrum - a graph that represents an ion's abundance as a function of its $\mathrm{m} / \mathrm{z}$ in increasing order, Figure 1.1. The interpretation of this information allows for the determination of the nature, composition, and even structure of the analyte.

During analysis by a mass spectrometer, the sample undergoes a number of successive processes, most importantly ionization, separation, and detection. To achieve this, mass spectrometers possess three primary components: an ion source (for ionization), a mass-selective analyzer (for separation), and an ion detector (for detection), as shown in Figure 1.2. There are a number of ionization techniques (electron ionization, chemical ionization, electrospray, etc.), a number of mass analyzers (magnetic-sector, quadrupole, ion trap, etc.), and several detectors that can be combined in various ways to comprise a mass spectrometer. In the following sections, a glow discharge ion source, time-of-flight mass analyzer, and microchannel plate detector are discussed.

\subsection{Ion Source: Glow Discharge}

Mass spectrometry pioneers, Thomson and Aston, used electrical discharges as ionization sources on some of the earliest mass spectrometers constructed. However, electrical discharges fell out of favor as newer and more novel ionization sources were designed. It wasn't until the early 1970's when electrical discharges, specifically the glow discharge, received renewed interest as ionization sources for mass spectrometry in the analysis of solids using both direct current and radio frequency discharges. ${ }^{4 / 5}$

The glow discharge is a relatively simple and inexpensive device, yet a powerful analytical tool with a variety of analytical uses spanning many disciplines including chemistry, physics, and materials science among others. The prime application of a glow discharge ionization source is the direct elemental analysis of inorganic solid samples ${ }^{6-9}$ and is well suited to the direct analysis and depth-profiling of conductive and non-conductive solid samples. However, the glow discharge ionization source has been successfully used in conjunction with liquid chromatography ${ }^{10}[12$ and gas 


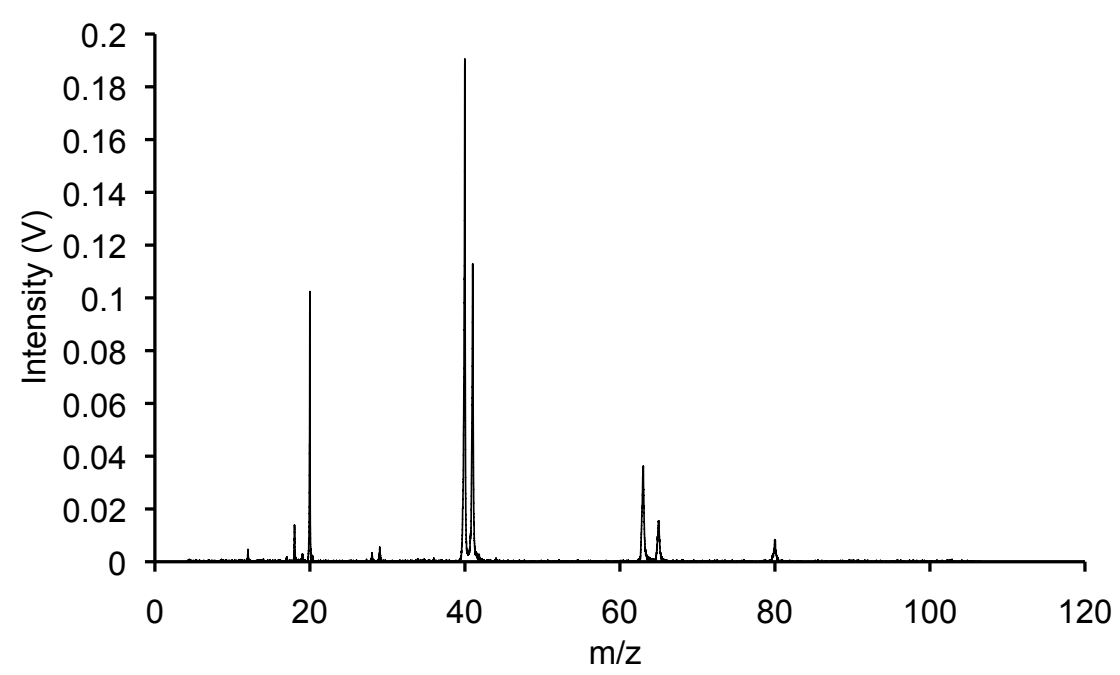

Figure 1.1: Mass spectrum of copper by glow discharge ionization.

chromatography ${ }^{13}$ separation techniques to expand its application.

A great deal of research and development has led to hundreds of publications on glow discharges. Three books in particular serve as invaluable references for learning the fundamental characteristics and applications of glow discharge plasmas ${ }^{17}[19$ in addition to various review articles. $20+26$

\subsubsection{Establishment of a Glow Discharge}

Glow discharges exist in our everyday lives in the form of neon advertising signs. fluorescent lamps, and plasma-screen televisions. We can visualize this phenomena, as the emission of light is one of the principal characteristics of discharges. In fact, the name, glow discharge, arises from the relatively bright central glow originating from excited gas atoms emitting their characteristic optical radiation.

A glow discharge (GD) is a plasma - a partially ionized gas consisting of electrons and positive ions, as well as a large number of neutral species. A GD is formed through the application of a potential difference (on the order of $1 \mathrm{kV}$ ) between two electrodes that are immersed in a low-pressure (0.1-10 Torr) inert gas environment. Through the use of a voltage supply, an electric field is established between the two 
Mass Spectrometer

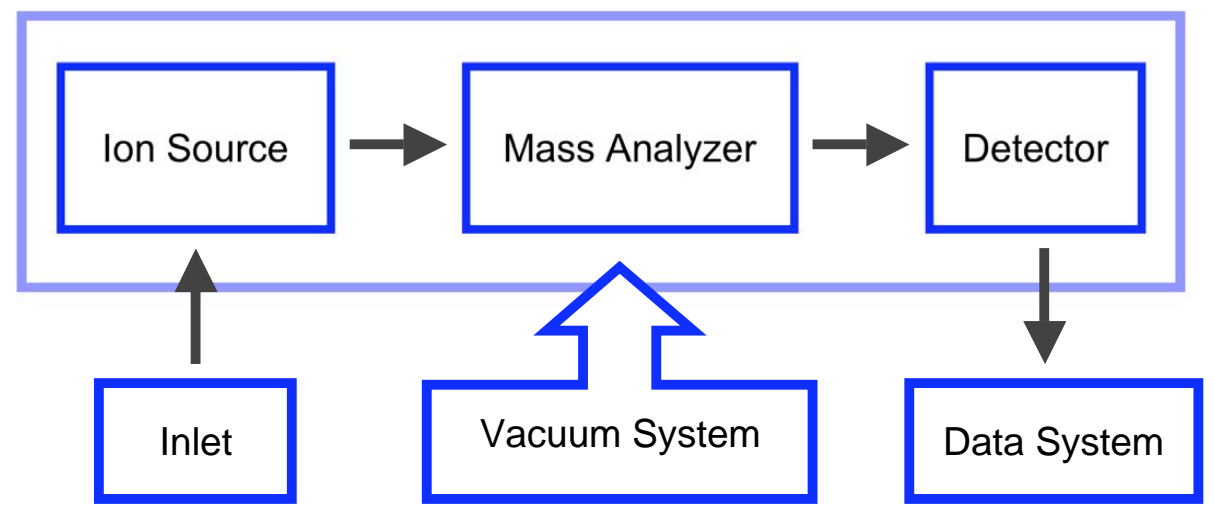

Figure 1.2: Basic components of a mass spectrometer.

electrodes such that one electrode is more negative than the other. The electrode with a relative lower, or negative, potential $(-)$ is the cathode and the electrode with a relative higher, or positive, potential $(+)$ is the anode. Because electrons have a negative charge, the potential difference that arises between the two electrodes causes electrons already in the electrical discharge, such as the random electrons produced by cosmic rays and radioactivity, to accelerate away from the cathode. During their travel, these primary electrons, accelerated by the electric field, occasionally interact with discharge gas atoms. A fraction of these collisions will produce excitation of the gaseous medium and a fraction will be of sufficient energy to remove an electron from a discharge gas atom, creating secondary electrons and positively charged ions, electron-ion pairs. The end result is partial ionization of the gas and the initiation of a glow discharge plasma.

It is the movement of these electron-ion pairs that permits current to flow. This flow of electric current through the ionized gas medium by the motion of free electrons and positive ions is termed "gas discharge." 27 The different types of gas discharges are classified based on the strength of current: Townsend discharge (microamperes), glow discharge (milliamperes), and arc discharge (amperes). 


\subsubsection{Spatial Regions of a Glow Discharge}

The glow discharge is made of several different spatial regions with quite different characteristics $^{28}$ named mainly for their luminosity. Beginning at the cathode and ending with the anode, the spatial regions are the Aston dark space, the cathode layer, cathode dark space, negative glow, Faraday dark space, positive column, anode dark space, and anode glow. For an in-depth look at the various regions of the glow discharge, the reader is directed to a book by A.M. Howatson titled An Introduction to Gas Discharges. 29

For analytical purposes, there exist three distinct regions to the glow discharge: the cathode dark space, the negative glow, and the Faraday dark space. Figure 1.3 illustrates these three different regions of a glow discharge. The cathode dark space is characterized by a region of low light intensity found immediately adjacent to the cathode surface. The electric field decreases linearly from the cathode to the edge of the negative glow owing to the space charge of positive ions ${ }^{28}$ in the negative glow. Almost all of the discharge potential is dropped across the cathode dark space. Lying just beyond the cathode dark space is the negative glow that appears as a luminous region surrounding the dark space and extending outward. The negative glow is quasi-equipotential ${ }^{30}$ consisting, on average, of equal numbers of positive ions and negative electrons in a sea of neutral atoms. For this reason it is not surprising that the overwhelming majority of collisional processes involve neutral gas species in the electronic ground state. 17

\subsubsection{Cathodic Sputtering}

The atomization of the sample results from the sputtering of the surface by gas-phase cations and fast gas-phase atoms. Following the production of the electron-ion pairs, these positively charged ions are accelerated by the potential difference towards the cathode. Before impact, these high energy ions can recombine with Auger electrons released from the cathode surface to form high energy neutrals that, in addition to the gas-phase cations, strike the cathode surface. ${ }^{32}$ Their kinetic energy is imparted to the sample's lattice structure through a collisional cascade process. If this energy is sufficient to overcome the binding energy of a surface atom, the atom will be released into the gas phase. Secondary species liberated from the cathode surface into the gas phase can include ionized and neutral atoms and molecules, small clusters of

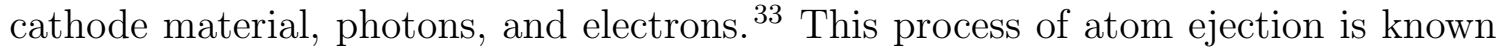
as sputtering (illustrated in Figure 1.4) and is the means of sample atomization 


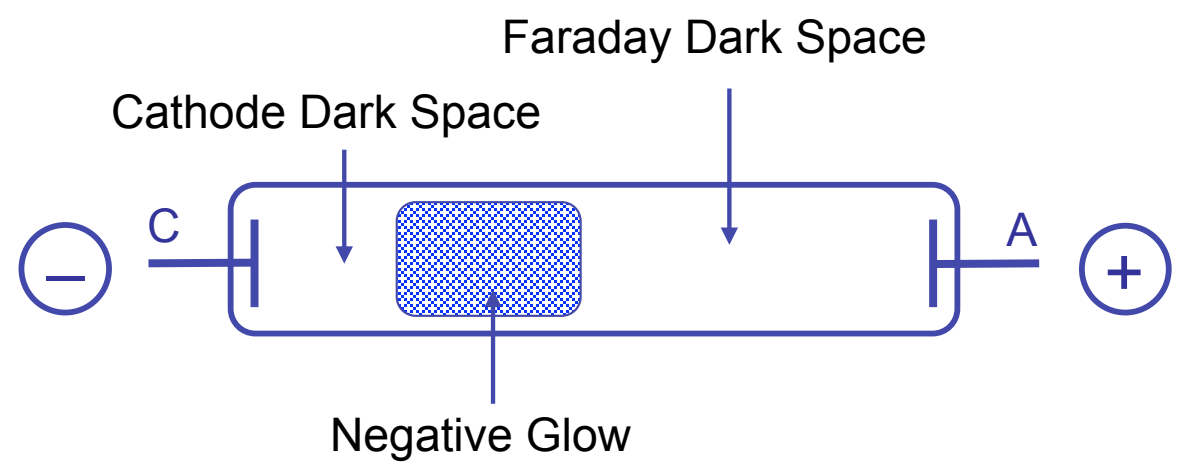

Figure 1.3: Schematic diagram of the different spatial regions in a glow discharge. Adapted from reference. 31

within glow discharge plasmas.

Species released from the cathode as positive ions are redeposited back onto the cathode surface by the potential gradient adjacent to the cathode. On the other hand, sputtered neutral atoms and molecules are free to diffuse into the negative glow region of the GD where they are subsequently excited and/or ionized through inelastic collisions, thus providing the basis for atomic emission and mass spectrometric analytical techniques. Secondary electrons, liberated from the cathode surface or as a by-product of ionization reactions, complete the cycle by accelerating away from the cathode resulting in a continuous flow of electrical current and a self-sustaining plasma.

Sputtering is a momentum transfer process involving the top 10 angstroms of material. ${ }^{34}$ This process is characterized by the sputter yield and rate. The sputter yield is defined as the number of atoms ejected per incident particle. The sputter yield is dependent on the complex function of (i) the target material's binding energy, (ii) the mass of the target atom, (iii) the mass of the sputtering gas, and (iv) the incident energy of the incoming particle. The sputter rate of a system describes the amount of cathode material removed per unit time. Fang and Marcus ${ }^{\sqrt{35}}$ studied in detail the dependency of the sputter rate on the discharge current and gas pressure in glow discharges. Plots revealed a linear relationship between sputter rate and discharge power, the product of current and voltage.

Routinely, solid samples to be analyzed by glow discharge ionization typically function as the cathode (at a negative potential), the ion source housing as the anode (at ground), and argon as the discharge gas. Argon is the inert gas of choice 


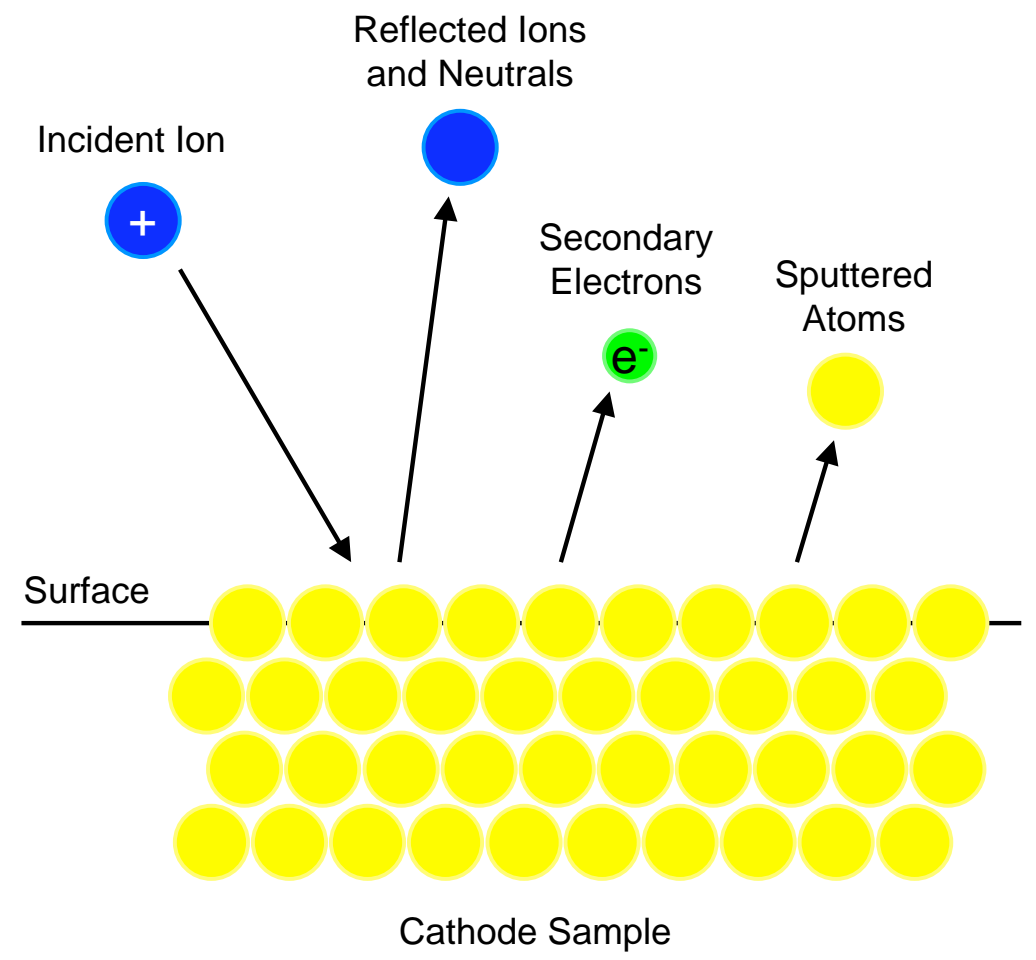

Figure 1.4: Sputter atomization process occurring in a glow discharge. Adapted from reference. 18 
for glow discharge plasmas for a number of reasons; while argon is not the largest or most massive (best for sputtering ${ }^{28}$ ) of the inert gases, it is the most economic choice for gas discharges as it is the most abundant in Earth's atmosphere. The nature of the glow discharge source affords the direct analysis of solid samples, thus minimizing any sample preparation required for analysis. An added benefit of glow discharge ionization is the separation of atomization and ionization into discrete steps. The mutually exclusive atomization and excitation/ionization processes help to minimize the elemental sensitivity and matrix effects that plague so many other techniques.

\subsubsection{Excitation and Ionization Processes}

The sputtered sample material diffuses into the discharge where interactions with electrons and excited gas atoms bring about excitation/ionization. Therefore, the plasma of the glow discharge can be used as an excitation source or an ionization source. In GD optical emission spectroscopy, the plasma is used as an excitation source. The GD generates photons of light by the excitation of electrons of a groundstate atom to a higher energy level. When the electrons "fall" back to ground state, wavelength-specific photons are emitted, which are characteristic of the element of interest. In GD mass spectrometry, the plasma is used as an ionization source. It is the ions, not the photons, generated by the plasma that are used in analysis.

Table 1.1: Ionization Potentials of Species Commonly Found in Glow Discharges.

\begin{tabular}{|c|c|c|}
\hline Species & 1st I.P. $(\mathrm{eV})$ & 2nd I.P. $(\mathrm{eV})$ \\
\hline $\mathrm{C}$ & 11.3 & 24.4 \\
\hline $\mathrm{N}$ & 14.5 & 29.6 \\
\hline $\mathrm{O}$ & 13.6 & 35.1 \\
\hline $\mathrm{Ar}$ & 15.8 & 27.6 \\
\hline $\mathrm{Ar}_{2}^{+}$ & $\begin{array}{l}15.2 \text { (direct ionization) } \\
14.6 \text { (associative ionization) })^{36}\end{array}$ & \\
\hline $\mathrm{Cu}$ & 7.7 & \\
\hline $\mathrm{H}_{2} \mathrm{O}$ & 12.6 & \\
\hline $\mathrm{N}_{2}$ & 15.6 & \\
\hline $\mathrm{CO}_{2}$ & 13.8 & \\
\hline
\end{tabular}

The inelastic collisions within the negative glow cause the emission of a number of visible spectral lines, which together give the discharge its characteristic luminous 
"glow." The glow results from the excitation and radiative relaxation of electrons from one discrete energy level to another. The color of the glow depends primarily on the gas used; an argon plasma emits a characteristic blue/purple glow.

Two collisional processes that occur within the dark space to a limited extent are electron ionization and asymmetric charge exchange. ${ }^{37}$ Electron ionization occurs when fast electrons collide with atoms or molecules resulting in the release of a bound electron and the formation of a positive ion. The generation of singly-charged ions by electron ionization and charge exchange is expressed in the following equations.

Electron Ionization

$$
e^{-}+X \rightarrow 2 e^{-}+X^{+} \quad\left(X=A r^{0}, C u^{0}, A r^{+}\right)
$$

Asymmetric Charge Exchange

$$
A r^{m+}+C u^{0} \rightarrow A r^{0}+C u^{+}
$$

However the most important region for ionization is the negative glow. The two main collisional processes which result in ionization that occur within the negative glow of the plasma are electron ionization and Penning ionization. ${ }^{19}$ Electrons are created at the cathode and are accelerated by a linearly decreasing electric field across the dark space. These energetic electrons cause ionization of the gas in the cathode fall and negative glow regions. The energy required to ionize argon, called the ionization potential, in the reaction $\mathrm{Ar} \rightarrow \mathrm{Ar}^{+}$, is $15.8 \mathrm{eV}$, and to liberate two electrons requires $27.6 \mathrm{eV}$. This gives an idea of the energy required to produce an electron-ion pair. A summary of ionization potentials of importance to an argon glow discharge can be found in Table 1.1 .

The formation of $\mathrm{Ar}_{2}^{+}$molecular ions is illustrated in the following equations.

Direct Ionization

$$
e^{-}+A r_{2} \longrightarrow A r_{2}^{+}+2 e^{-}
$$

Associative Ionization

$$
A r^{+}+A r \longrightarrow A r_{2}^{+}
$$

When a metastable atom collides with a neutral, a transfer of potential energy from the metastable to the neutral can occur and cause an electron to be ejected if the ionization energy of the neutral is below the energy of the excited metastable state of the gas atom. This is known as Penning ionization. With metastable po- 
tential energies of 11.55 and $11.72 \mathrm{eV}$, argon metastables are capable of ionizing most elements and molecules, while avoiding secondary ionization potentials and the ionization of atmospheric contaminants ${ }^{33}$ such as nitrogen and oxygen.

The processes leading to the formation of copper ions through Penning ionization are shown below where $\mathrm{Ar}^{m *}$ denotes a metastable argon atom and $\mathrm{Cu}^{*}$ an excited copper atom.

$$
e^{-}+A r^{+} \longrightarrow A r^{*} \longrightarrow A r^{m *}+h \nu
$$

Penning Ionization

$$
\begin{gathered}
A r^{m *}+C u^{0} \longrightarrow A r^{0}+C u^{+}+e^{-} \\
A r^{m *}+C u^{0} \longrightarrow C u^{*}+A r^{0} \\
C u^{*}+C u^{*} \longrightarrow C u^{+}+C u^{0}+e^{-}
\end{gathered}
$$

\subsubsection{Pulsing of the Glow Discharge}

The steady-state operation of the glow discharge discussed above produces a constant population of ions for analysis. Higher power discharges should result in larger ion signals and enhanced detection limits. However, steady-state discharges are power limited because operation at high powers can lead to overheating of the cathode, unwanted thermal emissions, and unstable discharges $\frac{38}{}$ In 1990, Klingler, Harrison, and co-workers ${ }^{38}$ reported their findings when pulsing the high voltage of an analytical glow discharge (rather than using a conventional steady-state dc voltage). Originally, pulsing of the discharge was done to increase the instantaneous power applied during the 'on' portion and cool the cathode during the 'off' portion of the pulse cycle. It was soon discovered that when the discharge power of a GD source is pulsed, or modulated, the technique not only benefits from higher instantaneous power, but from the temporal separation of ionization processes as well. Klingler et al. showed that rapid surges in ion intensities for both sputtered and contaminant gas species were observed but occur at different portions of the pulse period, as can be seen in Figure 1.5. Pulsing the glow discharge and using time-gated detection permits spectral discrimination of ionization mechanisms within the plasma. ${ }^{[9]}$ [1] $\mathrm{Pulsed}$ GDs are commonly used for the elemental and molecular analysis of solids, ${ }^{[742]}$ but 
have also found use in the analysis of organic vapors. $\frac{15}{15}$

\subsubsection{The Prepeak}

The gas-phase species are the only ions to be seen in the mass spectra as the discharge power ramps up. Their ion signals then increase until reaching a maximum around $18 \mu \mathrm{s}$ at what is referred to as the prepeak of the pulse cycle. This prepeak, lasting around $20 \mu \mathrm{s}$ is closely related to the surge in current that occurs at power initiation. This surge evidently enhances the efficiency of atomic excitation. Following the surge in intensity for those species already in the gas-phase, the ion signal for the sputtered species, copper, appears and grows steadily in intensity. This delay in appearances between gas-phase species and sputtered species is a result of the processes responsible for generating sputtered species: atomization via sputtering, diffusion into the negative glow, and subsequent ionization. ${ }^{43}$

\subsubsection{The Plateau}

Approximately $50 \mu \mathrm{s}$ into the pulse cycle, the signal intensity levels off and remains fairly constant until discharge termination, what is known as the plateau portion of the pulse cycle. The plateau portion represents an equilibration between ion formation and recombination processes and spectra from this time domain are similar to that of a steady-state glow discharge. Ionization mechanisms occurring within this temporal region include electron ionization, Penning ionization, and charge exchange. ${ }^{[19}$ During the plateau, electrons are continually released from the cathode and are also continually formed as by-products of ionizing collisions. Furthermore, the plateau is positioned during the on portion of the pulse allowing for the acceleration of electrons across the dark space entering the negative glow with fairly high velocities such that they are capable of electron ionization. Unlike the prepeak, during the plateau electrons have sufficient time to thermalize through inelastic collisions in the negative glow. Thermalized electrons may then recombine with argon ions to form metastable argon atoms which in turn are responsible for Penning ionization. Unlike electron and Penning ionization, in which ionization is possible for all elements having an ionization potential below the energy possessed by the electron or metastable argon, asymmetric charge exchange is a selective process between a rare gas ion and a metal atom. ${ }^{44}$ When the two collide an electron can be transferred from the atom to the ion only if an almost direct overlap of the energy levels for the rare gas ion ground (or metastable) state and the resultant metal ion exist. For this 


\section{POWER SUPPLY}
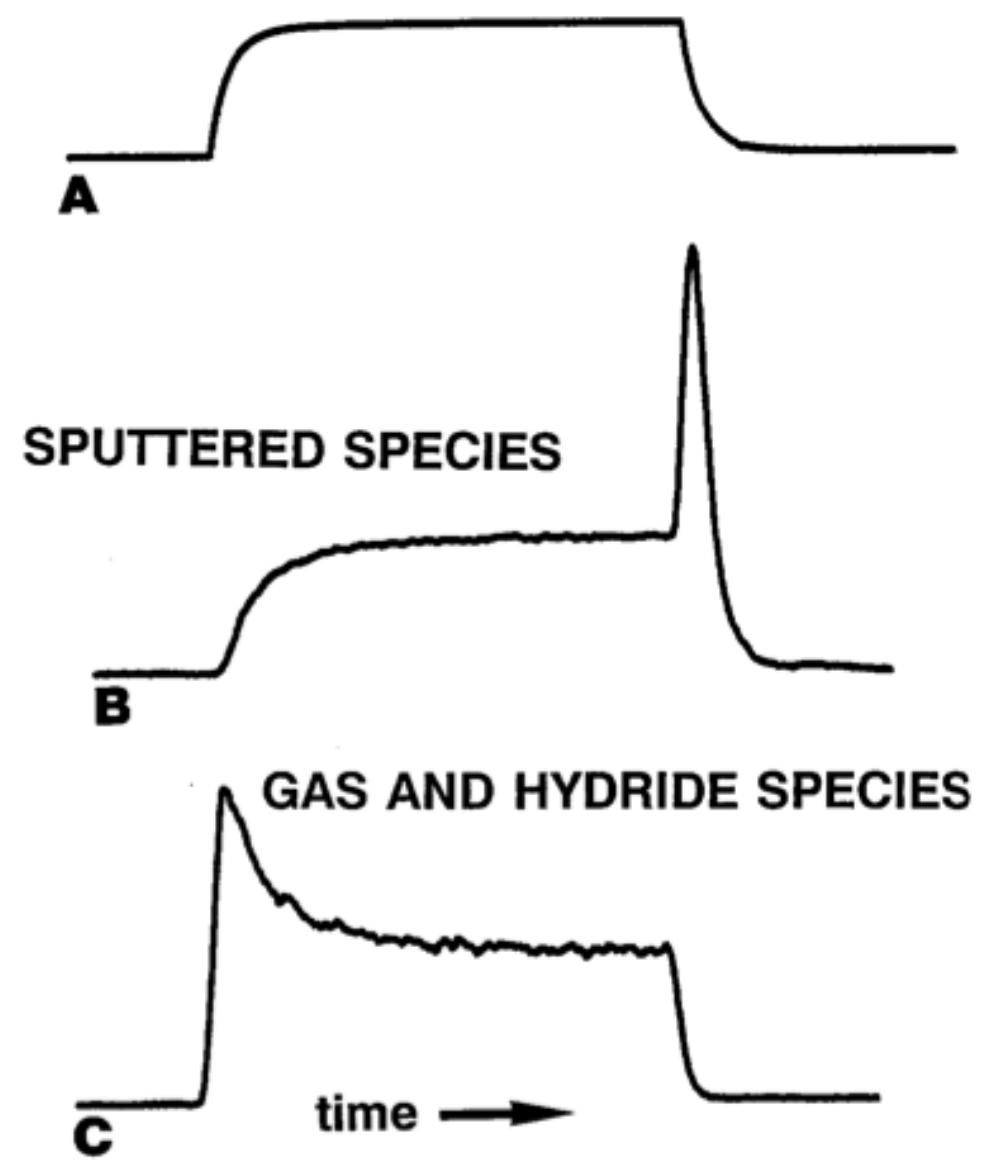

Figure 1.5: Temporal ion signal profiles: (A) voltage power supply, (B) sputtered analyte ions, (C) discharge gas ions. Reproduced from reference. $\underline{38}$ 
reason, ionization by positive ions is a less favorable process than the corresponding ones caused by electrons and metastable atoms. However, charge exchange has been shown to occur in copper-argon discharges, $\stackrel{45}{ }$ but only during the plateau portion of the pulse cycle ${ }^{\sqrt{46}}$ when a ground state copper atom and a metastable argon ion collide, producing an excited state copper ion. The number of charge exchange collisions relies heavily on the number density of the gaseous medium and the distance the ion must travel from the negative glow interface. 47

\subsubsection{The Afterpeak}

Following power termination, electron ionization halts and discharge gas (e.g. $\mathrm{Ar}^{2+}$, $\mathrm{Ar}^{+}, \mathrm{Ar}_{2}^{+}$) and contaminant (e.g. $\mathrm{H}_{2} \mathrm{O}, \mathrm{N}_{2}, \mathrm{CO}_{2}$ ) gas-phase species experience a sharp drop in intensity down to baseline, whereas sputtered species $\left(\right.$ e.g. $\left.\mathrm{Cu}^{+}\right)$behave quite differently. Sputtered species exhibit a rapid increase in intensity followed by a comparatively gradual decay to baseline intensities during what is referred to as the afterpeak portion of the pulse cycle.

It is at this point in the pulse cycle that fast electrons have lost much of their kinetic energy through inelastic collisions in the negative glow and can no longer be accelerated by the electric field making them incapable of ionizing any more of the gas-phase species. Yet these thermalized electrons, whose motion is diffusion controlled,,$\frac{47}{}$ can recombine with positive argon ions to form excited argon atoms. These atoms that remain in an excited state due to selection rules that forbid relaxation to the ground state are generally considered to be in a metastable state. $17 / 48$ These metastable species are important because their long lifetimes allow for reactive collisions to take place. The metastable potential energy levels for argon lie at 11.55 and $11.72 \mathrm{eV}, 49$ energies that are large enough to ionize the majority of sputtered metallic elements (IPs $\leq 11.72 \mathrm{eV}$ ) still remaining in the discharge chamber, but not sufficient for ionization of contaminant gas-phase species (IPs $>11.72 \mathrm{eV}$ ). In a metastable quenching study by Smith et. al. of steady state glow discharges, Penning ionization was found to account for approximately 40-80\% of the ionization of sputtered species in low pressure discharges of 0.4 to 1.2 Torr and discharge currents from 1 to $5 \mathrm{~mA} . \sqrt[50]{ }$ The rise and fall of the sputtered atom's afterpeak can

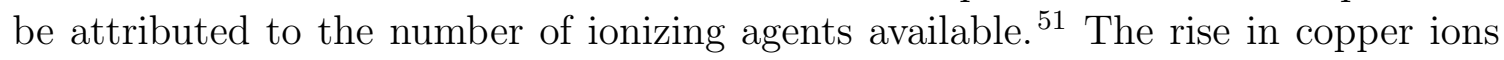
reflects an increase in metastable argon atoms available for ionizing collisions and the fall reflects a decrease in the number of metastable argon atoms as their lifetimes become exceeded. 52

Mass spectra captured during the afterpeak time regime represent an enhance- 
ment in the sputtered sample species with the near absence of interfering species. Therefore, introducing a time delay targeted for this region can provide cleaner mass spectra where the sample ions are the dominant signal. A prime example of this is a study by Lewis and co-workers in which the detection of ${ }^{40} \mathrm{Ca}^{+}$in the presence of ${ }^{40} \mathrm{Ar}^{+}$was accomplished using time-gated pulsed GD-MS. 53 Mass spectra were acquired during the afterpeak, during which time, temporal discrimination against electron-ionized interferences permitted the quantification of isobaric Penning-ionized species.

\subsubsection{Interface/Vacuum System}

In order to perform mass analysis on a sample, which is initially at atmospheric pressure (760 Torr), it must be introduced into the glow discharge chamber (0.110 Torr) in such a way that the vacuum inside the instrument remains relatively unchanged. The glow discharge ion sources used in this dissertation are equipped with a direct insertion probe (DIP) that enters the GD chamber through a vacuum interlock. The use of a DIP allows for the easy adjustment of the plasma relative to the ion exit orifice. The DIP is constructed of a stainless steel tube, 0.5 inch in diameter, with an insulating handle. An insulated copper wire runs the length of the tubing with one end attached to a MHV connector and the other end attached to a high pressure vacuum feed using silver solder. Both the connector and feed are welded to the tubing. In comparison to a DC DIP, the RF DIP requires additional insulation. The sample which serves as the cathode is attached to the end of the DIP, inserted through the sample introduction system, and into the discharge chamber. The discharge chamber is then filled with argon gas and all electrical components are turned on.

All mass spectrometers operate under a high vacuum, a pressure much below atmospheric, to allow ions to reach the detector without colliding with other gaseous atoms or molecules. And as such, introducing a sample from the glow discharge ionization source into a mass spectrometer requires the transfer of the sample from a region of low pressure, $0.1-10$ Torr, into a region of high vacuum, $10^{-5}-10^{-9}$ Torr, without compromising the latter. These large differences in pressure are controlled with the help of small orifices (that transmit the ion stream through an ion optics region and into the mass analyzer region) and a differential pumping scheme.

The GD time-of-flight mass spectrometers used in Chapters 3 and 4 each employ a series of pumping regions to reduce the effect of the required pressure drop. Once the ions are produced in the plasma, they pass through a hole in the back plate of the glow 
discharge chamber referred to as the ion exit orifice and enter the ion optics region (first vacuum stage), which is maintained at a reduced pressure with a mechanical roughing pump. This evacuates the discharge cell to a pressure of approximately $10^{-3}$ Torr in the absence of discharge gas. At the opposite end of the ion optics region, the ions encounter a second orifice called the skimmer cone - a conical surface with a small hole $(0.6-1.2 \mathrm{~mm})$ at the apex. The ion stream is extracted through the skimmer cone and enters a region at the rear face, the mass analyzer region (second vacuum stage), where the pressure is further reduced. This region, which includes the mass analyzer components as well as the detector, is evacuated using a turbomolecular pump backed by a mechanical pump. Once an initial vacuum of about $10^{-3}$ Torr is obtained by the mechanical pump, the turbomolecular pump is activated to obtain pressures at $10^{-6}-10^{-7}$ Torr, allowing the time-of-flight analysis of the ion stream.

The coupling of a glow discharge ion source and a time-of-flight mass analyzer is easily accomplished in an orthogonal arrangement. This instrument configuration generates GD ions in a pulse as usual, but instead of extracting them along the axis of the pulse, the repeller plate is placed so its electric field gradient is at a right angle to the beam of ions. Effectively a section of the ion beam is selected and pulsed into the mass spectrometer perpendicular to the original direction of motion. This eliminates the high initial axial velocity distribution of the GD pulse from the arrival times of the ions, thus improving mass accuracy and resolution. Time-of-flight mass spectrometers are ideally suited for pulsed ion sources, such as the pulsed GD source,, 54 as they provide near-simultaneous detection of all ions with each injection pulse. This trait allows for an excellent diagnostic tool for plasmas and is essential for the temporal characterization of millisecond GD pulses.

\subsection{Mass Analyzer: Time-of-Flight Mass Spec- trometer}

Perhaps the simplest type of mass spectrometer is the time-of-flight (TOF) mass spectrometer. The TOF consists of a short extraction region, a drift region, and a detector. The basic principle of a TOF mass spectrometer is very straightforward; a packet of ions accelerated to the same kinetic energy having a distribution of $m / z$ ratios will have a corresponding distribution of velocities and arrive at the detector at different times. Important components of a TOF mass spectrometer as well as an illustration of ions traversing the flight tube are shown in Figure 1.6. 
In the beginning, TOF mass spectrometers were regarded as low-resolution instruments and thus, were not commonly used. For near perfect resolution, ions, under the influence of an external electric field, should begin their acceleration with the same (or zero) kinetic energy, at the same time, and from the same spatial plane perpendicular to the acceleration vector. In reality, variations in initial conditions, such as time of ion formation, initial location in the extraction field, and initial kinetic energy (before acceleration), create differences in flight times for ions with the same $\mathrm{m} / \mathrm{z}$ giving rise to losses in resolution. $\frac{55}{5}$ Methods for correcting these distributions are referred to as time, spatial, and energy focusing. Despite their simple concept, TOF mass spectrometers have undergone some remarkable developments to greatly improve mass resolution and permit the coupling to virtually any ionization source.

In 1955, Wiley and McLaren ${ }^{[56}$ published a TOF mass spectrometer design with improved resolution that later became the first commercially successful instrument. Pulsed ionization sources required pulse durations sufficient to produce a large number of ions, unfortunately, this limited resolution due to distributions in the time of ion formation. To address this issue, Wiley and McLaren achieved time focusing by pulsing the extraction voltage so that ions that were not formed at the same time were all accelerated at the same time. To address the effects on resolution of the initial spatial distributions of ions with the same $m / z$, Wiley and McLaren proposed a TOF mass spectrometer with a two-stage ion extraction/acceleration region. The first stage provided a low-voltage extraction field to provide additional energy to ions nearer to the back of the source to 'catch up' with ions formed near the source exit. The second stage accelerated all of the ions to their final kinetic energy. Thus, spatial focusing was achieved with this two-stage source, which moved the spatial focus plane down the flight tube toward the location of the detector. To correct for initial kinetic energy distributions, the Wiley and McLaren design used a delay between the formation of the ions and the extraction pulse, a technique they referred to as time-lag energy focusing. Ions formed by a pulsed ion source were allowed to separate according to their kinetic energy in a field-free region in the source for $10^{-7}$ to $10^{-6} \mathrm{~s},{ }^{1]}$ before a pulsed voltage allowed for ion extraction. During the time delay, ions drifted in the source according to their initial velocities, so that those with higher velocities traveling in the direction of the mass analyzer would gain less energy from the extraction field when that field was pulsed. In this way, they compensated for the kinetic energy spread of the initial ion packet. Unfortunately, this correction using time-lag energy focusing is mass-dependent, i.e. shorter time delays focus lighter ions. ${ }^{[57}$ Thus, enhanced resolving power was attainable only over a limited mass range. Nonetheless, the design by Wiley and McLaren demonstrated time, spatial, and energy focusing by the extraction pulse rise time, the amplitude of the 
pulse, and the time delay between ion formation and extraction, respectively.

In 1964, at a time when electronic technologies supporting TOF were limited, O'Halloran et al. ${ }^{58}$ constructed a TOF with an orthogonal configuration yet limited resolving power. Unfortunately, this early orthogonal TOF work was not published in the general literature and was instead relegated to a technical report. ${ }^{59}$ In 1989 , Dawson and Guilhaus ${ }^{60}$ re-introduced an orthogonal acceleration TOF as a new way to couple TOF with continuous ion sources. The instrument included an electron ionization source and a linear TOF mass analyzer. The orthogonal orientation of the ion source with respect to the flight tube reduced the dispersion in kinetic energy in the direction of the flight tube and increased resolution. Their work was influential in the development of commercial orthogonal TOFMS instruments.

\subsubsection{Time-of-Flight Fundamentals}

When an ion is placed within an electric field, it will be forced in the direction opposite to its polarity. For the analysis of positive ions in a TOF mass spectrometer, this positive electrode is called the repeller plate and the relatively negative electrode to which the ions accelerate toward is called the extraction grid. To be useful for analysis, the ions must all be ejected from the ion source at the same starting time. For a plasma ion source, ions enter the TOF while the repeller plate and extraction grid are at nearly the same potential. The voltage of the repeller plate is then rapidly pulsed (typically 1-2 $\mathrm{ss}$ ), resulting in a small group of ions being ejected through the extraction grid at the same time. After passing through the extraction grid, the group of ions enter a much stronger potential field, about ten times that of the field they just left. An accelerating grid held at a high negative potential creates this strong field and causes the group of ions to rapidly accelerate into the flight tube at constant velocity. Steering plates are used to direct the ions in a straight line, along the flight tube, to the detector at the other end. In order to create a field free drift region, the flight tube is bounded by an acceleration grid and a second grid placed just before the detector, both of which are at the same potential. The accelerated ions drift with mass-to-charge dependent velocities and separate into groups during their flight toward the detector. Ion flight times generally fall in the range of 10 to $200 \mu \mathrm{s} . \frac{55}{}$ Ions having the smallest $\mathrm{m} / z$ arrive at the detector first followed by ions of successively larger $\mathrm{m} / \mathrm{z}$ ratios. The time of arrival of the ions at the detector and their abundance is recorded. Time is converted to $\mathrm{m} / z$ to produce a mass spectrum.

For further information on TOF mass spectrometers, the reader is referred to an excellent book by Robert J. Cotter titled Time-of-Flight Mass Spectrometry. ${ }^{55}$ 

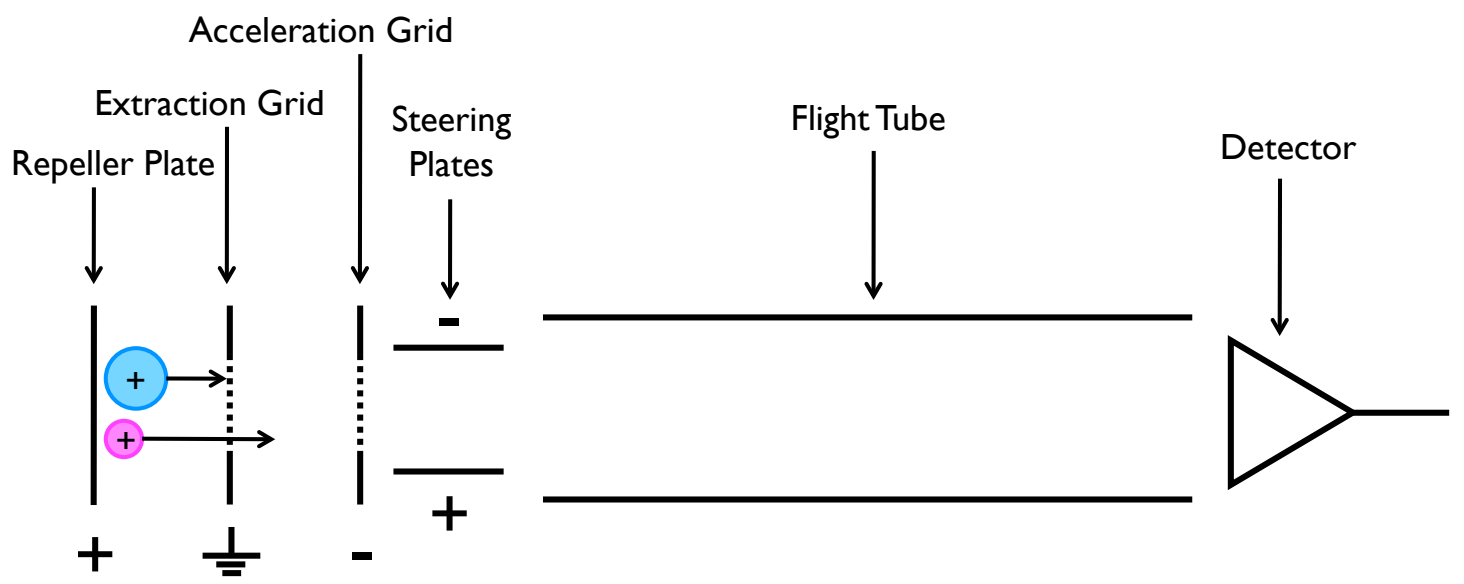

(a)
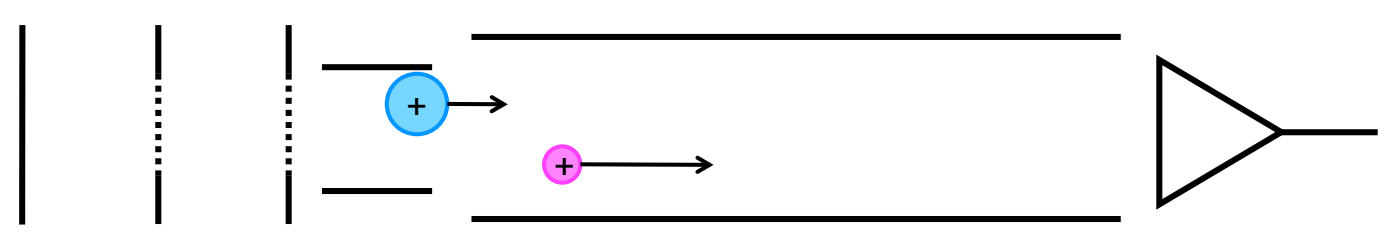

(b)

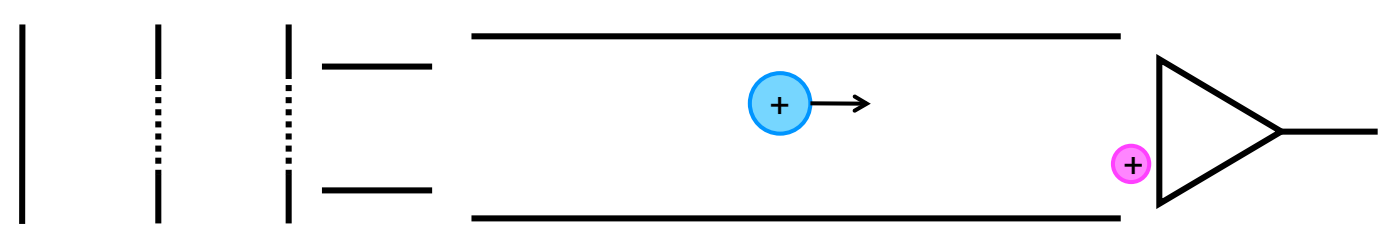

(c)

Figure 1.6: Principle of a time-of-flight mass spectrometer. (a) A pulse of ions is accelerated out of the source and (b) into the drift region where they are separated according to $\mathrm{m} / \mathrm{z}$. (c) Ions with the smallest $\mathrm{m} / \mathrm{z}$ (fastest moving) arrive first at the detector, followed by ions of sequentially increasing $\mathrm{m} / \mathrm{z}$. 


\subsubsection{The Time-of-Flight Equation}

In order to be useful for chemical analysis, the data output from mass spectrometers needs to be displayed in a mass spectrum: a plot of abundance vs. $m / z$. Let's look at some equations that help us convert an ion's time-of-flight to $m / z$.

In time-of-flight mass spectrometers, ions are accelerated by an electric field to the same final kinetic energy. The kinetic energy $(K E)$ of the ion is a function of mass $(m)$ and velocity $(v)$ and equals the product of the number of charges on an ion $(z)$, the charge on an electron $(e)$, and the electric field ( $E$ volts):

$$
K E=\frac{m v^{2}}{2}=z e E
$$

From this equation, one can deduce that the final velocity $(v)$, reached on acceleration of the ion to a common $\mathrm{KE}$, is inversely proportional to the square root of $m / z$. Rearranging Equation 1.9 for $v$ gives us the velocity at which an ion crosses the drift region:

$$
v=\left(\frac{2 z e E}{m}\right)^{1 / 2}
$$

The time $(t)$ taken for an ion to traverse the drift tube is equal to the distance $(L)$ from ion source to the detector divided by velocity $(v)$. Substituting Equation 1.10 for $v$ and solving for $t$ gives the ion's flight time:

$$
t=\frac{L}{v}=\left(\frac{m}{2 z e E}\right)^{1 / 2} L
$$

Thus, the time-of-flight of each ion is a measurement of the ion's velocity from which the $m / z$ ratio can be determined from the rearrangement of Equation 1.11 .

$$
\frac{m}{z}=\left(\frac{2 e E}{L^{2}}\right) t^{2}
$$

In Equation 1.12, $e$ is a universal constant, $E$ is held constant in the instrument, and $L$ is fixed. Thus, the $m / z$ ratio is proportional to the square of the flight time $(t)$ of an ion: 


$$
\frac{m}{z}=t^{2} \times \text { constant }
$$

\subsubsection{Calibrating the Mass Spectrum}

All mass spectra require some form of calibration equation to relate the physical property that is being measured, such as an ion's time-of-flight, to the measured mass. Using Equation 1.11, distance and kinetic energy are assumed to be constant and replaced with the constant $a$. Any time offsets are taken into account with the constant $b$.

$$
t=a m^{1 / 2}+b
$$

Using Equation 1.14 to calibrate a time-of-flight mass spectrum requires two standards. By measuring the flight times $(t)$ of two known masses $(m)$, the constants $a$ and $b$ in the above equation can be solved and the mass of unknown ions can then be derived.

\subsubsection{Resolution}

The ability of a mass spectrometer to separate two masses is described by the resolution, $R$, which is defined as:

$$
R=\frac{m}{\Delta m}
$$

where $m$ is the ion mass and $\Delta m$ is the full peak width measured at half of the peak maximum (FWHM). Alternatively, for a time-of-flight mass spectrometer, the resolution can also be calculated by:

$$
R=\frac{t}{2 \Delta t}
$$

where $t$ is the ion's flight time and $\Delta t$ is the peak width at half-maximum on the time scale. The attainable resolution of a TOF mass spectrometer is related to the duration of the ionization/repeller pulse, to the initial energy distribution of the ions

and to the precision with which the time of flight can be measured. ${ }^{61}$ High resolving power is normally associated with lower sensitivity due to discrimination of ions with 
broader energy distribution.

\subsection{Detector: Microchannel Plate Detector}

A detection system most often used with time-of-flight instruments is the microchannel plate (MCP) detector, Figure 1.7. TOF MS is typically operated in conjunction with MCPs, because this detector is characterized by its excellent time response designed to handle the fast ion pulses provided by the TOF mass spectrometer.

Measuring the current at the detector of a time-of-flight mass analyzer yields a time-dependent signal that can be correlated to the $m / z$ using Equation 1.11 . To accomplish this, MCPs convert incoming ions into electrons which are then amplified in a cascade by many orders of magnitude, i.e. they are compact electron multipliers of high gain. MCPs are fabricated from very thin glass plates that host a dense array $(\sim 10,000,000)$ of tiny tubes (microchannels) aligned parallel to one another and enter the plate at a small angle $\left(\sim 10^{\circ}\right.$ to the surface normal $\left.{ }^{62}\right)$. Both faces of the plate, as well as that of the channels, are coated with metal to provide parallel electrical connections (electrodes) to all channels. In a vacuum, under the presence of a strong electric field (typically $1 \mathrm{kV}$ ), each microchannel ( 10 $\mathrm{\mu m}$ diameter) acts as an independent, continuous-dynode electron multiplier. An ion that enters a microchannel through the front face of the plate (at the most negative potential) will strike the wall of the channel liberating one or more electrons, which will, in turn, start a cascade of electrons through the channel, liberating further electrons with each wall collision. After these electrons exit the plate at the back face (at the most positive potential), they are collected by an anode positioned parallel to the MCP. The overall result is a conversion of ions to electron current and an amplification of the original signal by several orders of magnitude (routinely $10^{4}$, but as much as $10^{6}-10^{7}$ electrons for every ion). The amplification of weak ionic current depends on the electric field strength, the ratio of length to diameter of the individual channels (typically 40:1 or 60:1), and the geometry of the microchannel plate.

Two or more MCPs can be arranged in a stack orientation to further amplify the signal, thus increasing the system's performance. A typical TOF MS has two microchannel plates, what is referred to as the chevron configuration, because their angled channels are orientated $90^{\circ}$ to each other in such a way that they produce a chevron ( $\mathrm{v}$-like) shape. Electrons exiting the first plate start the cascade of electrons in the next plate. Electrons exiting the last plate are collected by an anode, the electron current of which is converted to a voltage and amplified to the detector output. 


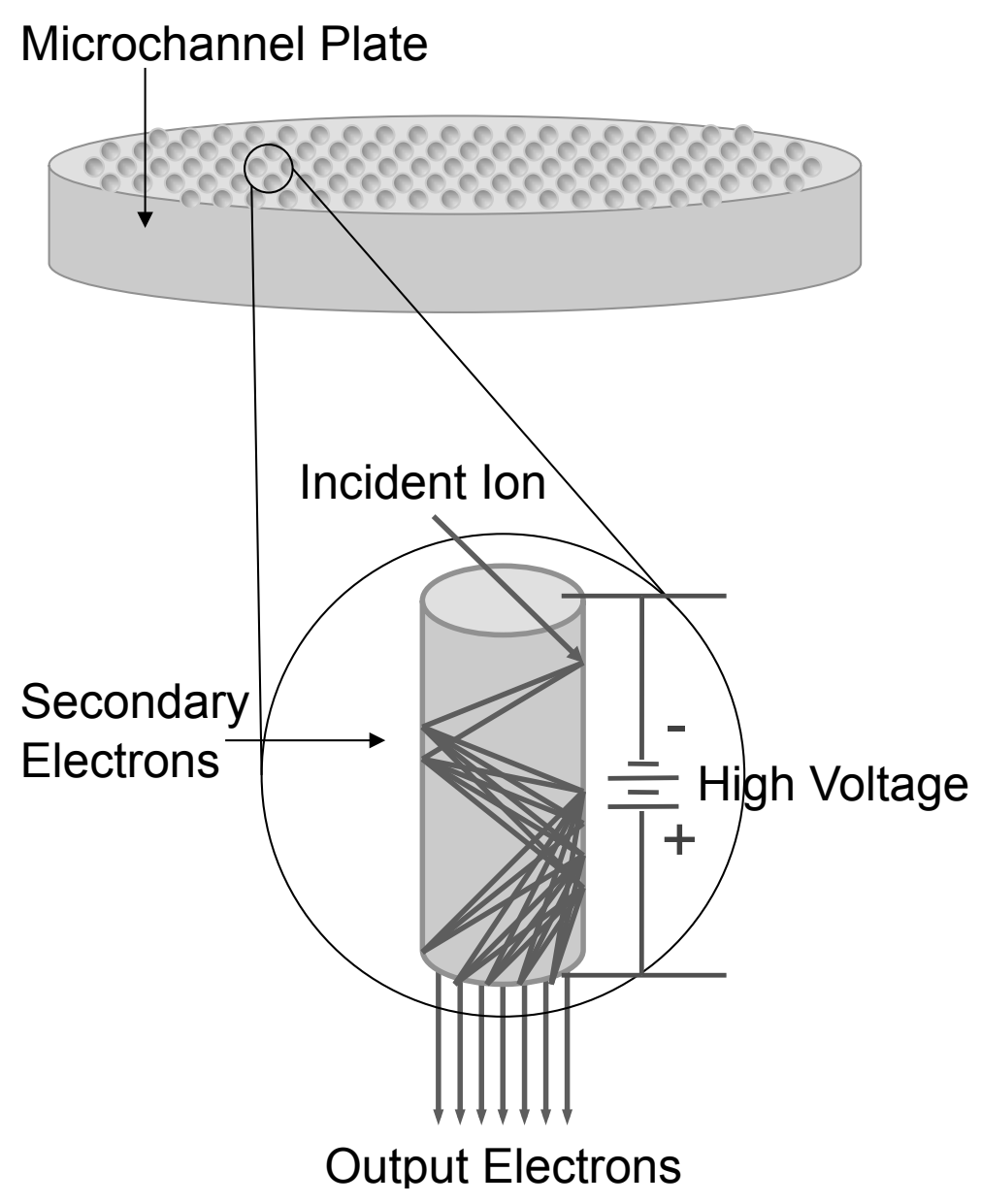

Figure 1.7: Microchannel plate detector. 
These stacked MCP assemblies provide significantly more gain at a given voltage than traditional straight channel MCPs. However, after the cascade of electrons, the microchannel takes time to recover before it can detect another signal, what is referred to as saturation, thus limiting the gain of multiple plate assemblies.

\subsection{Gas Chromatograph}

When mass spectrometry is combined with chromatography for the separation of compounds, MS can be used to study mixtures and samples containing trace amounts of analyte. The coupling of these techniques is an invaluable tool in the analytical chemists's arsenal.

Chromatography is the process by which the individual components of a mixture can be separated. The principle behind chromatography is based on the concentration equilibrium (i.e. distribution) of the compounds of interest between two phases: the stationary phase, which may be a solid, liquid, or gel and the mobile phase, which may be a liquid, gas, or supercritical fluid. The stationary phase is usually immobilized to the inner wall of a column and the mobile phase transports the components through the column. Components with different affinities for each phase will differ in their rates of migration through the column, leading to their separation. The components having the greater interaction with the stationary phase are retarded to a greater extent and consequently take longer to move through the column than those with a smaller interaction. Once the components have been separated, they are eluted from the column one after another and quantified by a detector and/or collected for further analysis at the column exit. The detector continually registers the variation of an electrical signal as a function of time. This detector signal leads to a chromatogram that indicates the variation of the composition of the eluting phase with respect to time.

Gas chromatography (GC) separates components of a mixture based on differences in vapor pressure and the interactions with a stationary phase and a gaseous mobile phase. GC was first suggested as an analytical method in 1941 by two British chemists, Martin and Synge. $\frac{63}{1 n}$ a paper on liquid chromatography, Martin and Synge theorized that the liquid phase used in liquid chromatography could be replaced by a suitable gas increasing the speed of the equilibrium processes involved 
in a chromatographic process:

"Very refined separations of volatile substances should therefore be possible in a column in which permanent gas is made to flow over gel impregnated with a-non-volatile solvent [...]"

A decade later, this concept became reality when James and Martin ${ }^{64}$ published a paper describing the first gas chromatograph, revolutionizing the field of chromatography. They reported the separation of volatile fatty acids by gas-liquid partition chromatography with nitrogen gas as the mobile phase and silicon oil/stearic acid supported on diatomaceous earth as the stationary phase. Early GC was carried out on straight, packed columns, typically $1-5 \mathrm{~m}$ long and $1-5 \mathrm{~mm}$ i.d. $\frac{65}{\mathrm{~A}} \mathrm{~A}$ high resistance to gas flow limited the length, and consequently the resolution, of packed columns. An increase in resolution was achieved with the invention of capillary columns. In 1957, capillary columns were introduced by Golay, ${ }^{[6]}$ who laid out the theory of operation and in $1958^{67}$ demonstrated its use. In comparison to packed columns, capillary columns have the advantages of increased separation efficiency and decreased analysis time. The very first capillary column investigated by Golay was an uncoated PTFE tube, however, he soon switched to glass and then to stainless steel tubes. ${ }^{68}$ Metal, plastic, and glass tubes were replaced with the invention of fusedsilica capillary columns. In 1979, Dandeneau and Zerenner ${ }^{69}$ introduced fused-silica capillary columns for GC that have the advantages of being highly flexible, durable, and chemically inert. Today, fused-silica, open-tubular, capillary columns are used almost universally in GC.

$\mathrm{GC}$ is a highly sensitive and selective separation technique, capable of resolving hundreds of components in a short time with part-per-million or better sensitivity. GC, however, is limited to separating and analyzing compounds that can be vaporized without decomposition. In the schematic gas chromatograph in Figure 1.8, volatile liquid or gaseous sample is injected through a septum into the heated injection port of a gas chromatograph, in which it rapidly vaporizes. The sample mixes with a stream of unreactive carrier gas (mobile phase), such as $\mathrm{He}, \mathrm{N}_{2}$, or $\mathrm{H}_{2}$, and is swept through a column, a coiled tube coated with a nonvolatile liquid or solid sorbant (stationary phase). The column is situated in a variable temperature oven and is where the analytes separate. Once the sample exits the column, it flows through a detector before exiting to the atmosphere. 


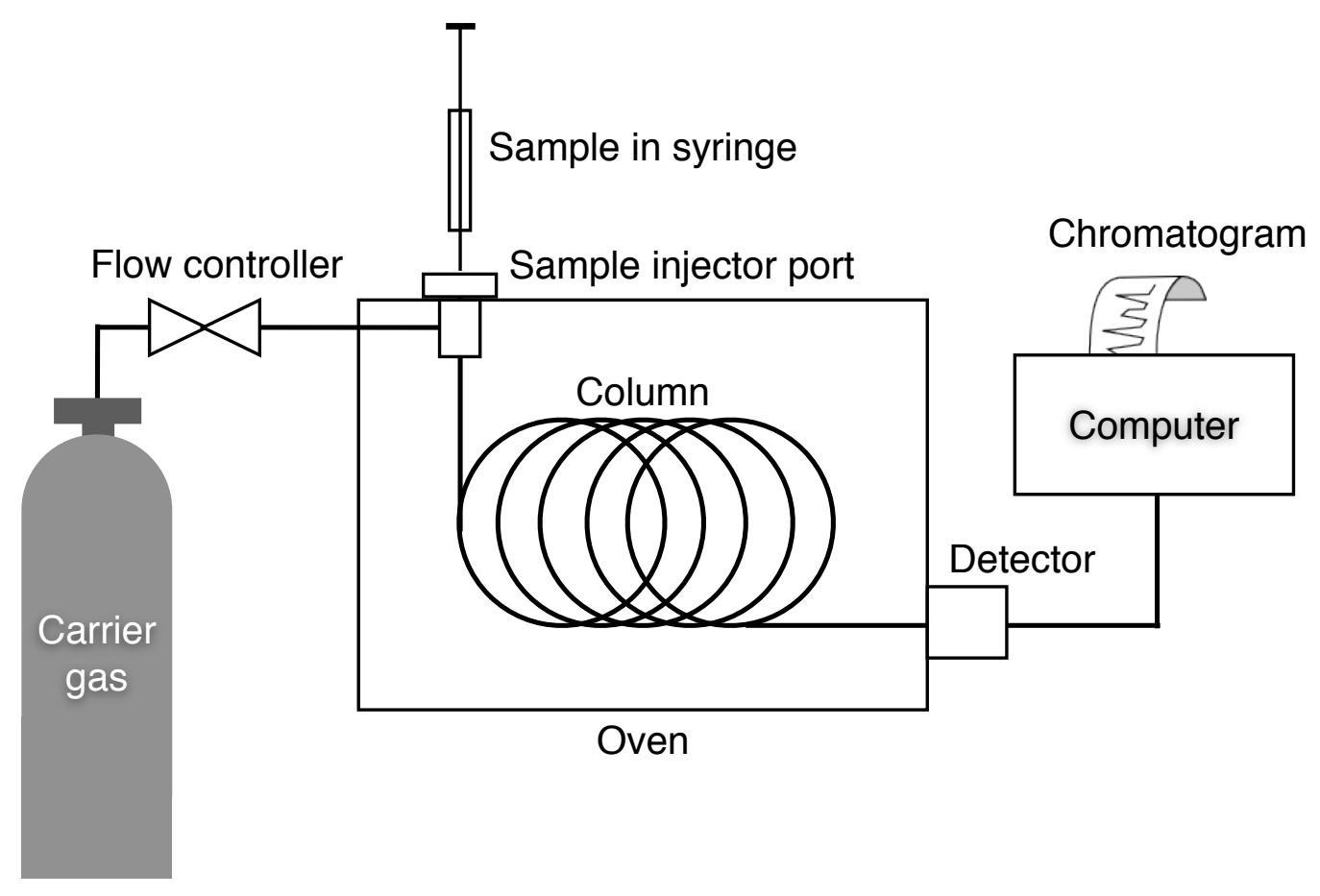

Figure 1.8: Schematic diagram of a gas chromatograph.

\subsection{Solid Phase Microextraction}

When applied to "real world" samples, the resolution and sensitivity of many analytical instruments (such as gas chromatography) are often limited by sample matrix effects. In such cases, a sample preparation step is necessary to first isolate the analyte from a sample matrix thus aiding in the analyte's separation and detection. One such sample preparation technique, solid phase microextraction (SPME), 70 -75 is capable of increasing analyte concentrations and detector response (useful in the analysis of dilute solutions) while reducing matrix effects that can otherwise interfere with separation and detection (useful in the analysis of complex matrices). The advantages of SPME over other sample preparation techniques are its low cost and simplicity.

SPME was invented by Janusz Pawliszyn of the University of Waterloo in the early $1990 s^{\frac{76177}{}}$ and has since revolutionized the way that samples are collected and extracted. SPME is a solvent-free sample preparation technique that uses a small 
fused-silica fiber coated with a layer of adsorbing polymer material. The fiber is housed within a syringe-like device that can expose and retract the fiber, Figure 1.9. When immersed in the sample, the SPME fiber directly adsorbs solutes from the sample matrix into the special coating. During extraction, the SPME fiber gradually reaches an equilibrium level with its surroundings, whether gas or liquid. In most cases equilibrium is reached within a few minutes. After extraction, the SPME fiber can be transferred and inserted directly into the injection port of a GC (or HPLC) system, where the analytes are thermally desorbed and subsequently separated and identified.

\subsection{Pycnometer}

The pycnometer is a non-destructive device that provides the density of small samples. In reality, it measures the sample volume only and incorporates the sample mass (from an external measurement) into its software to calculate the sample density. The AccuPyc 1330 Pycnometer from Micromeritics, Figure 1.10 is a gas expansion pycnometer that determines volume by measuring the pressure change (the displacement) of helium gas in a calibrated volume.

During the calibration process, the volumes of the instrument sample and expansion chambers are determined using a traceable standard volume. The sample is sealed in the sample chamber of known volume, Helium gas is admitted, and then expanded into the expansion chamber. The pressures observed upon filling the sample chamber (cell volume) and then discharging it into a second empty chamber (expansion volume) allow computation of the sample solid phase volume.

$$
V_{s}=V_{c}+\frac{V_{r}}{1-\frac{P_{1}}{P_{2}}}
$$

Dividing this sample solid phase volume into the entered sample weight, the pycnometer automatically derives the sample density:

$$
\text { density }=\frac{\text { mass }}{\text { volume }}
$$

This technique works well with powder samples as the small and inert helium gas molecules that the pycnometer uses rapidly fill the pores of the sample and only the truly solid phase of the sample displaces the gas. 


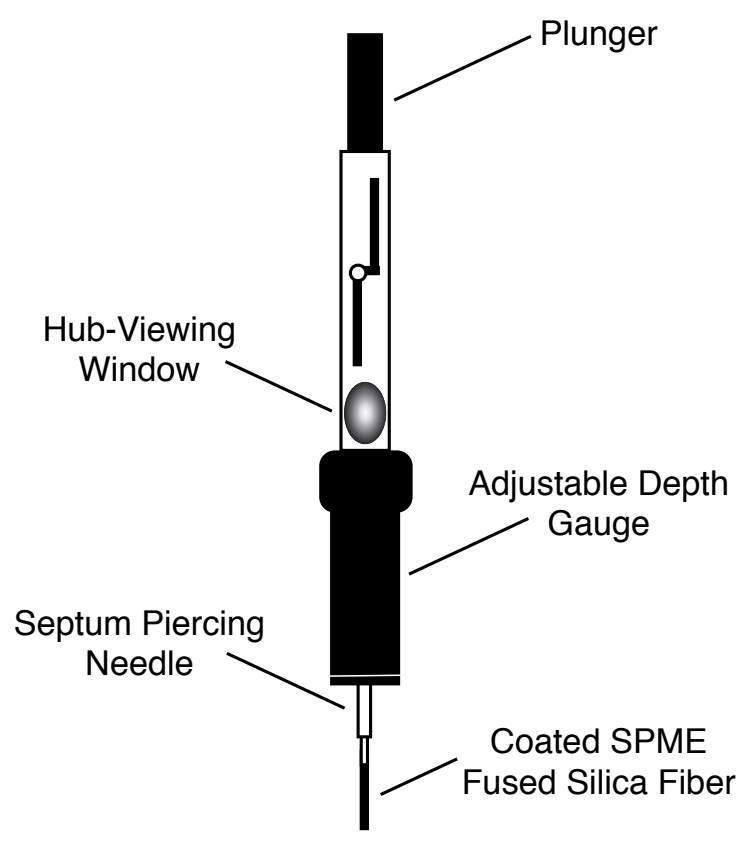

Figure 1.9: SPME fiber and holder. Adapted from Supelco. $\frac{78}{78}$

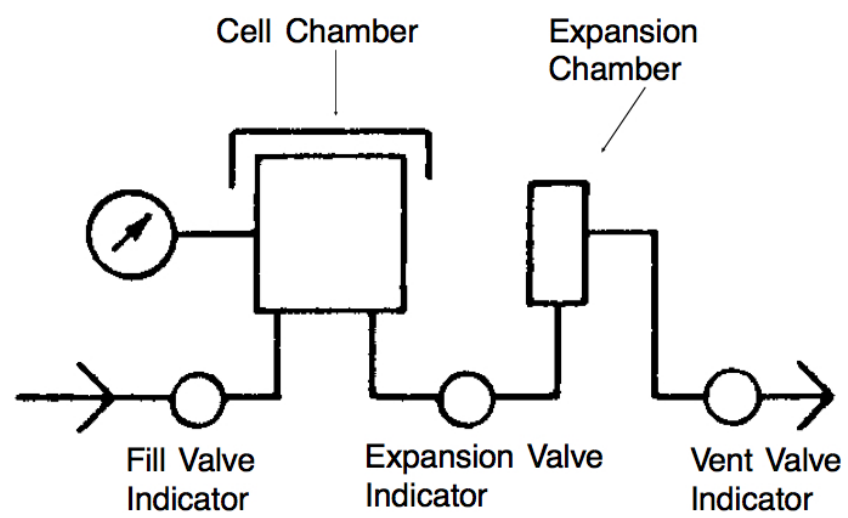

Figure 1.10: Schematic diagram of a gas displacement pycnometer. Reproduced from Micromeritics Instrument Corporation. ${ }^{79}$ 


\subsection{Small Scale Powder Disperser}

The Small Scale Powder Disperser (SSPD) from TSI Inc., Figure 1.11 effectively deagglomerates most dry particles in the diameter range from 0.5 to $50 \mu \mathrm{m}$. Powder dispersers are important for introducing powder into particle sizing systems, calibrating measurement instruments, and inhalation toxicology studies.

A small amount of sample is loaded within a ring on the turntable and the end of the stainless steel capillary of the venturi aspirator is positioned just above the turntable and sample. A suction transmitted through the capillary tube lifts particles from the turntable surface and small quantities of sample are dispersed. The air velocity in the venturi throat greatly exceeds that in the capillary tube causing shear forces where the two flows meet and the deagglomeration of the powder. The powder then enters an expansion cone, from which it exhausts from the unit. 


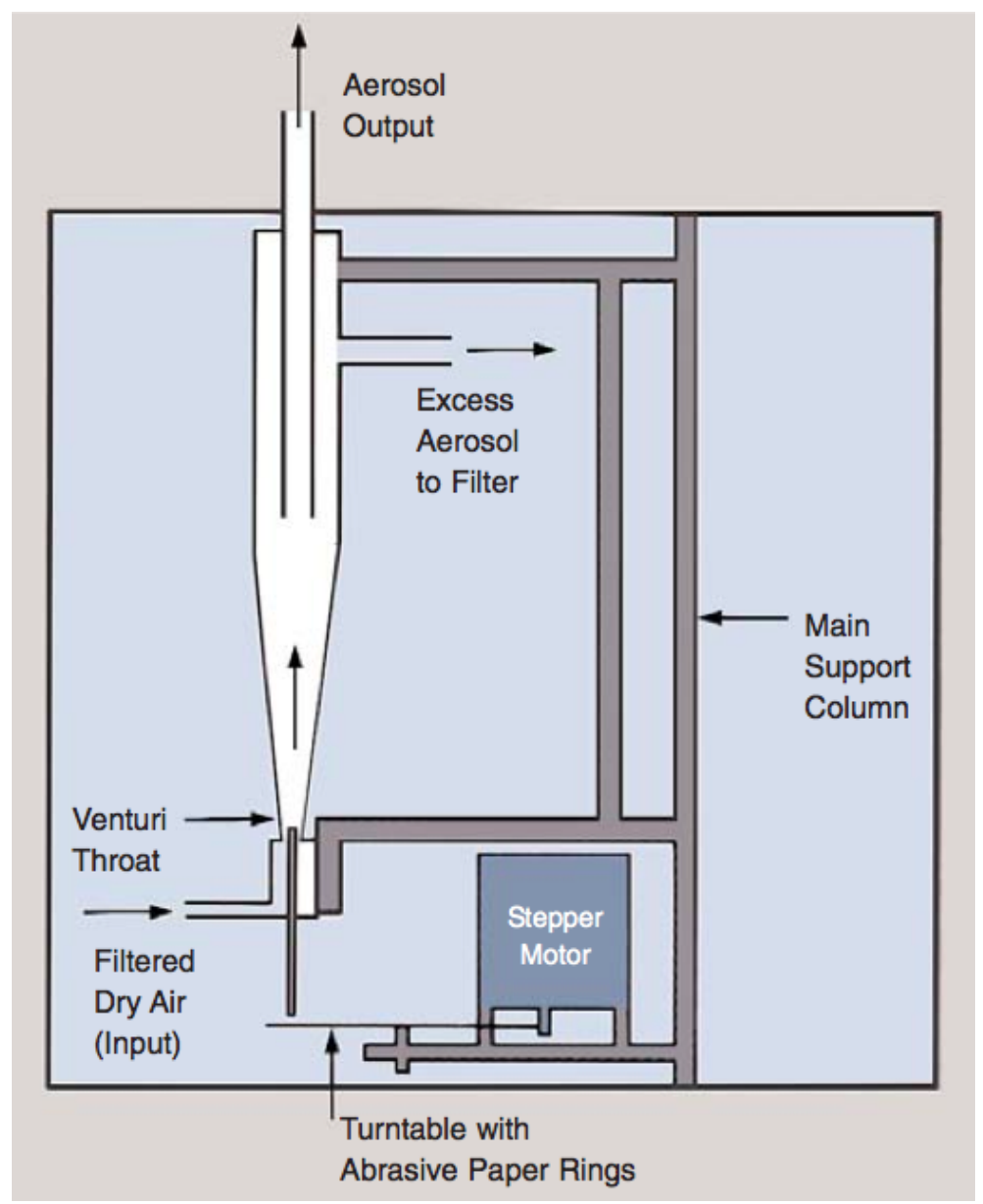

Figure 1.11: Schematic diagram of a small scale powder disperser. Reproduced from TSI Incorporated. 80 


\subsection{Aerodynamic Particle Sizer}

The only way of determining a particle's true aerodynamic behavior, whether a particle will penetrate a filter or be deposited in a lung, is to measure its aerodynamic diameter.

The Aerodynamic Particle Sizer (APS) Model 3320 from TSI Inc. measures the aerodynamic diameter of particles by measuring their time-of-flight between two laser beams. A particle entering the APS is accelerated through a nozzle with a small orifice. The particle then crosses the path of two laser beams, thus scattering light which is detected by a photodetector and converted into an electrical pulse, Figure 1.12, The time-of-flight between the two crests of these electrical pulses is measured and the particle's aerodynamic diameter is determined. Particles having a smaller aerodynamic diameter will accelerate rapidly and have shorter times-offlight. Conversely, particles having a larger aerodynamic diameter will accelerate slowly due to their increased inertia and have longer times-of-flight. Particles are resolved into various size groups, called bins and their corresponding concentrations are tabulated. 


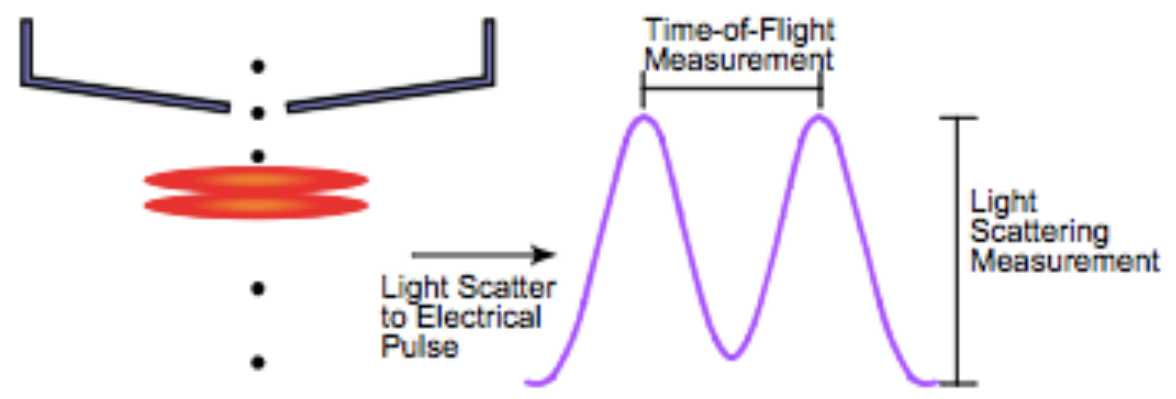

Figure 1.12: Schematic diagram of an aerodynamic particle sizer. Reproduced from TSI Incorporated. 81

\subsection{Micro-Orifice Uniform Deposit Impactor}

The Micro-Orifice Uniform Deposit Impactor (MOUDI) is a precision cascade impactor for collecting size-fractionated aerosol particle samples with applications in environmental monitoring, industrial hygiene studies, biological contamination, diesel and gasoline emission monitoring, and pharmaceutical inhalation research. MOUDI cascade impactors are characterized by their low inter-stages losses, low pressure drop, sharp cut efficiency curves, and stable predictable cut points.

The MOUDI is designed such that as the aerosol stream passes through each stage, particles having sufficient inertia will impact upon that particular stage collection plate, whilst smaller particles with insufficient inertia will follow the stream lines and pass through a nozzle to the next impaction stage. The stages are assembled in a stack in order of decreasing particle size (where the nozzles are smaller, the air velocity through the nozzles is higher and finer particles are collected) until the smallest particles are collected at an after-filter. The particle size distribution is determined by weighing the particles captured on each stage. If desired, the captured particles can also be used for size-fractionated chemical analysis.

The MOUDI used in this dissertation is comprised of ten stages (eleven including the inlet) with cut sizes at 18, 10, 5.6, 3.2, 1.8, 1.0, 0.56, 0.32, 0.18, 0.10 and 0.056 microns plus an after-filter. The nozzle and impaction plates rotate at $1 \mathrm{rpm}$ relative to each other, to spread out the particle deposit uniformly on each substrate and increasing the deposited particle mass without the risk of overloading. A vacuum pump generates the desired flow rate $(30 \mathrm{~L} / \mathrm{min})$ within the unit. 


\section{MOUDI ${ }^{\mathrm{TM}}$ Typical Stage}

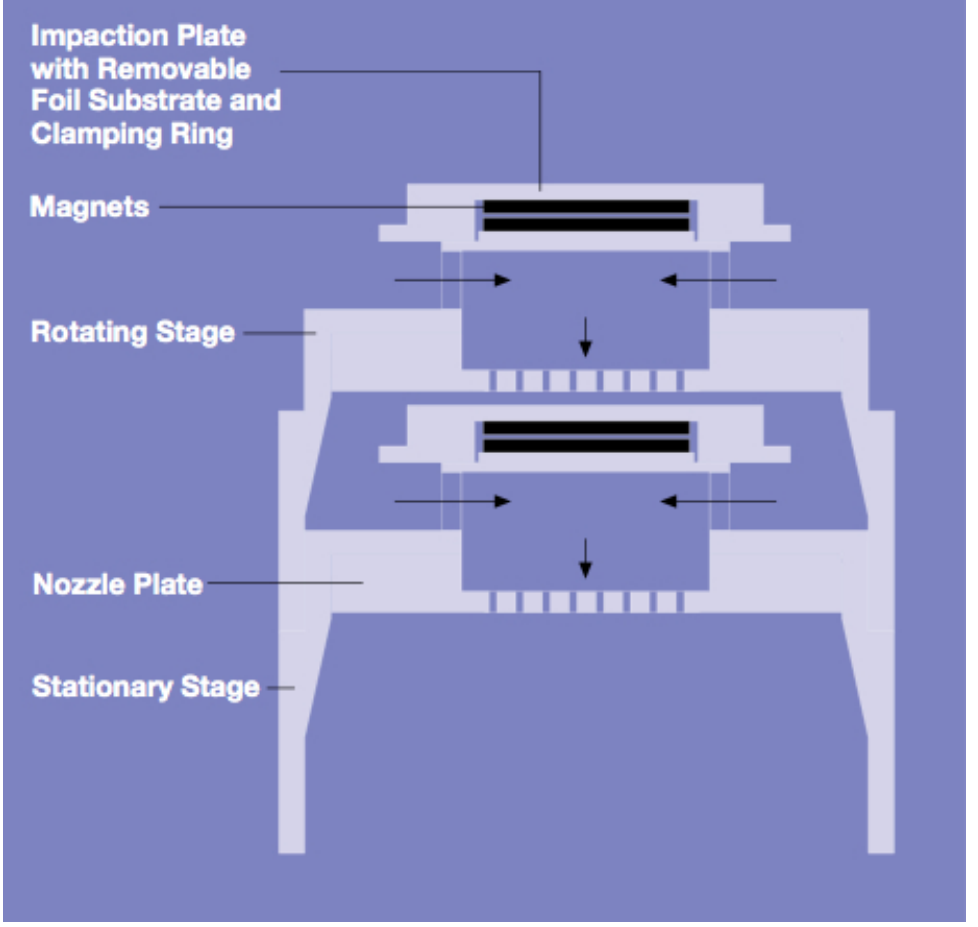

Figure 1.13: Schematic diagram of a typical MOUDI stage showing its relation to stage above and stage below. Reproduced from MSP Corporation. ${ }^{82}$ 


\subsection{Scanning Electron Microscope}

Like optical microscopes, Scanning Electron Microscopes (SEM) are used to study the surfaces of solid objects. A SEM is a different kind of microscope in that it uses electrons rather than light to form an image. Any item smaller than half the wavelength of light is too small to be seen with an optical microscope due to the diffraction limit of light. Electrons have a much shorter wavelength. Its advantages over an optical microscope include its high magnification and its large depth of field, which allows a large amount of the sample to be in focus at one time. For this reason, SEM produces images that are good representations of the sample's threedimensional shape.

A SEM uses a highly focused beam of electrons to form a magnified image of objects placed inside it. Where an optical microscope uses lenses to bend the light waves, in a SEM, electromagnets are used to bend the electron beam producing a beam of electrons that is focused to a tiny spot on the object that we wish to image. The SEM scans the focused electron beam across the surface of the sample in a rectangular pattern composed of a stacked series of linear scans (raster). At each point, the SEM records the brightness of the response signal. Changes in the composition (chemistry) and/or surface structure (morphology) of the sample will induce a varying amount of signal seen by the detector. In this way, a black and white SEM image of the sample is created.

The amount of magnification of the image is controlled by adjusting the size of the raster (i.e. the region over which it is scanned). Although magnifications of $10,000 \mathrm{x}$ are sufficient for most applications, a typical SEM can easily produce images with magnifications of 25,000x or higher. By comparison, a light microscope becomes increasingly difficult to use above $100 \mathrm{x}$ and is limited to an upper magnification of about 1000x.

All SEMs share the same essential components: the electron column, which houses the electron gun and electron lenses, the sample chamber, and the detector(s), as seen in Figure 1.14. Operation of a SEM requires a vacuum system, cooling system, vibration-free floor, and a room free of ambient magnetic and electric fields. At the top of the column is an electron gun, essentially a sharp tungsten filament. A strong electric field draws current off of that filament creating an electron beam. Below the electron gun is an anode that accelerates the electrons down the column, giving the incident electrons enough energy to eject electrons from the sample. Below the anode are condenser lenses to focus the electrons to a very fine spot, scanning coils for moving the beam back and forth across a sample, stigmators for adjusting the 
roundness of the beam, and an objective lens. The electron beam exits the probe lens and enters the sample chamber where it impacts the sample. The sample absorbs the energy of the electrons and re-emits Auger electrons, X-rays (that are used to show spatial variations in elemental composition), primary backscattered electrons (that are used to determine crystalline structure), and secondary electrons (that produce SEM images). Every SEM is equipped with a detector that counts these secondary electrons and sends the signal to an amplifier. The final image is built from the number of electrons emitted from each spot on the sample.

SEMs can be equipped to detect (i) secondary electrons, (ii) backscattered electrons, and (iii) X-rays. Every SEM is equipped to detect secondary electrons which provide variations in the morphology and surface topography. Backscattered electrons and X-rays are common options for detectors on a SEM. Backscattered electrons provide the average (atomic number) chemical makeup of the sample, whereas $\mathrm{X}$-rays measure the exact elements present and their relative concentrations.

FEI's Inspect Scanning Electron Microscope, used in this dissertation to image BG spores, is a high current Field Emission Gun (FEG) SEM equipped with a Schottky Field Emission source. 


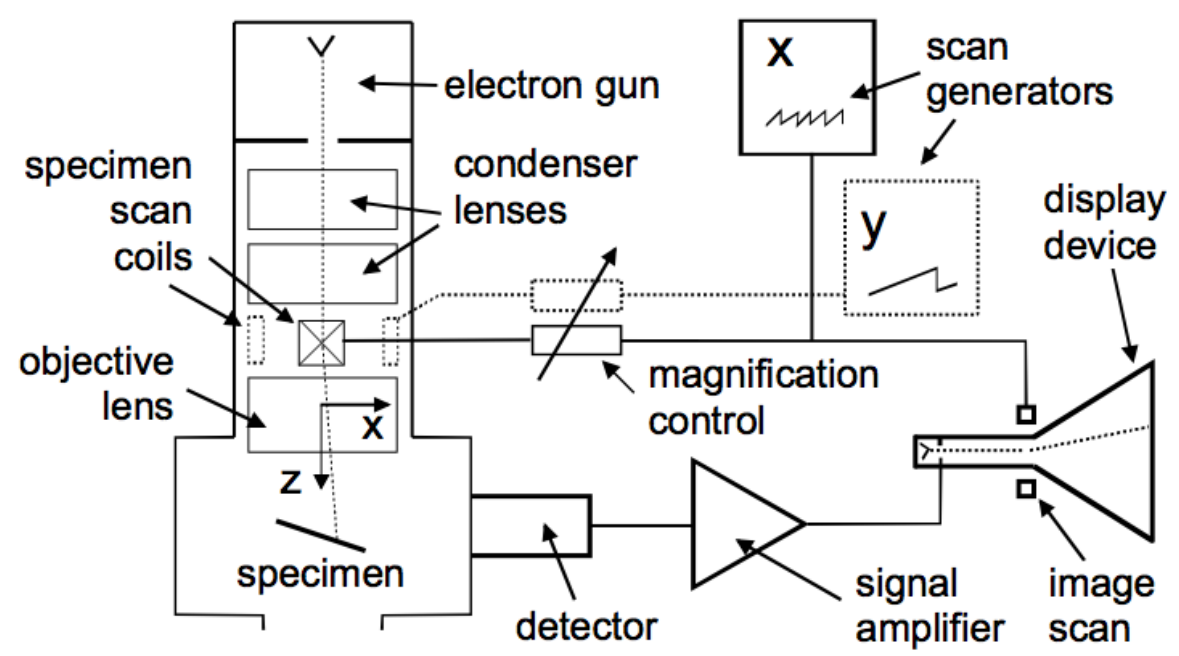

Figure 1.14: Schematic diagram of a scanning electron microscope. Reproduced from reference. .83

\subsection{Raman Spectroscopy}

Spectroscopy is the use of electromagnetic radiation to probe matter and interpret the results in order to characterize chemical structure. Within this category lies Raman spectroscopy - a inelastic light scattering technique in which a single wavelength of laser light interacts with the atoms and molecules that make up the sample to produce scattered radiation of different wavelengths. The change in wavelength between the incoming photon of light and the scattered radiation is extremely information rich, useful for the chemical identification and characterization of molecular structures. Raman spectroscopy allows for the fast, non-invasive, and non-destructive chemical analysis of solids, powders, and liquids within a wide range of applications, including pharmaceuticals, forensic science, polymers, and thin films.

In this technique, a sample is illuminated/irradiated with intense, monochromatic laser light in the visible or near-IR region. The light can be transmitted, reflected, absorbed, or scattered by species present in the sample. Scattering is a two-photon process in which an absorbed photon raises the molecule to a virtual excited state (lasting on the order of $10^{-14}$ seconds) and then re-emits a photon. The overwhelming majority of emitted photons do not gain or lose energy during scattering and thus have the same frequency as the incident radiation frequency, $v_{0}$. This scattering 
process is known as Rayleigh scattering. But a small amount of photons (approximately 1 in $10^{7}$ ) are emitted at a different frequency. A change in frequency of the light is called Raman scattering and can be of higher or lower energy than the incident light depending upon the vibrational state of the molecule. Exciting some vibrational mode of the molecule to give a lower scattered photon energy, $v_{0}-v_{v i b}$, is called Stokes Raman scattering. Whereas, scattering off an excited vibrational state of a molecule, which adds its vibrational energy to the incident photon, such that the scattered photon gains energy from the molecule, $v_{0}+v_{v i b}$, is known as Anti-Stokes Raman scattering.

Figure 1.15 shows an energy state diagram for Stokes, Rayleigh, and Anti-Stokes Raman scattering and the resulting Raman spectrum. The pattern of energy shifts (excitation wavelength plus or minus the vibrational mode of the bond) is the same for Stokes and Anti-Stokes. The Stokes signal is the stronger of the two, but it is still several orders of magnitude weaker than the Rayleigh line. Normally in Raman spectroscopy only the Stokes half of the spectrum is used, due to its greater intensity.

A spectrometer divides the scattered light into a spectrum of its frequency components, the peaks of which correspond to particular energy transitions (i.e. structural details). The energy shifts, typically in the range of $200-4000 \mathrm{~cm}^{-1}$, are expressed within a Raman spectrum as reciprocal centimeters $\left(\mathrm{cm}^{-1}\right)$ also called wavenumbers or Raman shifts. The result is a Raman spectrum - a graph of how much the molecules scatter the original light (i.e. intensity) versus wavenumber. The pattern of scattered photons is characteristic of the vibrational energy transitions of the particular molecular species involved. And because chemicals have unique chemical structures, they also have unique Raman spectra - in essence a molecular "fingerprint." And just like criminals are identified by searching human fingerprints in databases, the contents of a sample of unknown composition can be identified by matching Raman spectra from a library.

Although there can be striking similarities between IR spectra and Raman spectra, enough differences remain between the kinds of groups that are IR-active and Raman-active to make the techniques complementary rather than competitive. Infrared and Raman spectra are both due to molecular vibrations; within a spectrum, the peaks correspond to the energies of vibrating (stretching and bending) chemical bonds. However, each technique arrives at those vibrations via different mechanisms (absorption versus scattering), making each technology suited to different types of bonds. For a compound to be IR-active the molecule must undergo a change in dipole moment during vibration (water, alcohols, ketones, etc.), whereas for a compound to be Raman-active there must be a change in the polarizability of the molecule during 


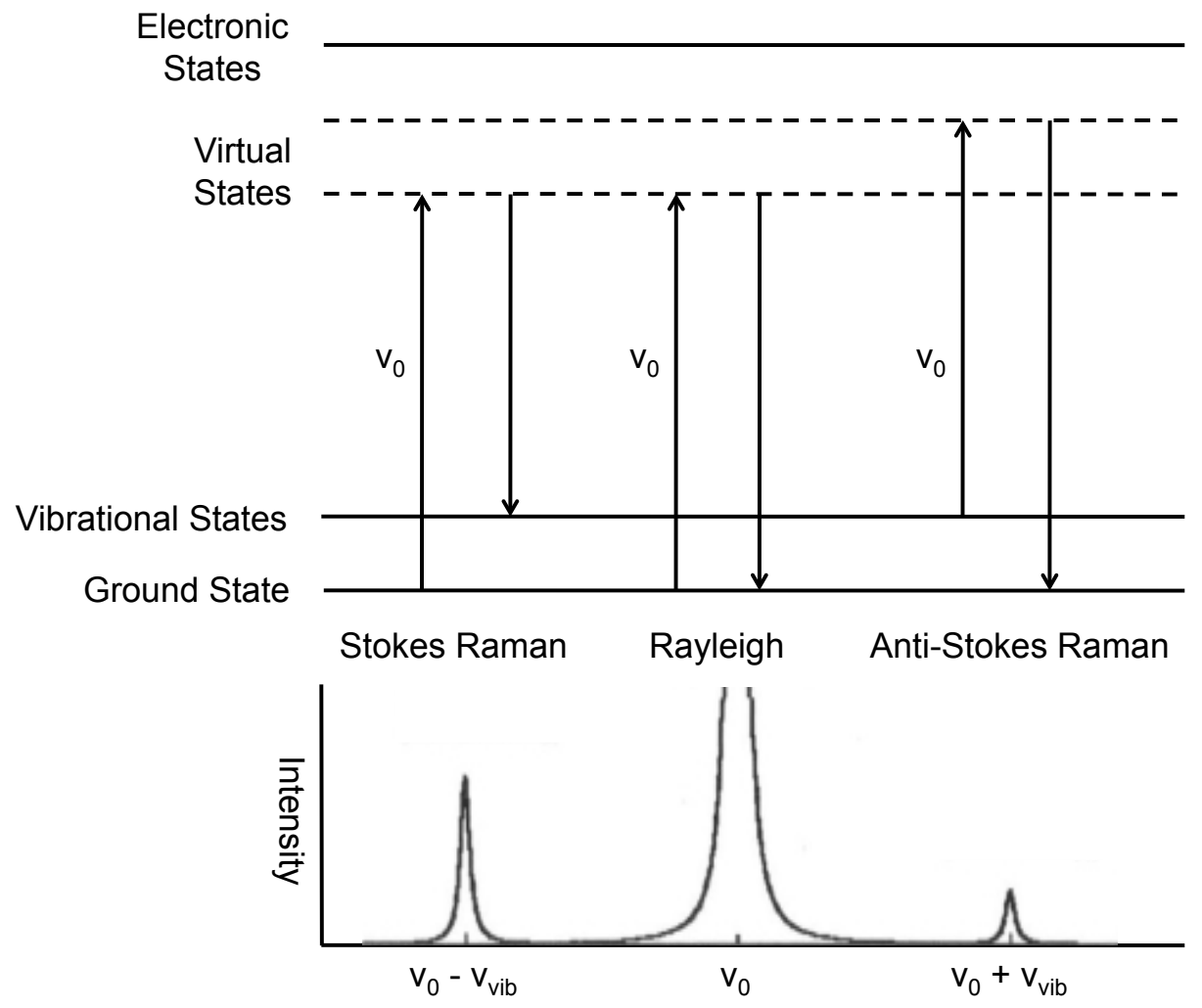

Figure 1.15: Energy state diagram showing the states involved in three different forms of scattering and their resulting spectrum, where $v_{0}$ is the initial frequency. 
the vibration (hydrocarbons, aromatics, etc.).

The Raman Systems R-3000 used in this dissertation is equipped with two wavelengths for use, 532 and $785 \mathrm{~nm}$. Shorter wavelengths generate Raman scattering more efficiently (the Raman scattering cross section depends on $\frac{1}{\text { wavelength }^{4}}$ ), thus producing a more intense signal. However, problems can arise when shorter wavelengths, such as in the visible region, are used. These higher energy sources can cause sample fluorescence, producing a signal that will swamp the weak inelastic scattering signal. Whereas, use of longer excitation wavelengths reduces the probability of interference from fluorescence in most samples. To compensate for the reduced scattering efficiency, longer integration times or higher power lasers can be used at these longer wavelengths. However, too much laser power can result in sample heating and degradation, resulting in poor spectra. Thus, having a number of laser wavelengths available for use may prove beneficial. 


\section{Bibliography}

[1] E. de Hoffmann and V. Stroobant, Mass Spectrometry: Principles and Applications. John Wiley \& Sons, Ltd., second ed., 2002.

[2] J. J. Thomson, Rays of Positive Electricity and Their Appplication to Chemical Analysis. Longmans Green and Co, 1913.

[3] F. W. Aston, "A positive-ray spectrograph," Philosophical Magazine, vol. 38, pp. 707-714, 1919.

[4] J. W. Coburn and E. Kay, "A new technique for the elemental analysis of thin surface layers of solids," Applied Physics Letters, vol. 19, no. 9, pp. 350-352, 1971.

[5] J. W. Coburn, E. Taglauer, and E. Kay, "Glow-discharge mass spectrometrytechnique for determining elemental composition profiles in solids," Journal of Applied Physics, vol. 45, no. 4, pp. 1779-1786, 1974.

[6] D. C. Duckworth, C. M. Barshick, and D. H. Smith, "Analysis of soils by glow discharge mass spectrometry," Journal of Analytical Atomic Spectrometry, vol. 8, pp. 875-879, 1993.

[7] M. Betti, G. Rasmussen, T. Hiernaut, and L. Koch, "Adaptation of a glow discharge mass spectrometer in a glove-box for the analysis of nuclear materials," Journal of Analytical Atomic Spectrometry, vol. 9, pp. 385-391, 1994.

[8] C. M. Barshick, D. H. Smith, J. H. Hackney, B. A. Cole, and J. W. Wade, "Glow discharge mass spectrometric analysis of trace metals in petroleum," Analytical Chemistry, vol. 66, pp. 730-734, 1994.

[9] L. Li, C. M. Barshick, J. T. Millay, A. V. Welty, and F. L. King, "Determination of bromine in flame-retardant plastics using pulsed glow discharge mass spectrometry," Analytical Chemistry, vol. 75, no. 16, pp. 3953-3961, 2003.

[10] T. E. Gibeau and R. K. Marcus, "Glow discharge ionization source for liquid chromatography/particle beam mass spectrometry," Analytical Chemistry, vol. 72, no. 16, pp. 3833-3840, 2000.

[11] W. C. Davis, J. L. Venzie, B. Willis, R. L. Coffee Jr., D. P. Arya, and R. K. Marcus, "Particle beam glow discharge mass spectrometry: spectral characteristics of nucleobases," Rapid Communications in Mass Spectrometry, vol. 17, pp. 1749-1758, 2003. 
[12] J. C. Derrick Quarles, S. Niemann, and R. K. Marcus, "Conversion of a commercial gas chromatography-mass spectrometer to a liquid chromatography-particle beam/glow discharge mass spectrometer," Journal of Analytical Atomic Spectrometry, vol. 25, pp. 1780-1786, 2010.

[13] C. M. Barshick, S. A. Barshick, M. L. Mohill, P. F. Britt, and D. H. Smith, "Elemental and organometallic analyses of soil using glow discharge mass spectrometry and gas chromatography/mass spectrometry," Rapid Communications in Mass Spectrometry, vol. 10, no. 3, pp. 341-346, 1996.

[14] J. John P. Guzowski and G. M. Hieftje, "Gas sampling glow discharge: a versatile ionization source for gas chromatography time-of-flight mass spectrometry," Analytical Chemistry, vol. 72, no. 16, pp. 3812-3820, 2000.

[15] C. L. Lewis, M. A. Moser, D. E. Dale Jr., W. Hang, C. Hassell, F. L. King, and V. Majidi, "Time-gated pulsed glow discharge: real-time chemical speciation at the elemental, structural, and molecular level for gas chromatography time-offlight mass spectrometry," Analytical Chemistry, vol. 75, no. 9, pp. 1983-1996, 2003.

[16] K. Newman and R. S. Mason, "Gas chromatography combined with fast flow glow discharge mass spectrometry (gc-ffgd-ms)," Journal of Analytical Atomic Spectrometry, vol. 19, pp. 1134-1140, 2004.

[17] B. Chapman, Glow Discharge Processes: Sputtering and Plasma Etching. New York: Wiley, 1980.

[18] R. K. Marcus, ed., Glow Discharge Spectroscopies. Plenum Press, 1993.

[19] W. W. Harrison, C. Yang, and E. Oxley, Glow Discharge Plasmas in Analytical Spectroscopy. John Wiley \& Sons, Ltd., 2003.

[20] W. W. Harrison, K. R. Hess, R. K. Marcus, and F. L. King, "Glow discharge mass spectrometry," Analytical Chemistry, vol. 58, no. 2, pp. 341A-356A, 1986.

[21] W. W. Harrison, C. M. Barshick, J. A. Klingler, P. H. Ratliff, and Y. Mei, "Glow discharge techniques in analytical chemistry," Analytical Chemistry, vol. 62, no. 18, pp. 943A-949A, 1990.

[22] A. Bogaerts and R. Gijbels, "Fundamental aspects and applications of glow discharge spectrometric techniques," Spectrochimica Acta Part B, vol. 53, pp. 142, 1998. 
[23] M. R. Winchester and R. Payling, "Radio-frequency glow discharge spectrometry: A critical review," Spectrochimica Acta Part B, vol. 59, pp. 607-666, 2004.

[24] V. Hoffmann, M. Kasik, P. K. Robinson, and C. Venzago, "Glow discharge mass spectrometry," Analytical and Bioanalytical Chemistry, vol. 381, pp. 173-188, 2005.

[25] N. Jakubowski, R. Dorka, E. Steers, and A. Tempez, "Trends in glow discharge spectroscopy," Journal of Analytical Atomic Spectrometry, vol. 22, pp. 722-735, 2007.

[26] R. Pereiro, A. Sola-Vazquez, L. Lobo, J. Pisonero, N. Bordel, J. M. Costa, and A. Sanz-Medel, "Present and future of glow discharge - time of flight mass spectrometry in analytical chemistry," Spectrochimica Acta Part B, vol. 66, pp. 399412, 2011.

[27] D. A. Swift, "The electrical discharge," Contemporary Physics, vol. 22, no. 1, pp. 37-60, 1981.

[28] F. M. Penning, Electrical Discharges in Gases. New York: Wiley, 1957.

[29] A. M. Howatson, An introduction to gas discharges. Pergamon Press (Oxford, New York), 1976.

[30] A. Bogaerts, M. van Straaten, and R. Gijbels, "Monte carlo simulation of an analytical glow discharge: motion of electrons, ions and fast neutrals in the cathode dark space," Spectrochimica Acta, vol. 50B, no. 2, pp. 179-196, 1995.

[31] Y. P. Raizer, Gas Discharge Physics. Springer, 1997.

[32] R. A. Meyers, ed., Encyclopedia of Analytical Chemistry. John Wiley \& Sons, Ltd., 2009.

[33] F. L. King and W. W. Harrison, "Glow discharge mass spectrometry: an introduction to the technique and its utility," Mass Spectrometry Reviews, vol. 9, pp. 285-317, 1990.

[34] M. Ohring, The Materials Science of Thin Films. Academic Press, Inc., 1992.

[35] D. Fang and R. K. Marcus, "Parametric evaluation of sputtering in a planar, diode glow discharge. i. sputtering of oxygen-free hard copper (OFHC)," Spectrochimica Acta, Part B: Atomic Spectroscopy, vol. 43B, no. 12, pp. 1451-1460, 1988. 
[36] H. Helm, K. Stephan, and T. D. Mark, "Electron-impact ionization of $\mathrm{Ar}^{2}$, ArKr, $\mathrm{Kr}_{2}$, KrXe, and $\mathrm{Xe}_{2}$," Physical Review A, vol. 19, no. 6, pp. 2154-2160, 1979.

[37] I. Abril, "Modeling of glow discharge sputtering systems," Computer Physics Communications, vol. 51, pp. 413-422, 1988.

[38] J. A. Klingler, P. J. Savickas, and W. W. Harrison, "The pulsed glow discharge as an elemental ion source," Journal of the American Society for Mass Spectrometry, vol. 1, no. 2, pp. 138-143, 1990.

[39] F. L. King and C. Pan, "Temporal signal profiles of analytical species in modulated glow discharge plasmas," Analytical Chemistry, vol. 65, no. 6, pp. 735-739, 1993.

[40] W. Hang, C. Baker, B. W. Smith, J. D. Winefordner, and W. W. Harrison, "Microsecond-pulsed glow discharge time-of-flight mass spectrometry: analytial advantages," Journal of Analytical Atomic Spectrometry, vol. 12, no. 2, pp. 143149, 1997.

[41] V. Majidi, M. A. Moser, C. L. Lewis, W. Hang, and F. L. King, "Explicit chemical speciation by microsecond pulsed glow discharge time-of-flight mass spectrometry: concurrent acquisition of structural, molecular and elemental information," Journal of Analytical Atomic Spectrometry, vol. 15, no. 1, pp. 19-25, 2000.

[42] F. L. King, "Direct determination of trace elements in graphite matrices using modulated glow discharge atomic absorption spectrometry," Applied Spectroscopy, vol. 47, no. 3, pp. 300-304, 1993.

[43] W. Hang, W. O. Walden, and W. W. Harrison, "Microsecond pulsed glow discharge as an analytical spectroscopic source," Analytical Chemistry, vol. 68, no. 7, pp. 1148-1152, 1996.

[44] D. Serxner, R. L. Smith, and K. R. Hess, "Investigations of a metastable dependence on the ionization of sputtered species in neon glow discharges," Applied Spectroscopy, vol. 45, no. 10, pp. 1656-1664, 1991.

[45] E. B. M. Steers and R. J. Fielding, "Charge-transfer excitation processes in the Grimm lamp," Journal of Analytical Atomic Spectrometry, vol. 2, pp. 239-244, 1987. 
[46] C. L. Lewis, G. P. Jackson, S. K. Doorn, V. Majidi, and F. L. King, "Spectral, spatial and temporal characterization of a millisecond pulsed glow discharge: copper analyte emission and ionization," Spectrochimica Acta Part B, vol. 56, pp. 487-501, 2001.

[47] M. van Straaten, A. Bogaerts, and R. Gijbels, "Experimental determination of the energy distribution of ions bombarding the cathode surface in a glow discharge," Spectrochimica Acta, vol. 50B, no. 4-7, pp. 583-605, 1995.

[48] E. E. Muschlitz Jr., "Metastable atoms and molecules," Science, vol. 159, pp. 599-604, 1968.

[49] K. R. Hess and W. W. Harrison, "The role of metastable atoms in glow discharge ionization processes," Analytical Chemistry, vol. 60, pp. 691-696, 1988.

[50] R. L. Smith, D. Serxner, and K. R. Hess, "Assessment of the relative role of penning ionization in low-pressure glow discharges," Analytical Chemistry, vol. 61, no. 10, pp. 1103-1108, 1989.

[51] W. Hang and W. W. Harrison, "Diffusion, ionization, and sampling processes in the glow discharge source for mass spectrometry," Analytical Chemistry, vol. 69, no. 24, pp. 4957-4963, 1997.

[52] C. Pan and F. L. King, "Ion formation processes in the afterpeak time regime of pulsed glow discharge plasmas," Am Soc Mass Spectrom, vol. 4, pp. 727-732, 1993.

[53] C. L. Lewis, E. S. Oxley, C. K. Pan, R. E. Steiner, and F. L. King, "Determination of ${ }^{40} \mathrm{Ca}^{+}$in the presence of ${ }^{40} \mathrm{Ar}^{+}$: An illustration of the utility of time-gated detection in pulsed glow discharge mass spectrometry," Analytical Chemistry, vol. 71, pp. 230-234, 1999.

[54] R. E. Steiner, C. L. Lewis, and F. L. King, "Time-of-flight mass spectrometry with a pulsed glow discharge ionization source," Analytical Chemistry, vol. 69, no. 9, pp. 1715-1721, 1997.

[55] R. J. Cotter, Time-of-Flight Mass Spectrometry: Instrumentation and Applications in Biological Research. Washington, DC: American Chemical Society, 1997. 
[56] W. C. Wiley and I. H. McLaren, "Time-of-flight mass spectrometer with improved resolution," Review of Scientific Instruments, vol. 26, no. 12, pp. 1150$1157,1955$.

[57] R. J. Cotter, "Time-of-flight mass spectrometry: An increasing role in the life sciences," Biomedical and Environmental Mass Spectrometry, vol. 18, pp. 513$532,1989$.

[58] G. J. O'Halloran, L. W. Walker, R. A. Fluegge, J. F. Belts, and W. L. Everett, "Determination of chemical species prevalent in a plasma jet," technical report, Bendix Corporation Research Laboratories, Southfield Michigan. Air Force Materials Laboratories, Research and Technology Division, Air Force Systems Command, United States Air Force, 1964.

[59] M. Guilhaus, D. Selby, and V. Mlynski, "Orthogonal acceleration time-of-flight mass spectrometry," Mass Spectrometry Reviews, vol. 19, pp. 65-107, 2000.

[60] J. H. J. Dawson and M. Guilhaus, "Orthogonal-acceleration time-of-flight mass spectrometer," Rapid Communications in Mass Spectrometry, vol. 3, pp. 155$159,1989$.

[61] F. Rouessac and A. Rouessac, Chemical Analysis: Modern Instrumentation Methods and Techniques. West Sussex, England: John Wiley \& Sons, Ltd., 2000.

[62] P. B. O'Connor and F. Hillenkamp, MALDI MS: A Practical Guide to Instrumentation, Methods and Applications, ch. 2: MALDI Mass Spectrometry Instrumentation. Wiley-VCH, 2007.

[63] A. J. P. Martin and R. L. M. Synge, "A new form of chromatogram employing two liquid phases," Biochemical Journal, vol. 35, pp. 1358-1368, 1941.

[64] A. T. James and A. J. P. Martin, "Gas-liquid partition chromatography: the separation and micro-estimation of volatile fatty acids from formic acid to dodecanoic acid," Biochemical Journal, vol. 50, pp. 679-690, 1952.

[65] K. D. Bartle and P. Myers, "History of gas chromatography," Trends in Analytical Chemistry, vol. 21, 2002.

[66] M. J. E. Golay, "Gas chromatography," in Lansing Symposium, 1957.

[67] M. J. E. Golay, "Gas chromatography," in Amsterdam Symposium, 1958. 
[68] L. S. Ettre, "Evolution of capillary columns for gas chromatography," $L C G C$ : Milestones in Chromatography, vol. 19, no. 1, pp. 48-59, 2001.

[69] R. D. Dandeneau and E. H. Zerenner, "An investigation of glasses for capillary chromatography," Journal of Separation Science, vol. 2, no. 6, pp. 351-356, 1979 .

[70] J. Pawliszyn, Solid Phase Microextraction: Theory and Practice. Wiley, 1997.

[71] H. Prosen and L. Zupancic-Kralj, "Solid-phase microextraction," TrAC Trends in Analytical Chemistry, vol. 18, no. 4, pp. 272-282, 1999.

[72] J. Pawliszyn, "Theory of solid-phase microextraction," Journal of Chromatographic Science, vol. 38, pp. 270-278, 2000.

[73] J. V. Hinshaw, "Solid-phase microextraction," LCGC: GC Connections, pp. 2$5,2003$.

[74] G. Vas and K. Vekey, "Solid-phase microextraction: a powerful sample preparationg tool prior to mass spectrometric analysis," Journal of Mass Spectrometry, vol. 39, pp. 233-254, 2004.

[75] J. Pawliszyn, ed., Handbook of Solid Phase Microextraction. Elsevier Inc., 2012.

[76] C. L. Arthur and J. Pawliszyn, "Solid phase microextraction with thermal desorption using fused silica optical fibers," Analytical Chemistry, vol. 62, no. 19, pp. 2145-2148, 1990.

[77] D. Louch, S. Motlagh, and J. Pawliszyn, "Dynamics of organic compound extraction from water using liquid-coated fused silica fibers," Analytical Chemistry, vol. 64, no. 10, pp. 1187-1199, 1992.

[78] <http://www.sigmaaldrich.com/content/dam/sigmaaldrich/docs/Supelco/Brochure/1/supelco-7-spme.pdf $>$ accessed 13 July 2013.

[79] Micromeritics Instrument Corporation, One Micromeritics Drive, Norcross, GA

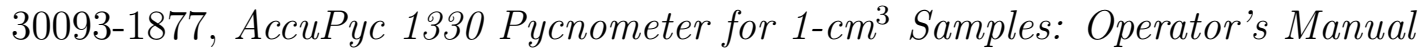
V3.03, September 2006.

[80] TSI Incorporated, 500 Cardigan Road, Shoreview, MN 55126, Model 3433 Small-Scale Powder Disperser: Instruction Manual, February 2003. 
[81] TSI Incorporated, 500 Cardigan Road, St. Paul, MN 55164, Model 3320 Aerodynamic Particle Sizer Spectrometer, July 2000.

[82] MSP Corporation, 5910 Rice Creek Parkway, Shoreview, MN 55126, MOUDIMicro-Orifice, Uniform-Deposit Impactor Models 100 and 110, 1999.

[83] R. F. Egerton, Physcial Principles of Electron Microscopy: An Introduction to TEM, SEM, and AEM. Springer Science, 2005. 


\section{Chapter 2}

\section{Ion Formation in Millisecond Pulsed Glow Discharge Plasmas*}

*J. Anal. At. Spectrom., 2011, 26, 2206-2215 - Reproduced by permission of The Royal Society of Chemistry

<http://pubs.rsc.org/en/content/articlepdf/2011/ja/c1ja10102a>

\subsection{Abstract}

Ion intensity profiles, for both discharge gas $\left({ }^{40} \mathrm{Ar}^{2+},{ }^{40} \mathrm{Ar}^{+},{ }^{40} \mathrm{Ar}_{2}^{+}\right)$and sputtered species $\left({ }^{63} \mathrm{Cu}^{+}\right)$, have been measured for a series of parameters including sampling distance, pulse power, discharge gas pressure, pulse width, and duty cycle in a millisecond pulsed direct current (dc) glow discharge plasma using time-gated detection with a time-of-flight (TOF) mass spectrometer. Throughout these experiments constant power was maintained for comparative profile measurements. Intensity profiles for both discharge gas and sputtered material were constructed using the intensity values from a compilation of over 100 mass spectra. Ion signals from analytically important (sputtered) species differed in their response to changes in sampling distance, discharge gas pressure, pulse width, and duty cycle than those of discharge gas species. These intensity profiles provide insight into the effects of discharge conditions on the time-dependent behavior of different ions in the plasma. In addition to using time-gated acquisition, it is possible to further influence ion formation within the plasma through the careful selection of these plasma parameters; in doing so, one can maximize sputtered ion signals while suppressing ion signals from discharge gas 
species. In this research, we conduct a comparative investigation of ion signal temporal profiles through the variation of discharge parameters to better refine the method and to gain a better understanding of the processes taking place in the discharge.

\subsection{Introduction}

Glow discharge (GD) ionization is a powerful analytical tool, well suited to the direct analysis and depth-profiling of conductive and non-conductive solid samples, which typically function as the cathode of the system. Glow discharges that are pulsed consist of an applied square wave power pulse, microseconds ( $\mu s$ ) to milliseconds (ms) in length, followed by a relatively longer period of power termination. With respect to steady-state glow discharges, pulsed glow discharges offer the advantages of increased atomization and ionization, arising from the application of high peak voltages and currents, and reduced plasma instability, arising from the alleviation of overheating of the sample cathode. The key analytical advantage gained from pulsing a glow discharge plasma is the formation of different time regimes, termed the 'prepeak', 'plateau', and 'afterpeak', in which either electron ionization or Penning ionization can be enhanced or suppressed. In addition to selecting a certain time during the plasma's pulse cycle to acquire data, conditions can be chosen to control the plasma chemistry and thus the ionization to get specific energy into a molecule and thus specific fragments. To derive these operating conditions of a pulsed glow discharge ionization source, it is necessary to know and understand the effects that various operating parameters have on ionization.

Studies of discharge parameters have been reported by previous investigators. 1 . 7 An earlier work using atomic absorption spectrometry and mass spectrometry was described by Loving and Harrison, ${ }^{8}$ wherein cathode to anode distance and discharge pressure were examined. This work was done at constant current. Klinger et. al. previously studied the effect of $25 \%, 50 \%$, and $75 \%$ duty cycles on the size of the afterpeak. ${ }^{1}$ In their study the duty cycle was decreased, but in order to maintain a constant current over the pulse cycle, the applied voltage was increased. A study of a pulsed, radio-frequency glow discharge by atomic absorption spectrophotometry by Parker and Marcus ${ }^{9}$ evaluated the roles of power, pressure, orifice diameter, plasma duty cycle, and GD pulse width in the production of gas-phase sample atoms while using constant discharge power. Small duty cycles showed an increase in the production of ground-state atoms. However, as the duty cycle was decreased, the same overall average power was maintained, resulting in higher instantaneous powers. This 
yielded higher afterpeak intensities most likely due to the increase in applied power, not as the result of a smaller duty cycle. More recently, Muniz et. al. have shown that there are even differences between different sputtered analytes as a function of discharge operating conditions. ${ }^{7}$ Most of the parametric pulsed glow discharge work to date has been done under constant discharge voltage, current, or average power (over the entire cycle). Whereas here, measurements were obtained using constant instantaneous discharge power. Furthermore, this research differs from these previous studies by examining doubly charged and dimer discharge gas ions in addition to the signal from the singly charged discharge gas ion and sputtered cathode ions.

For a parametric study of diverse experiments, maintaining constant voltage or constant current would not serve as well as maintaining constant power for comparative measurements ${ }^{10}$ of sputtered species. The intensity of the ion signal of sputtered species is normally proportional to the their sputtered atom density. ${ }^{8}$ In turn, sputtered atom populations are largely defined by the sputter energy and rate of atom bombardment. ${ }^{3}$ The applied voltage of the pulsed discharge determines the kinetic energy of the atoms and ions that impact the cathode surface. The higher the voltage, the greater the number of atoms released from the cathode surface as a result of the impact of a single primary ion, what is known as sputter yield; 11 whereas, the applied current is positively related to the rate at which the cathodic material is sputtered. It is the combination of these two, voltage and current, that produces the sputtered atom population. $\frac{14}{14}$ This fact becomes important with abnormal glow discharges, in which voltage and current increase proportionally. When maintaining a constant discharge current, the voltage will be affected by changes in distance from cathode to ion exit orifice and changes in pressure due to changes in the number of charge carriers between cathode and anode: the greater the distance or the higher

the pressure, the lower the voltages. ${ }^{8}$ Signal profiles observed in this research are thus a result of controlling the GD pulse power. Except where indicated, constant power was maintained for the following measurements, normalizing the population of neutral copper atoms generated during sputtering.

\subsection{Materials and methods}

\subsubsection{Glow discharge ion source}

The configuration of the de glow discharge ion source along with the sample introduction system and direct insertion probe used in this work is shown in Figure 2.1. The 
glow discharge chamber consists of a stainless steel six-way cross, fitted with conflat flanges. The copper cathode is mounted onto the dc direct insertion probe and introduced into the glow discharge chamber (which also served as the anode) through a ball valve fitted with a $1 / 2$ inch adapter that allows the probe to be inserted without breaking the vacuum. Sampling distances were taken to be the distance between the copper cathode and the ion exit orifice. In other words, the greater the distance, the further the cathode was positioned from the ion exit orifice.

The $5 \mathrm{~mm}$ diameter copper cathode is surrounded by a macor shield (ceramic insulator). This insulator has the effect of localizing the plasma onto the flat surface of the cathode. A square-wave dc pulse was applied to the cathode which was pulsed between ground and a high negative potential. A digital delay generator (DDG) (Model DG535, Stanford Research Systems, Inc.) was used to control the pulse width and frequency by gating an electronic pulser (Model PVX-4140, DEI) that converted dc power (Model PS350, Stanford Research Systems, Inc.) into a series of high voltage pulses. The glow discharge was operated in the constant power mode. The power was adjusted by controlling the voltage of the discharge pulse and monitoring the product of the applied voltage and instantaneous current. Pulse voltages were adjusted to $700-1700 \mathrm{~V}$ with pulse currents of 1-3 $\mathrm{mA}$ to yield pulse powers of 1.0-2.5 W. The voltage of the discharge was remotely controlled using LabVIEW (LabVIEW 7.1, National Instruments). The current indicated on a digital display of the power supply was the average current over the entire pulse cycle, therefore, the instantaneous current of the 'on' portion of the pulse cycle was calculated to account for the different duty cycles. The glow discharge chamber was filled with ultra high purity grade argon gas (Scott Specialty Gases) at operating pressures of 0.30, 0.60, and 0.90 Torr as monitored by a thermocouple gauge (Active gauge, Edwards).

\subsubsection{Time of flight mass analyzer}

The time-of-flight mass spectrometer used in this study was designed and constructed in house (Figure 2.1). Argon and sputtered species exit the glow discharge chamber through an ion exit orifice $(1 \mathrm{~mm}$ i.d.) and enter the skimmer cone $(1 \mathrm{~mm}$ i.d.) which selects a portion of the beam for transmission to the ion optics of the highvacuum segment of the mass spectrometer. Ions transmitted through the skimmer cone were focused without changing the ion energies into the repeller region of the mass analyzer by means of a three-tube Einzel lens assembly and ion slit $(2.5 \mathrm{~mm}$, fixed at ground potential). A second digital delay generator (Model 9650A, Signal Recovery), synchronized to the DDG of the pulsed glow discharge power supply, 


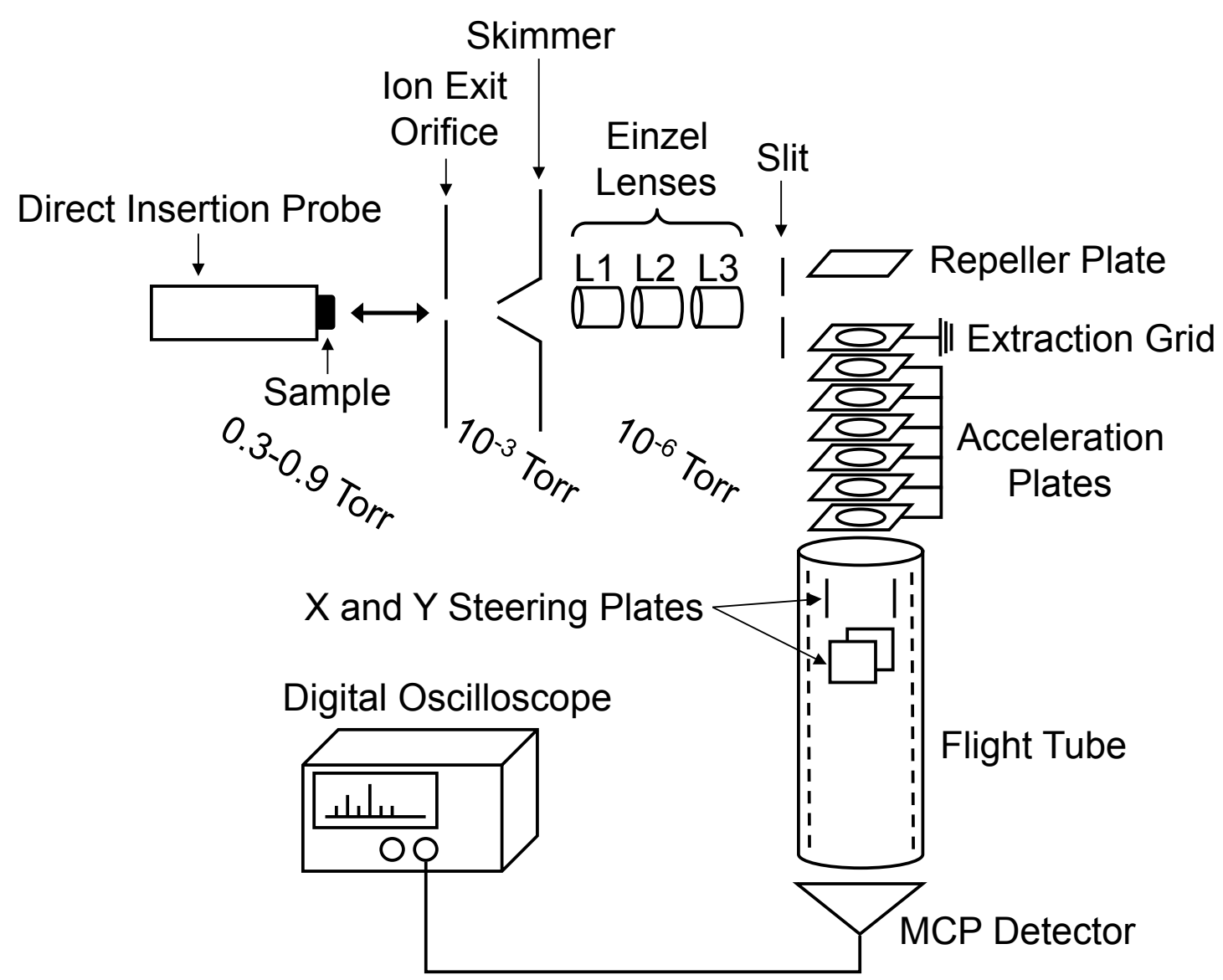

Figure 2.1: Diagram of glow discharge ion source with time-of-flight mass spectrometer. 
gated a second electronic pulser and dc power supply to provide the repeller pulse. The repeller plate was pulsed $2 \mu$ s per cycle with a positive potential to orthogonally inject a packet of ions into the flight tube. Adjustment of the gate delay position was accomplished using the repeller plate's DDG. By delaying the $2 \mu$ s repeller pulse with respect to the initiation of the $m s$ cathodic pulse, different portions of the GD pulse cycle could be sampled. The lens stack used for ion injection was constructed using a series of 2 inch square stainless steel plates each with a 1 inch diameter grid (Kimball Physics). General operating conditions for the GD-TOF mass spectrometer balancing ion transmission and resolution are listed in Table 2.1. The lens settings of the time-of-flight mass analyzer were optimized for each set of experiments.

Differential pumping of the instrument (provided by two turbomolecular pumps) yielded pressures of $0.30-0.90$ Torr in the glow discharge chamber, $10^{-3}$ Torr in the ion focusing region (first vacuum stage), and $10^{-6}$ Torr in the flight tube (second vacuum stage), enabling the sampling and subsequent analysis of the glow discharge plasma by time-of-flight mass spectrometry.

\subsubsection{Data collection}

Mass spectra were acquired using a digital oscilloscope (Waverunner LT372, LeCroy) connected to the micro-channel plate detector of the TOF. Each mass spectrum recorded was a result of 50 spectra averaged by the oscilloscope, each consisting of 10,000 data points. Figure 2.2 is a sample of four mass spectra taken at various times over the pulse cycle. They illustrate (a) a population inversion in which doubly charged argon is of higher intensity than singly charged argon, (b) the prepeak, where doubly charged argon decreases and singly charged argon becomes the dominant species, while our sputtered ion, $\mathrm{Cu}^{+}$, is still absent, (c) the plateau, where spectra are similar in appearance to that of a steady-state glow discharge, and (d) the afterpeak, where our sputtered ion, $\mathrm{Cu}^{+}$, experiences a surge in intensity.

The TOF repeller pulse was positioned at various times over the GD pulse cycle and mass spectra were collected at each position. During the plasma turn-on stage, spectra were collected from 0 to $0.050 \mathrm{~ms}$ in $0.002 \mathrm{~ms}$ increments and from 0.050 to $1.000 \mathrm{~ms}$ in $0.050 \mathrm{~ms}$ increments. During the plateau, spectra were collected from $1.000 \mathrm{~ms}$ to pulse termination in $0.100 \mathrm{~ms}$ increments for a $2.5 \mathrm{~ms}$ pulse, in 0.200 ms increments for a $5.0 \mathrm{~ms}$ pulse, and in $0.250 \mathrm{~ms}$ increments for a $7.5 \mathrm{~ms}$ pulse. From pulse termination to $0.200 \mathrm{~ms}$ later, mass spectra were collected in $0.010 \mathrm{~ms}$ increments, and from $0.200 \mathrm{~ms}$ to $2.500 \mathrm{~ms}$ following pulse termination in $0.050 \mathrm{~ms}$ increments using LabVIEW. A total of 126, 131, and 137 spectra were collected over 
Table 2.1: Pulsed GD-TOFMS Operating Conditions

\begin{tabular}{ll}
\hline Pulsed Glow Discharge & \\
\hline Cathode material & Copper \\
Pressure: discharge gas (Ar) & $0.30-0.60$ Torr \\
Pulse power & $1.0-2.0 \mathrm{~W}$ \\
Pulse width & $2.5-7.5 \mathrm{~ms}$ \\
Pulse duty cycle & $25-75 \%$ \\
Distance from cathode to ion exit orifice & $2-12 \mathrm{~mm}$ \\
\hline Time-of-Flight Mass Analyzer & \\
\hline 1st vacuum stage pressure & $10^{-3} \mathrm{Torr}$ \\
2nd vacuum stage pressure & $10^{-6} \mathrm{Torr}$ \\
Repeller pulse width & $2 \mu \mathrm{s}$ \\
Skimmer potential & $-500 \mathrm{~V}$ \\
Slit potential & ground \\
L1 potential & $-150 \mathrm{~V}$ \\
L2 potential & $-450 \mathrm{~V}$ \\
L3 potential & $-150 \mathrm{~V}$ \\
Repeller potential & $+200 \mathrm{~V}$ \\
Repeller bias potential & $-2 \mathrm{~V}$ \\
Extractor grid potential & ground \\
Accelerator / Flight tube potential & $-2000 \mathrm{~V}$ \\
X steering plate potential & $-2250 \mathrm{~V}$ \\
Y steering plate potential & $-2050 \mathrm{~V}$ \\
Micro-channel plate detector potential & $-1850 \mathrm{~V}$ \\
\hline
\end{tabular}




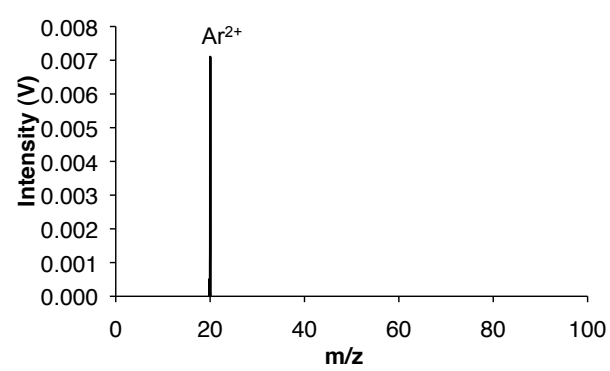

(a)

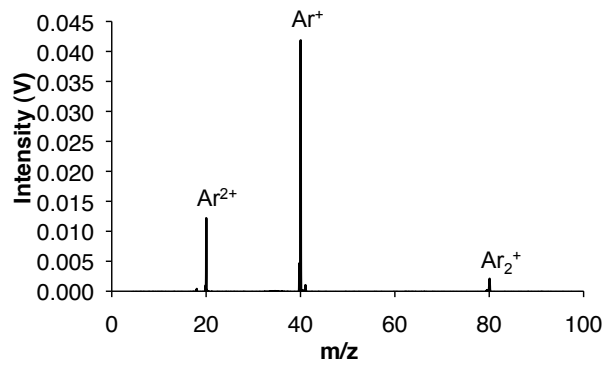

(b)

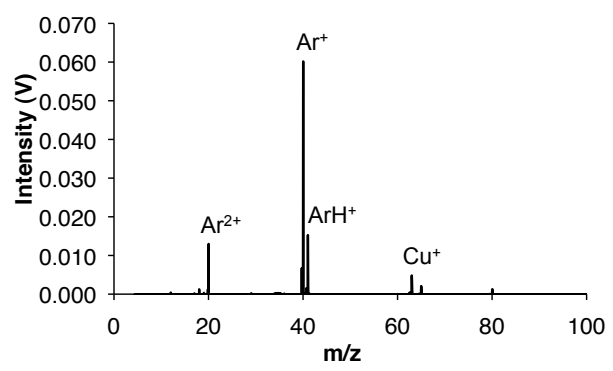

(c)

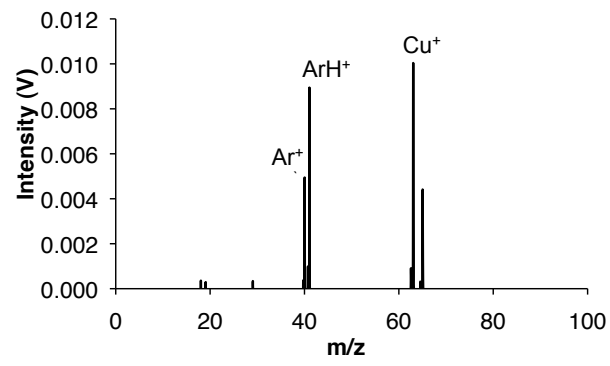

(d)

Figure 2.2: Sample mass spectra from different time regimes of the GD pulse. Repeller delay: (a) $8 \mu \mathrm{s}$, (b) $18 \mu \mathrm{s}$, (c) $3 \mathrm{~ms}$, (d) $5.25 \mathrm{~ms}$. Operating conditions: $5.0 \mathrm{~ms}$ GD pulse width, $50 \%$ duty cycle, $10 \mathrm{~mm}$ cathode distance, $2.5 \mathrm{~W}$ instantaneous GD pulse power, 0.60 Torr argon pressure. 
the pulse cycle for a $2.5 \mathrm{~ms}, 5.0 \mathrm{~ms}$, and $7.5 \mathrm{~ms}$ pulse respectively. Data collected from the oscilloscope was processed using a LabVIEW program that plotted the absolute peak intensity for the ion of interest of each mass spectrum versus the repeller delay time yielding an ion intensity profile.

\subsection{Results and discussion}

\subsubsection{Temporal profiles of gas and sputtered species}

The steady state dc glow discharge produces a stable source of ions for analysis. In contrast, pulsed glow discharges exhibit reproducible temporal profiles of ion signals that do not match the applied square-wave voltage pulse. This is because pulsed glow discharges, unlike their steady-state counterpart, have three main regions: (1) a transitional period in which the power is first initiated, (2) a steady-state period in which the power has stabilized, and (3) a second transitional period just following power termination.

It is well known that the ion signal from the discharge gas exhibits an intensity maximum (prepeak) immediately following the application of discharge power and the ion signal from the sputtered cathode material rapidly increases to a maximum (afterpeak) upon cessation of the applied power. Figure 2.3 shows a typical voltage pulse and ion temporal profiles for gas and sputtered species for a $5 \mathrm{~ms}$ pulsed glow discharge operated with a frequency of $100 \mathrm{~Hz}$. The applied voltage of the dc power supply was measured using a high voltage probe and digital oscilloscope. The leading edge of the voltage pulse spikes and then levels off within $0.5 \mathrm{~ms}$ of power application. "Ringing" in the waveform when the pulse terminates creates some observed instabilities for $\mathrm{Ar}^{+}$in the signal around $5.02 \mathrm{~ms}$. The following observations are based on a pulsed glow discharge used for analytical measurements operating with a $100 \mathrm{~Hz}, 5$ millisecond discharge pulse with copper for the cathode material and argon as the discharge gas.

\subsubsection{Effects of spatial sampling on ion temporal profiles}

The position of the ion exit orifice with respect to the glow discharge plasma is very important because of the relative ionization regions that can be sampled. The mass analyzer samples ions from the discharge chamber via this orifice. Varying the distance between the ion exit orifice and the cathode thus changes the region of 


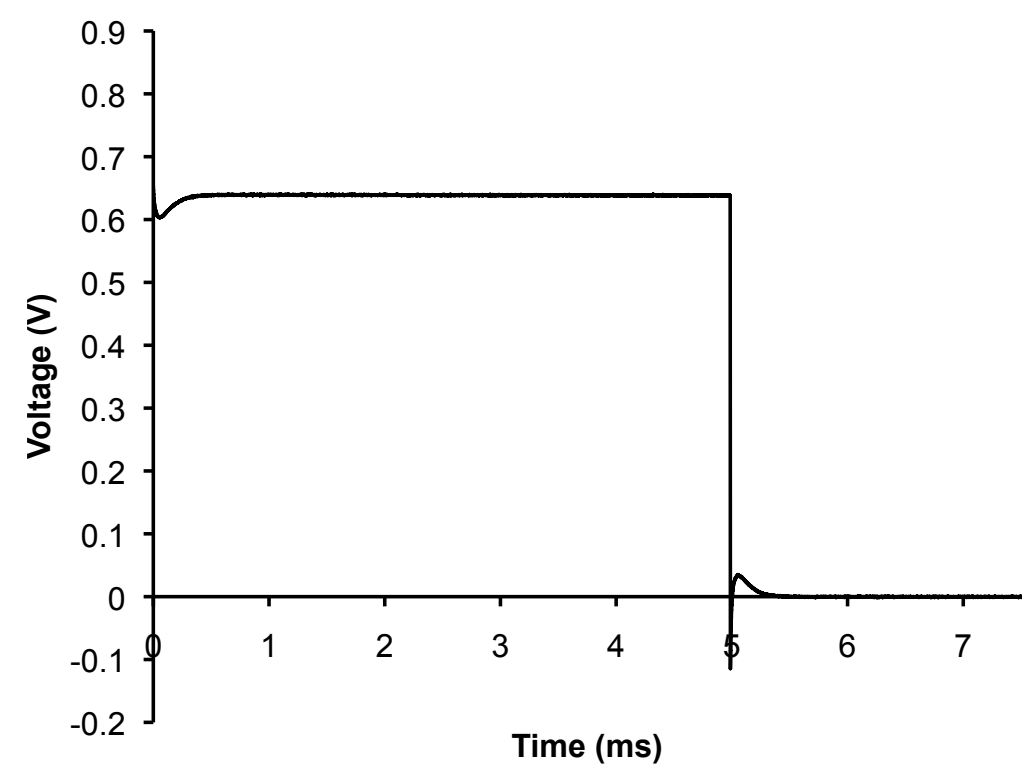

(a)

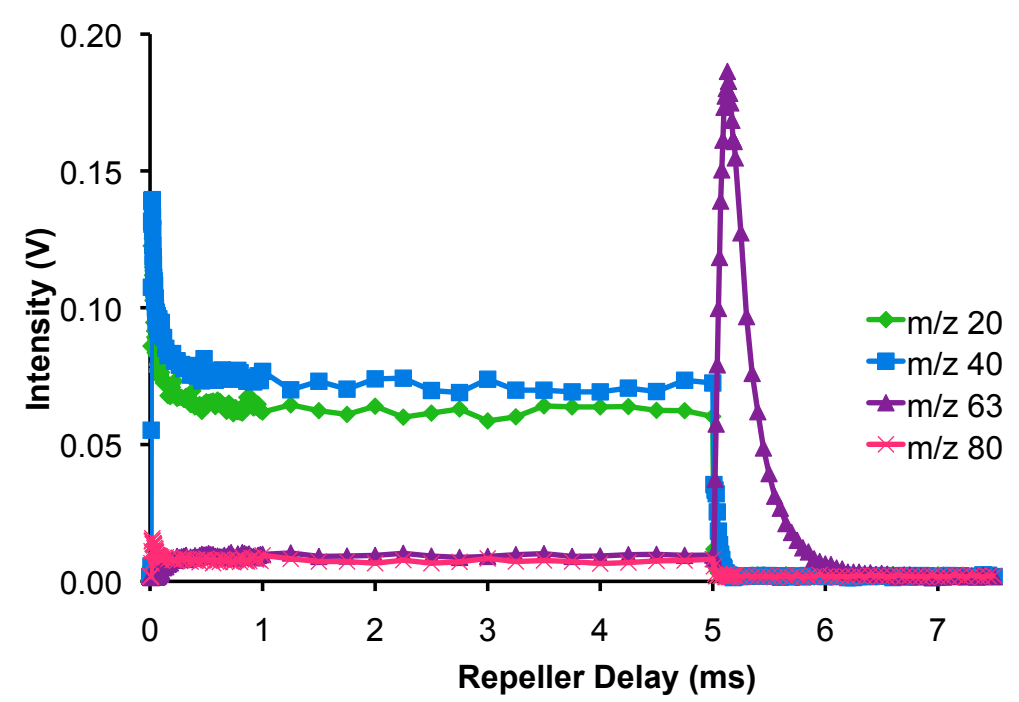

(b)

Figure 2.3: Pulsed voltage waveform and typical ion temporal profiles observed in a pulsed argon GD: (a) Applied dc voltage waveform for a $5 \mathrm{~ms}$ dc pulse; (b) temporal profiles for gas species: ${ }^{40} \mathrm{Ar}^{2+}$ (m/z 20), ${ }^{40} \mathrm{Ar}^{+}$(m/z 40), ${ }^{40} \mathrm{Ar}_{2}^{+}$(m/z 80) and sputtered species: ${ }^{63} \mathrm{Cu}^{+}(\mathrm{m} / \mathrm{z}$ 63). Operating conditions: $5.0 \mathrm{~ms}$ GD pulse width, $50 \%$ duty cycle, $4 \mathrm{~mm}$ cathode distance, $1.0 \mathrm{~W}$ instantaneous GD pulse power, 0.60 Torr argon pressure. 
the plasma sampled. $\frac{15116}{1}$ This is important as atomization and excitation/ionization processes vary in distinct regions of the discharge and most of the ions sampled by the mass spectrometer are those that have been ionized in the locality of the orifice. 17 Figure 2.4 illustrates the effects of varying the cathode to ion exit orifice distance from 2 to $12 \mathrm{~mm}$ with power, pressure, and pulse width held constant.

In order to identify the factors influencing formation of the prepeak and plateau intensities of discharge gas species, the distance from cathode to ion exit orifice was altered by moving the direct insertion probe. In Figures 2.4a, 2.4b, and 2.4c, it was observed for our discharge gas ions, ${ }^{40} \mathrm{Ar}^{2+},{ }^{40} \mathrm{Ar}^{+}$, and ${ }^{40} \mathrm{Ar}_{2}^{+}$, that their prepeaks and plateaus were at a maximum near the cathode surface and then decreased with increasing cathode to ion exit orifice distances. Argon ion populations are formed predominantly through electron ionization of gas atoms near the cathode. Electrons accelerating away from the negatively charged cathode have an energy of about 25 $\mathrm{eV}$.11 After traversing the cathode dark space (CDS), these electrons experience a decline in electrostatic repulsion and are further subjected to collisions, both resulting in a loss of kinetic energy of approximately $7 \mathrm{eV} \stackrel{11}{11}$ A large electron density exists at the anode edge of the CDS due to greater levels of ionization. 18 Thus, the most energetic and highest density of electrons are found near the cathode surface increasing the prepeak and plateau intensities as the cathode is moved closer to the ion exit orifice.

Copper ions are not observed during the prepeak time period because the cathode material has yet to be sputtered and so no cathode atoms are available for ionization. In contrast to the plateau intensities of argon species, copper ions maximize further from the cathode surface and exhibit a bell-shaped response to changes in distance, Figure 2.4d. Previous reports ${ }^{15 / 19}$ showed that the spatial positioning of the copper ion is independent of the copper atom and instead related to the position of the ionizing agent or changes in excitation processes. During the plateau, copper ions are known to form primarily through a mix of electron ionization, charge exchange, and Penning ionization. With electron populations maximizing just beyond the CDSNG interface and metastable argon atom populations maximizing at distances further into the NG, Figure 2.4d supports the dependence of copper ions on metastable argon atoms during not only the afterpeak, $\frac{20}{20}$ but the plateau as well.

Using atomic spectroscopy, it has been shown that the majority of the metastable argon atom population, responsible for Penning ionization, resides adjacent to the cathode surface during steady-state (i.e. plateau) conditions. 1612122 Although the maximum metastable argon atom density exists at the cathode surface during steadystate conditions, with a steep decline throughout the cathode dark space, a second 


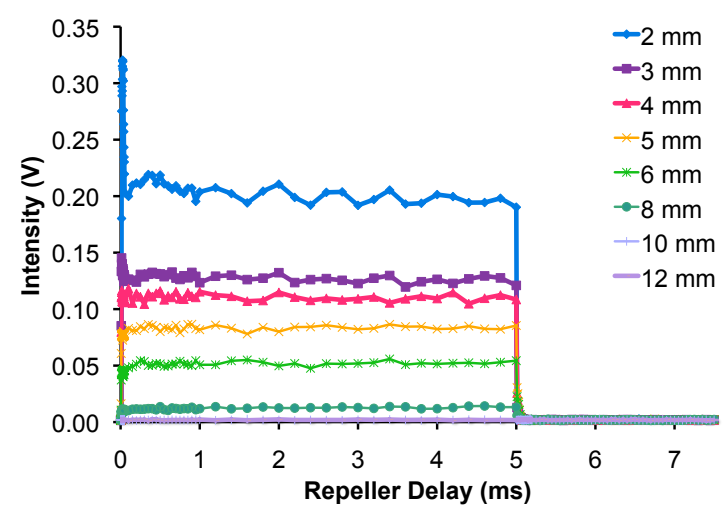

(a) ${ }^{40} \mathrm{Ar}^{2+}$

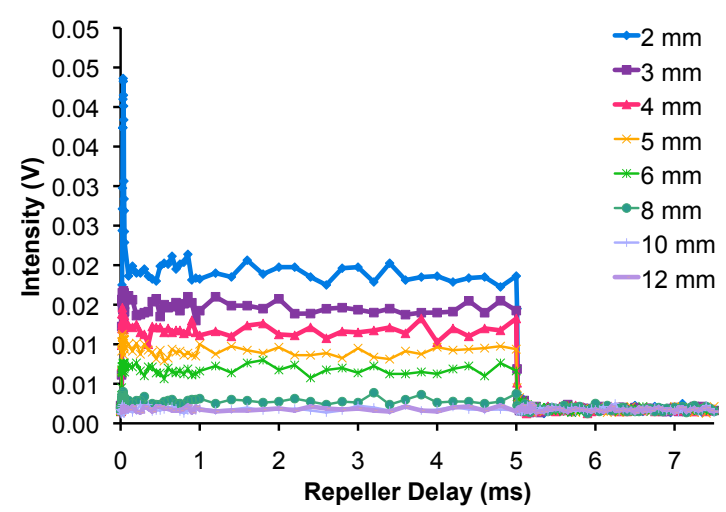

(c) ${ }^{40} \mathrm{Ar}_{2}^{+}$

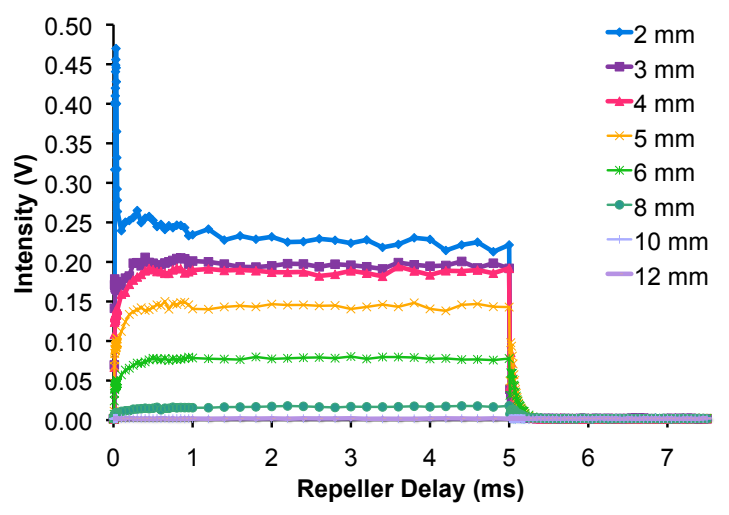

(b) ${ }^{40} \mathrm{Ar}^{+}$

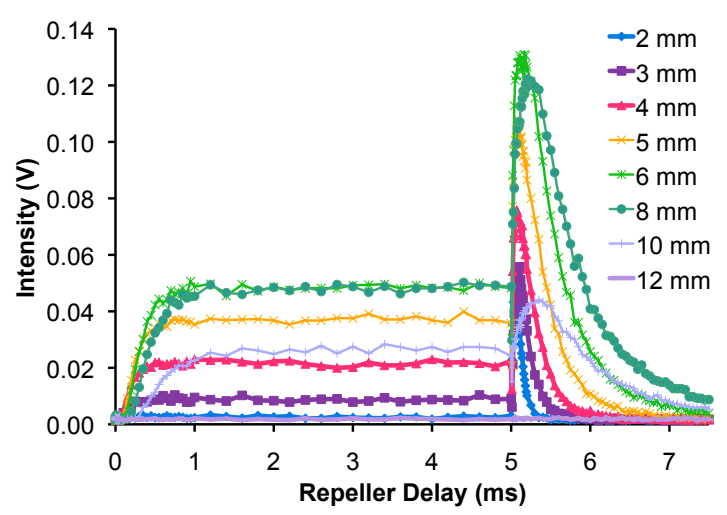

(d) ${ }^{63} \mathrm{Cu}^{+}$

Figure 2.4: Effect of distance from cathode to ion exit orifice on ion intensity profiles of ${ }^{40} \mathrm{Ar}^{2+},{ }^{40} \mathrm{Ar}^{+},{ }^{40} \mathrm{Ar}_{2}^{+}$, and ${ }^{63} \mathrm{Cu}^{+}$. Operating conditions: $5.0 \mathrm{~ms}$ GD pulse width, $50 \%$ duty cycle, $1.0 \mathrm{~W}$ instantaneous GD pulse power, 0.60 Torr argon pressure. 
maximum is present well within the negative glow. 21 It was suggested by Jackson $\frac{16}{16}$ and Lewis ${ }^{22}$ that the metastable argon atom population maximum found near the cathode is unavailable for Penning ionization most likely due to rapid de-excitation by the large electron population residing in the same area. This leaves the second maximum of metastable argon atoms located well within the negative glow responsible for Penning ionization and the population of sputtered ions. As seen in Figure $2.4 \mathrm{~d}$, the copper ion population follows the general shape and location of this second maximum during the plateau period; a broad peak in ion intensity is observed between a 2 to $12 \mathrm{~mm}$ separation of cathode and anode. After pulse termination (i.e. during the afterpeak), Jackson et al. ${ }^{[16}$ observed the bulk of the metastable argon atom population to move away from the cathode to a distance of 4-8 $\mathrm{mm}$ - in excellent agreement with the maximum mass spectrometric measurement of copper ions shown here.

During their journey from cathode to anode, electrons undergo elastic collisions promoting recombination with argon atoms to form metastable argon atoms. Electrons must go through a number of these collisions before thermalizing, creating the upward trend in copper ion intensity as the glow discharge is sampled near the cathode surface on outward. A maximum is seen at intermediate distances where electron thermalization and recombination is at its peak. Moving further from this point and further from the cathode, argon metastables are subjected to an increasing number of de-excitation collisions and a downward trend in copper ion intensity is apparent.

In Figure 2.4d, it is observed that increases in the cathode to ion exit orifice distance shift the onset of the copper plateau further in time with respect to the initiation of the discharge pulse. This temporal shift reflects the increased time required for the sputtered cathodic atoms to diffuse to the ion exit orifice as the space over which the ions must drift is increased. On the other hand, the sampling distance has little or no effect on the appearance of plateau signals for the discharge gas ion, argon, Figures 2.4a, 2.4b, and 2.4c. Argon atoms are not confined to the area surrounding the cathode and thus can be found near the ion exit orifice upon discharge initiation. Similar results were reported in diffusion studies by Hang et al. for a pulsed glow discharge operating on the microsecond time scale. $17 / 23$

Figure $2.4 \mathrm{~d}$ also illustrates the effect of increased sampling distance on the temporal shape and position of copper's afterpeak. With changes in sampling distance a noticeable change in the shape of the afterpeak is observed. At shorter distances the afterpeak of copper demonstrates a sharp increase in intensity and decay down to baseline intensities. As the sampling distance is increased, the afterpeak maximum shifts to a different temporal location and becomes temporally broader. It also 
gradually rises to its maximum intensity and then gradually decays as a function of sampling distance. Sputtered copper atoms must first drift across the discharge chamber towards the ion exit orifice and then be ionized within its vicinity. The sputtered atoms must diffuse farther to the orifice when the cathode-orifice distance is increased. This changing of distance significantly affects the afterpeak shape and placement of the maximum intensity for the sputtered-species copper by increasing the total time it takes the ions to reach the detector. For this reason, figures in this report plotting the afterpeak intensity were constructed by measuring the maximum afterpeak intensity at different time delays according to the cathode to ion exit orifice distances.

\subsubsection{Effects of power and pressure on ion temporal profiles and intensity}

\subsubsection{Power.}

Operation of the glow discharge at a constant power provides greater signal stability than operation in either a constant voltage or constant current operation. Temporal profiles for ${ }^{40} \mathrm{Ar}^{2+},{ }^{40} \mathrm{Ar}^{+},{ }^{40} \mathrm{Ar}_{2}^{+}$, and ${ }^{63} \mathrm{Cu}^{+}$were recorded as power was adjusted to $1.0,1.5$, and $2.0 \mathrm{~W}$. The highest power was primarily limited by the stability of the glow discharge.

It was observed that the signal intensities for all four ions varied positively in proportion to power; with higher powers comes increased atomization and ionization. An additional change observed in these profiles resulting from the increase in power was an increase in the afterpeak-to-plateau ion signal ratio for ${ }^{63} \mathrm{Cu}^{+}$, Figure 2.5 . The increase in copper ion signal arises in part from the increase in sputtering arising from the increase in power. In addition, as current increases the argon metastable population is known to increase, ${ }^{24}$ resulting in enhanced Penning ionization of $\mathrm{Cu}$ atoms.

\subsubsection{Pressure.}

Measurements of the mass spectral ion signals of ${ }^{40} \mathrm{Ar}^{2+},{ }^{40} \mathrm{Ar}^{+},{ }^{40} \mathrm{Ar}_{2}^{+}$, and ${ }^{63} \mathrm{Cu}^{+}$ as a function of distance from cathode to anode were made at pressures of 0.30 Torr, 0.60 Torr, and 0.90 Torr in an argon GD plasma. At lower pressures, glow discharges are characterized by longer mean free paths and higher over-all voltages producing 


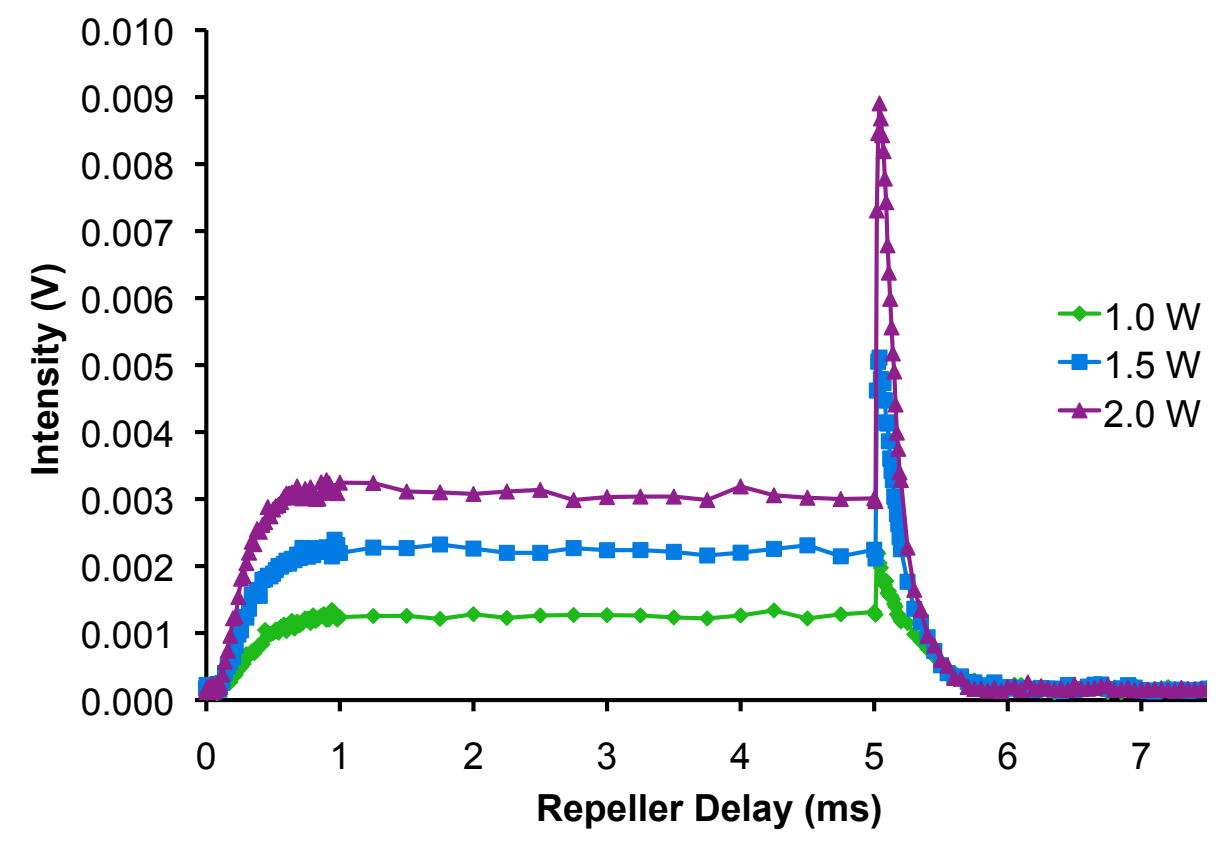

Figure 2.5: Effect of instantaneous GD pulse power on ${ }^{63} \mathrm{Cu}^{+}$intensity profile. Operating conditions: $5.0 \mathrm{~ms}$ GD pulse width, $50 \%$ duty cycle, $6 \mathrm{~mm}$ cathode distance, 0.60 Torr argon pressure. 
ions of higher energy. As the pressure increases, there are more charge carriers, the current increases, thus the voltage must decrease to maintain constant power.

In Figure 2.6, the intensity distributions of the prepeak, plateau, and afterpeak for the ions of interest shifted to increasingly shorter distances as the pressure was increased, indicating a less diffuse and more compressed plasma. It is known that the discharge pressure determines the mean free path, through the density, and thus the collisional frequency. The mean free path, defined as the average distance between collisions, is given by the following equation.

$$
\text { Mean Free Path }=\frac{1}{\pi d^{2} n_{v}}
$$

Where $d$ is the effective diameter of the atom or molecule, $\pi \mathrm{d}^{2}$ is the collision cross-section, and $n_{v}$ is the number of molecules per unit volume. An increase in pressure is accompanied by a decrease in the mean free path of particles. This can be seen visually as a compression of the dark space $e^{\sqrt{25}}$ and a contraction of the negative glow volume. ${ }^{3}$ And as seen in Figure 2.6. mass spectrometric measurements also reveal the negative glow region spans smaller distances and is forced toward the cathode as the GD chamber is increasingly filled with discharge gas. 


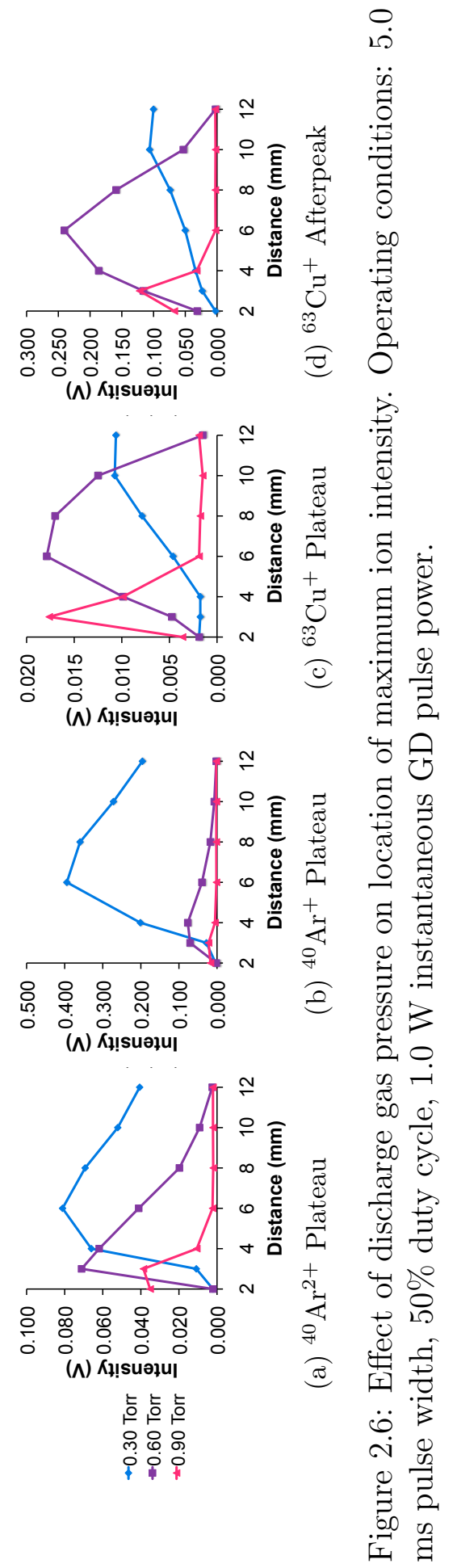


Furthermore, it was observed that as the discharge gas pressure was increased from 0.30 to 0.60 to 0.90 Torr, the maximum intensities of our discharge gas species from the prepeak and plateau periods decreased. This can be explained by an increase in collision frequency. As the pressure, and therefore the gas number density, is increased electrons are subjected to more collisions resulting in a loss of kinetic energy and a smaller population of primary electrons (those released from the cathode) that are capable of electron ionization reaching the ion exit orifice. Additionally, at the lowest pressure, 0.30 Torr, the power is characterized by a comparatively high voltage and low current. This high voltage translates into electrons with high kinetic energies and enhanced electron ionization to form ions of the argon species.

At the three selected pressures, the ${ }^{63} \mathrm{Cu}^{+}$ion profiles in Figure 2.7 reveal changes in intensity. At the lowest pressure examined, 0.30 Torr, the ion temporal profile for ${ }^{63} \mathrm{Cu}^{+}$revealed an intense afterpeak signal reaching about nine times the intensity of its plateau value. As the pressure was increased to 0.60 Torr, the afterpeak exhibited a significant increase in signal indicating the importance of pressure for analytical studies. With a further increase in pressure to 0.90 Torr, the afterpeak is reduced. These observations are in agreement with Smith and co-workers findings that at 0.5-0.6 Torr Penning ionization efficiency is nearly maximized ${ }^{24}$ Furthermore, a previous study ${ }^{\sqrt{3}}$ performed at constant current also found the afterpeak to decrease with an increase in pressure from 0.55 to 1.0 Torr. The decrease in the afterpeak to plateau ratio can be attributed to collisional de-excitation of the metastable argon atom population because increases in pressure increase the probability of de-exciation through collisions with neutral argon support gas. ${ }^{[26}$ An overall decrease in copper ion intensity can be seen at higher pressures because the power is characterized by a comparatively low voltage, such that the kinetic energy of the species impacting the cathode may not be of sufficient energy for atomization and result in low sputter yield.

The population of metastable atoms is a balance between formation processes and destruction processes. ${ }^{27}$ Because the gas-phase species are in continual motion, they are frequently colliding with other species and with the walls. At low pressures the destruction of metastable atoms occurs primarily through diffusion to the walls. ${ }^{28}$ As the pressure is increased, the cross section for the recombination of argon ions with low-energy electrons to form metastable atoms increases and an increase in the copper afterpeak is observed. At further increases in pressure, de-excitation of the metastable atom by two-body or three-body collisions plays a larger role ${ }^{28}$ thus reducing the copper afterpeak. In addition, as pressure increases the amount of redeposition of the sputtered material also increases. It has been estimated that up 


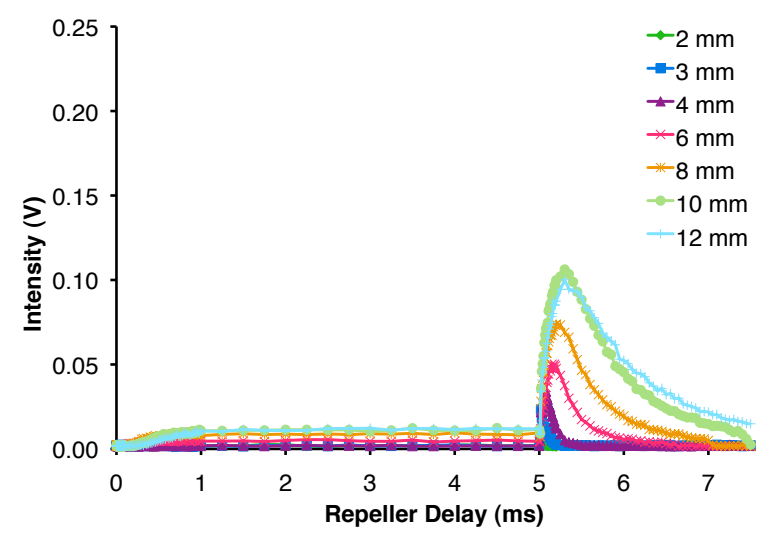

(a) 0.30 Torr

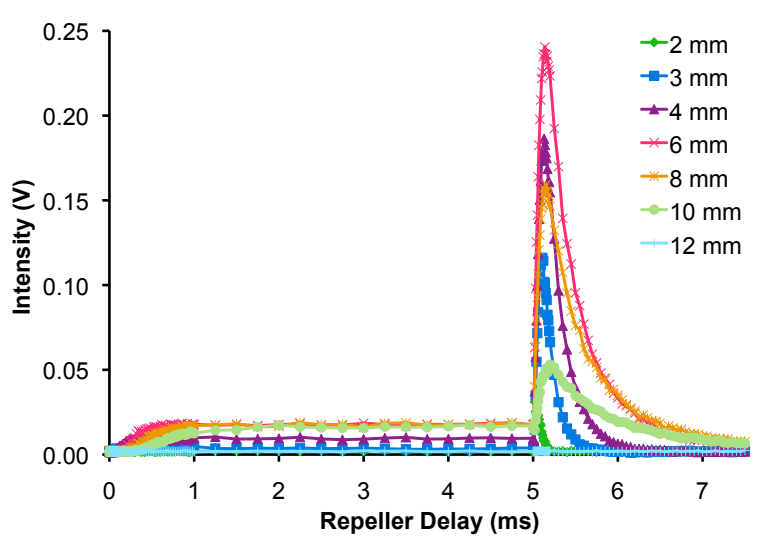

(b) 0.60 Torr

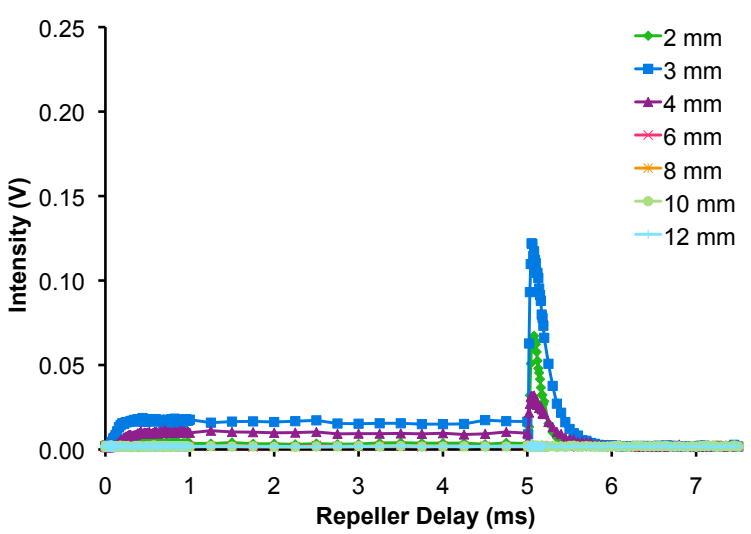

(c) 0.90 Torr

Figure 2.7: Effect of discharge gas pressure on the ion profile of ${ }^{63} \mathrm{Cu}^{+}$. Operating conditions: $5.0 \mathrm{~ms}$ GD pulse width, $50 \%$ duty cycle, $1.0 \mathrm{~W}$ instantaneous GD pulse power, maximum copper signal measured at $10 \mathrm{~mm}$ for 0.30 Torr, $6 \mathrm{~mm}$ for 0.60 Torr, and $3 \mathrm{~mm}$ for 0.90 Torr. 
to $90 \%$ of the sputtered material is redeposited at 1.0 Torr, ${ }^{29}$ with more diffusion and less redeposition at lower pressures.

The shapes of the ${ }^{63} \mathrm{Cu}^{+}$profiles are also affected by changes in pressure when distance is held constant, Figure 2.8. Intensities were normalized to maximum plateau values for discharge initiation and maximum afterpeak values for discharge termination. Because the plasma compresses or expands with pressure, a single sampling distance at which all three pressures produced a measurable signal was not found. As seen in Figure 2.8a, following discharge initiation, ${ }^{63} \mathrm{Cu}^{+}$ions take longer to reach their plateau values; and as seen in Figure 2.8b, following discharge termination, the width of the afterpeak grows. When distance is held constant and pressure is increased, the arrival time of sputtered ions at the detector increases because it takes longer for them to diffuse from cathode to ion exit orifice. Although microsecond GD pulses do not exhibit a typical plateau and afterpeak, the results shown here compare favorably with a microsecond GD pulse study by Hang and Harrison. ${ }^{17}$ In Figure 2.7 it appears that the opposite is true; increased pressure decreases arrival time and shrinks the width of the afterpeak. But the aforementioned effects of distance must also be considered. This indicates that the sputtered atom diffusion time is affected more strongly by variations in distance from cathode to ion exit orifice than by variations in pressure.

\subsubsection{Effects of pulse width and duty cycle with constant frequency on ion temporal profiles and ion intensity}

GD pulse width and duty cycle are critical parameters for pulsed operation. Efimova, et. al. ${ }^{30}$ have noted this for the pulsed Grimm glow discharge. These parameters both have a similar effect on the ionization processes during and following the pulse. In this study a constant frequency of $100 \mathrm{~Hz}$ was used with pulse widths of 2.5, 5.0, and $7.5 \mathrm{~ms}$ at duty cycles of $25 \%, 50 \%$ and $75 \%$ respectively. The focus was to examine how these changes impacted the ion signals while constant power was maintained during the plasma pulse and not as an average over the entire pulse cycle. The findings are consistent with those reported previously for the Grimm glow discharge. 


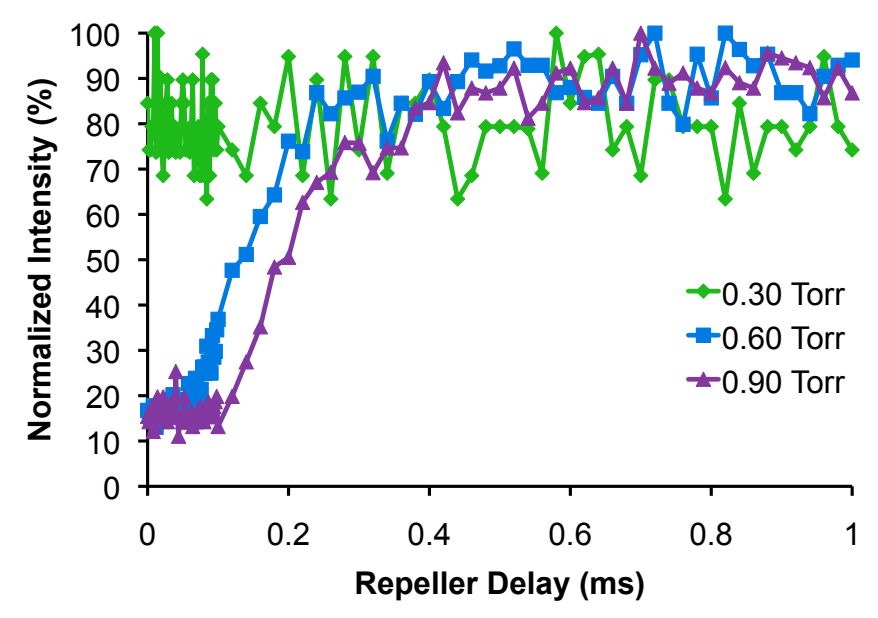

(a) Discharge initiation; $4 \mathrm{~mm}$

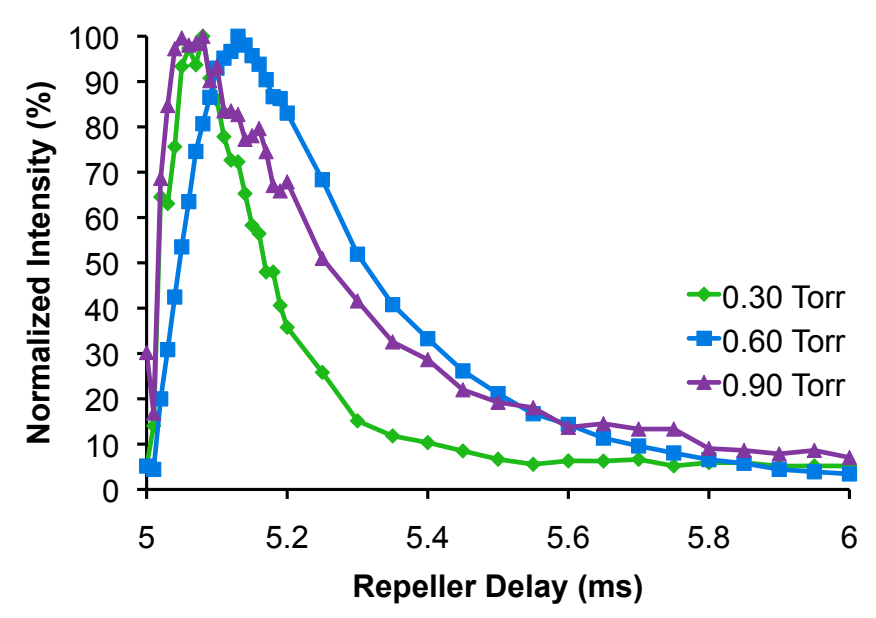

(b) Discharge termination; $4 \mathrm{~mm}$

Figure 2.8: Effect of discharge gas pressure on arrival time of sputtered species, ${ }^{63} \mathrm{Cu}^{+}$. Operating conditions: $5.0 \mathrm{~ms}$ GD pulse width, $50 \%$ duty cycle, $4 \mathrm{~mm}$ cathode distance, $1.0 \mathrm{~W}$ instantaneous GD pulse power. 


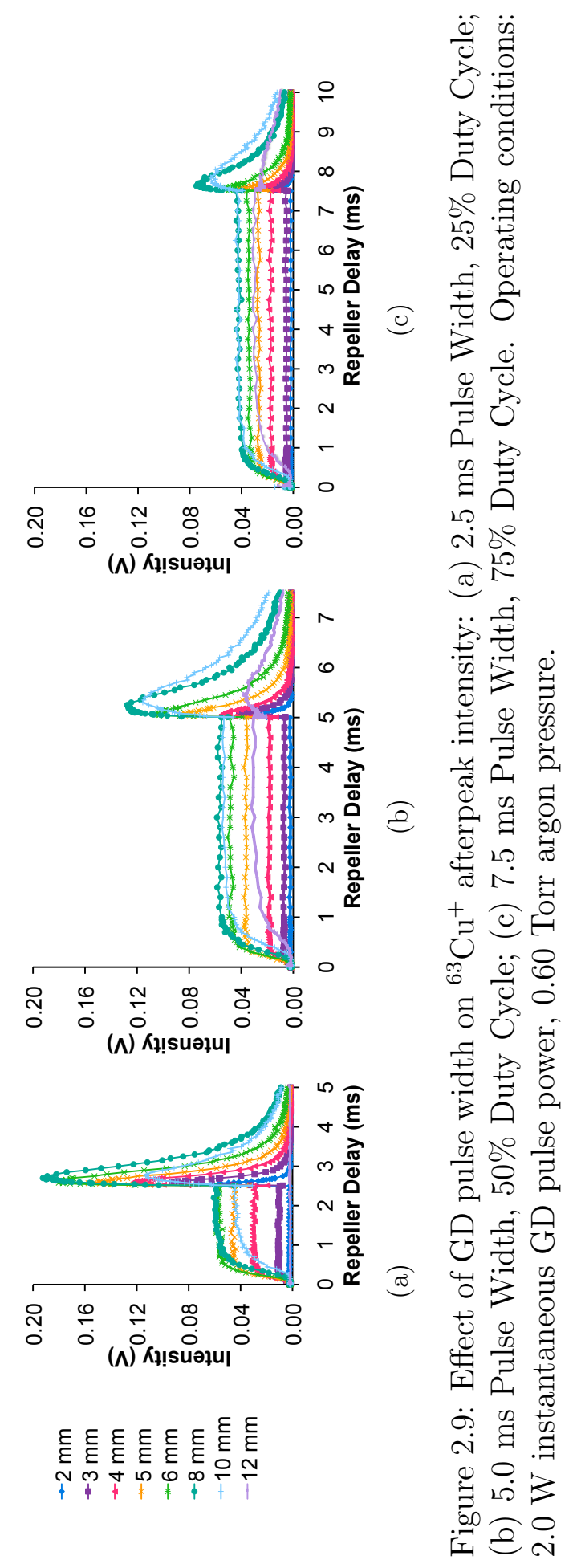


The cathode distance at which the plateau and afterpeak values maximized remained unaffected by changes in pulse width and duty cycle. For example, all three maximized at $8 \mathrm{~mm}$ for ${ }^{63} \mathrm{Cu}^{+}$as seen in Figure 2.9. Plateau ion intensities for our discharge gas ions, ${ }^{40} \mathrm{Ar}^{2+},{ }^{40} \mathrm{Ar}^{+}$, and ${ }^{40} \mathrm{Ar}_{2}^{+}$, and sputtered ion, ${ }^{63} \mathrm{Cu}^{+}$, are not largely affected by changes in pulse width and duty cycle.

Although the effect on plateau ${ }^{63} \mathrm{Cu}^{+}$intensity is minimal, there is a slight decrease in afterpeak ${ }^{63} \mathrm{Cu}^{+}$intensity with increased duty cycle and increased pulse width. Moving to shorter pulse widths and smaller duty cycles, ${ }^{63} \mathrm{Cu}^{+}$experienced a small increase in intensity during the plateau and a sizable increase during the afterpeak, Figure 2.10. Presumably, with the application of constant power pulses for all three pulse widths, the sputtered atom population should remain unchanged. And when the power terminates, the same number of sputtered atoms is available for Penning ionization. Yet the afterpeak of $\mathrm{Cu}$ is clearly greatest at the shortest pulse width, and smallest duty cycle, examined. With the sputtered atom population constant, changes in the argon metastable population appear to be the cause. This suggests that longer plateaus minimize the production of argon metastables in the afterpeak. This could be explained by an increased population of higher energy argon atomic states that would be less likely to form metastable argon atoms.

This study shows that when applied power is constant, decreases in the pulse width and duty cycle have no obvious effect on the intensities of discharge gas ions, whereas, the sputtered ion is positively affected. These findings highlight the importance of pulse width and duty cycle in optimizing sputtered ion signals over those of discharge gas species. These two parameters are the focus of future work for insight into plasma behavior.

\subsubsection{Doubly charged gas ions}

For a steady-state analytical glow discharge mass spectrometer using argon as the discharge gas, the generation ratio of $\mathrm{Ar}^{+}$to $\mathrm{Ar}^{2+}$ has been reported to be about 100:20 by Harrison ${ }^{31}$ and 100:10 by Itoh et al. ${ }^{[32}$ For a pulsed glow discharge, an interesting reproducible phenomenon occurs within the initial moments of the glow discharge pulse. At the start of the pulse cycle, the first ions to be detected are of doubly charged argon at $8 \mu \mathrm{s}$, Figure 3.5a. Between their first appearance and roughly $12 \mu \mathrm{s}$, depending on discharge conditions, an unusual situation exists where doubly charged argon with an ionization potential of $27.6 \mathrm{eV}$ is in greater intensity than singly charged argon with an ionization potential of $15.8 \mathrm{eV}$. The only significant source of ionization during this time period is electron ionization. The most probable 


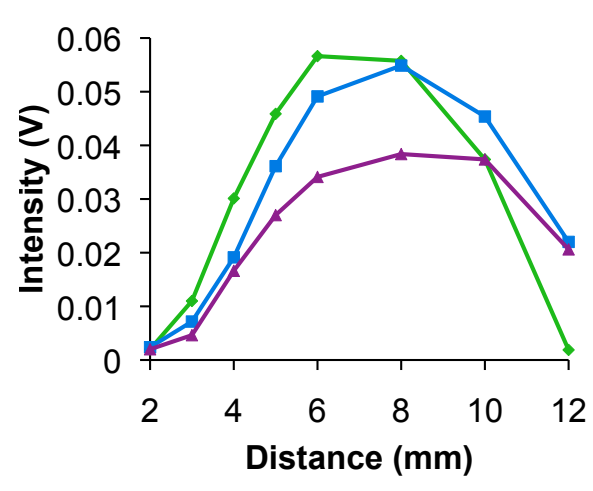

(a) ${ }^{63} \mathrm{Cu}^{+}$during plateau

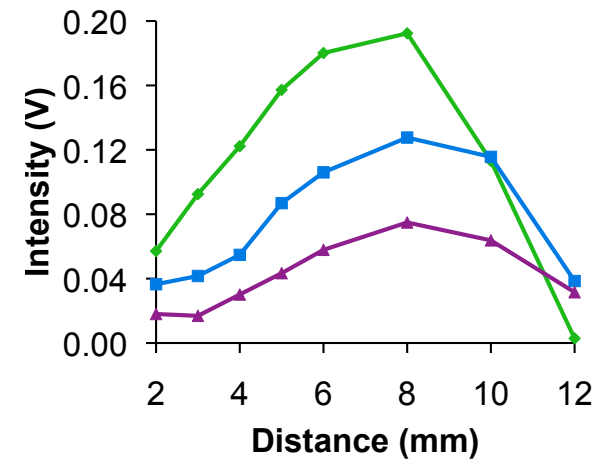

(b) ${ }^{63} \mathrm{Cu}^{+}$during afterpeak

\section{$\rightarrow 2.5$ ms Pulse Width, $25 \%$ Duty Cycle \\ $=5.0 \mathrm{~ms}$ Pulse Width, $50 \%$ Duty Cycle \\ $\Rightarrow 7.5$ ms Pulse Width, $75 \%$ Duty Cycle}

Figure 2.10: Effect of GD pulse width on the plateau and afterpeak intensities of ${ }^{63} \mathrm{Cu}^{+}$. Operating conditions: $2.0 \mathrm{~W}$ instantaneous GD pulse power, 0.60 Torr argon pressure. 
ionization mechanism for the formation of doubly charged gas ions is within a single electron ionization step,

$$
A r+e_{\text {fast }}^{-} \rightarrow A r^{2+}+3 e_{\text {slow }}^{-}
$$

as $\mathrm{Ar}^{2+}$ is detected first; should the appearance of $\mathrm{Ar}^{2+}$ been a result of two electron ionization steps,

$$
\begin{gathered}
A r+e_{\text {fast }}^{-} \rightarrow A r^{+}+2 e_{\text {slow }}^{-} \\
A r^{+}+e_{\text {fast }}^{-} \rightarrow A r^{2+}+2 e_{\text {slow }}^{-}
\end{gathered}
$$

or by the reaction

$$
A r^{+}+A r^{m} \rightarrow A r^{2+}+A r^{0}+e^{-}
$$

its precursor, $\mathrm{Ar}^{+}$, should have been the first detected.

Initially (before the power is applied) the system of argon atoms is in a state of thermal equilibrium. With the application of power, the electrical discharge feeds energy into the argon medium and excites the atoms from the ground state to higher energy levels including continuum (ionization). The system is no longer equilibrated and $\mathrm{Ar}^{2+}$ is observed to dominate the mass spectrum. This observation indicates that during the initial period of discharge breakdown the difference in the temperature between electrons and other discharge particles is even greater than the normal difference observed in the period immediately following discharge breakdown and steady state operation. The population inversion lasts only about $4 \mu \mathrm{s}$, after which the singly charged argon ion surpasses in intensity the doubly charged argon ion for the remainder of the glow discharge pulse. Although atoms are being continuously excited by the electrical discharge during the pulse, the system is able to reach equilibrium through the de-exciting processes of spontaneous and stimulated emission, and collisions with other atoms or molecules.

Distance from cathode to ion exit orifice and pressure of the discharge gas were varied to determine what, if any, effect they have on doubly versus singly charged argon. When distance is varied, the same general trends can be seen for $\mathrm{m} / \mathrm{z} 20$ and $\mathrm{m} / \mathrm{z}$ 40, Figure 2.11. Both ions maximize at the same distance and decrease in intensity with pressure. This agreement indicates that both ${ }^{40} \mathrm{Ar}^{2+}$ and ${ }^{40} \mathrm{Ar}^{+}$ operate under the same mechanism of formation, electron ionization. 


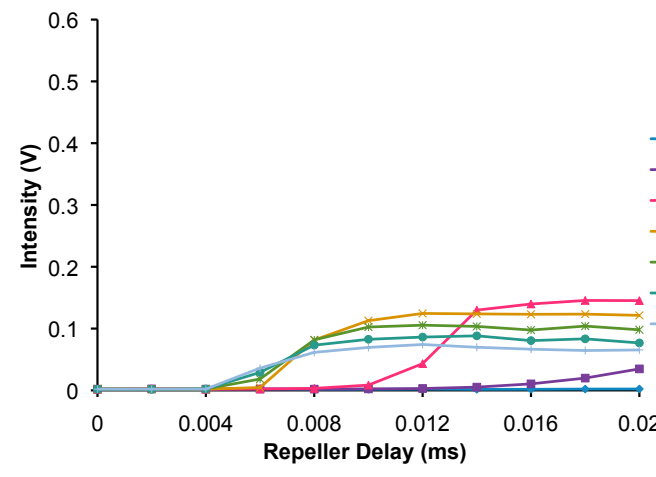

(a) ${ }^{40} \mathrm{Ar}^{2+}$ at 0.30 Torr

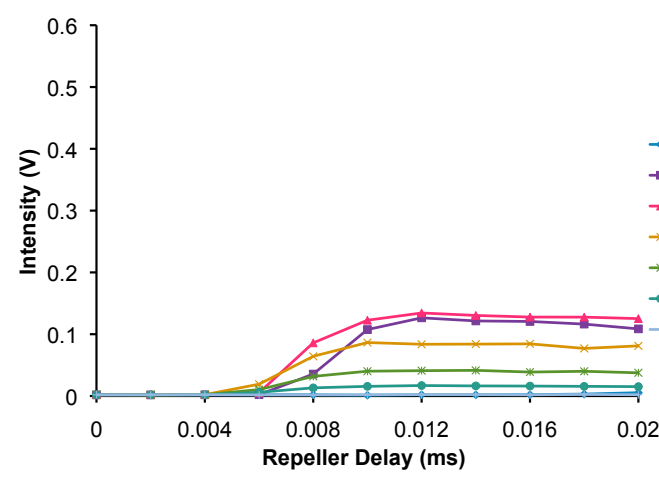

(c) ${ }^{40} \mathrm{Ar}^{2+}$ at 0.60 Torr

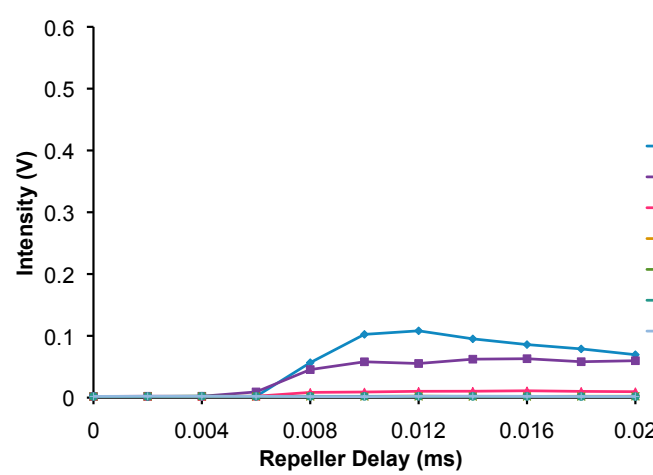

(e) ${ }^{40} \mathrm{Ar}^{2+}$ at 0.90 Torr

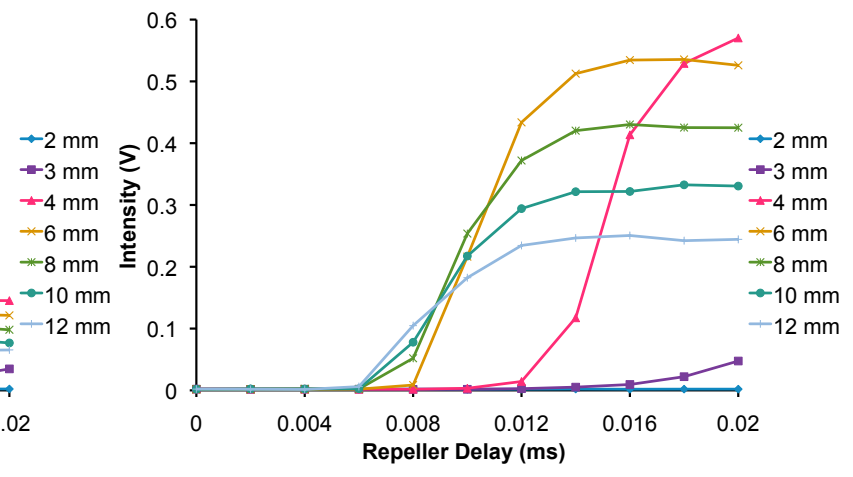

(b) ${ }^{40} \mathrm{Ar}^{+}$at 0.30 Torr

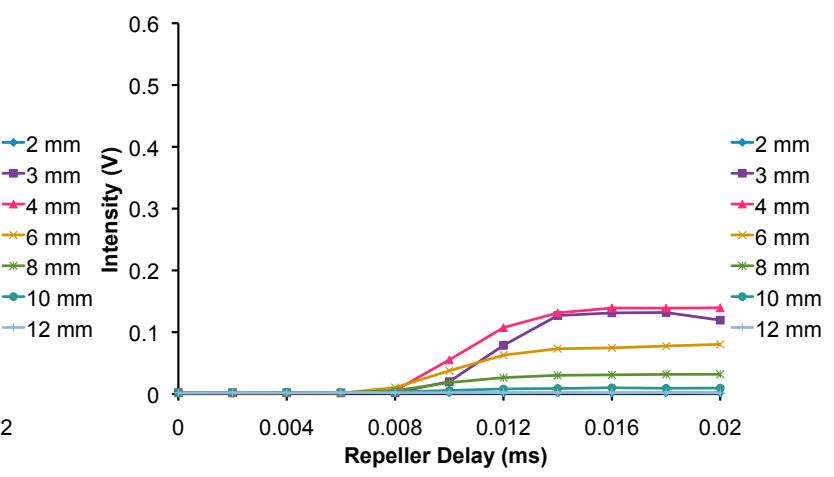

(d) ${ }^{40} \mathrm{Ar}^{+}$at 0.60 Torr

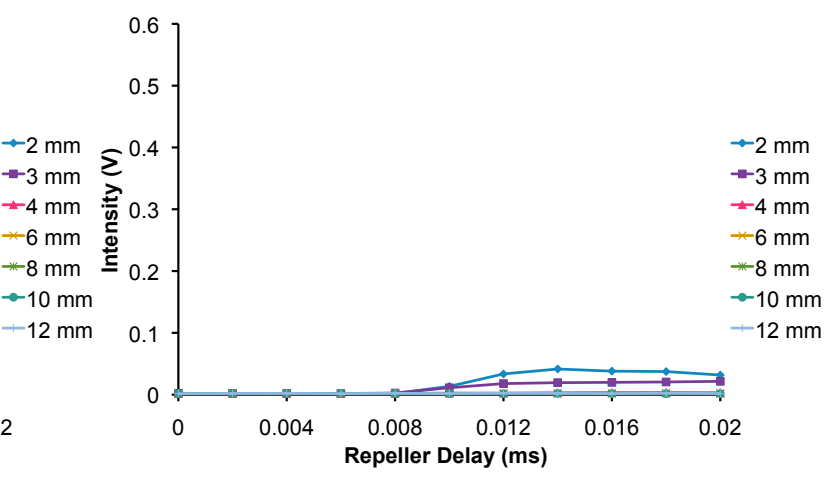

(f) ${ }^{40} \mathrm{Ar}^{+}$at 0.90 Torr

Figure 2.11: Effects of cathode distance and discharge gas pressure on the intensities of ${ }^{40} \mathrm{Ar}^{2+}$ and ${ }^{40} \mathrm{Ar}^{+}$at plasma initiation. Operating conditions: $5.0 \mathrm{~ms}$ GD pulse width, $50 \%$ duty cycle, $1.0 \mathrm{~W}$ instantaneous GD pulse power. 
From Figure 2.11, when pressure is increased it appears that ${ }^{40} \mathrm{Ar}^{2+}$ is favored over ${ }^{40} \mathrm{Ar}^{+}$well past the first few microseconds of plasma initiation. Although at elevated pressures the doubly charged argon ion is more intense than the singly charged argon ion, when the intensities of the $\mathrm{Ar}^{+}$and $\mathrm{ArH}^{+}$are added together, they are well above that of the doubly charged argon ion, $\mathrm{Ar}^{2+}$.

In Figures $2.11 \mathrm{a}$ and $2.11 \mathrm{~b}$, it is interesting to note at 6 or $8 \mu \mathrm{s}$, when the argon ions are first detected, the farthest distance produces the most intense signal, i.e. the farther the distance, the shorter the time in which plateau intensities are reached. These observations indicate that within the first few microseconds of plasma initiation, the plasma has not been fully established and plasma shielding has not yet taken effect. The presence of the electric field retards the argon ions. The closer the ions are to the cathode, the longer is their journey through the exit orifice. Figures $2.11 \mathrm{e}$ and $2.11 \mathrm{f}$ show that as pressure is increased, this transition point becomes sharper and less discernible.

\subsection{Conclusions}

In this work, a de glow discharge established in argon under constant power was examined to determine the effects of certain operating parameters on the plasma's ionization processes and mass spectral ion signals. Through the adjustment of critical parameters such as sampling distance, pulse power, discharge gas pressure, pulse width, and duty cycle the unique temporal pulse profiles of analytically important (sputtered) species from mass spectrometric measurements were acquired.

Ion intensity temporal profiles were constructed from mass spectrometric measurements of a pulsed glow discharge plasma established in argon with a copper cathode. This work expands upon previously reported ion signal profiles by maintaining constant instantaneous power of the glow discharge pulse. The profiles presented here illustrate the differences between ions of discharge gas species $\left({ }^{40} \mathrm{Ar}^{2+},{ }^{40} \mathrm{Ar}^{+},{ }^{40} \mathrm{Ar}_{2}^{+}\right)$, and ions of sputtered atoms $\left({ }^{63} \mathrm{Cu}^{+}\right)$. The sputtered ions and the discharge gas ions are formed during different times of the pulse cycle. The adjustment of conditions can be done to further maximize ion signals from sputtered species while reducing those from discharge gas species. Mass spectrometric measurements indicate that ion signals from analytical species during the afterpeak were further enhanced at intermediate distances between cathode and ion exit orifice $(6-8 \mathrm{~mm})$, high GD pulse power $(2.0 \mathrm{~W})$, intermediate discharge gas pressure (0.60 Torr), shorter pulse widths (2.5 ms), and smaller duty cycles $(25 \%)$. 
When the glow discharge is pulsed, the first ion to be detected is doubly charged argon, indicating an ionization potential of at least $27 \mathrm{eV}$. Discharge gas ions were found to maximize close to the cathode surface because of their dependence upon the location of the electron population. Whereas, ions of the cathode material maximized approximately $6-8 \mathrm{~mm}$ away, indicating the location of metastable argon atoms and the substantial role of Penning ionization over electron ionization of copper atoms. Ion profiles have illustrated that the length of the dark space and of the negative glow varies significantly with discharge gas pressure. As discharge pressure is increased, the dark space and negative glow of the GD spans shorter distances and compresses towards the cathode. Under constant power, intermediate discharge pressures are favorable for the ionization of sputtered copper atoms due to the balance between ionization processes and collisional deactivation. As duty cycle is increased, the equilibration period increases, reducing metastable atoms in the afterpeak. We believe this is due to the populating of argon atom states that do not readily form argon metastable atoms.

The research presented here is useful in understanding the nature of glow discharge ionization processes as well as the selection of pulsed glow discharge experimental parameters and optimization of the plasma's analytical performance. In refining the theory of how the analytical glow discharge operates, it is more likely to improve upon the existing function and applications of this unique ionization source. 


\section{Bibliography}

[1] J. A. Klingler, P. J. Savickas, and W. W. Harrison, "The pulsed glow discharge as an elemental ion source," Journal of the American Society for Mass Spectrometry, vol. 1, no. 2, pp. 138-143, 1990.

[2] J. A. Klingler, C. M. Barshick, and W. W. Harrison, "Factors influencing ion signal profiles in pulsed glow discharge mass spectrometry," Analytical Chemistry, vol. 63, pp. 2571-2576, 1991.

[3] C. Pan and F. L. King, "Ion formation processes in the afterpeak time regime of pulsed glow discharge plasmas," Am Soc Mass Spectrom, vol. 4, pp. 727-732, 1993.

[4] C. Pan and F. L. King, "Time-resolved studies of ionized sputtered atoms in pulsed radio frequency powered glow discharge mass spectrometry," Analytical Chemistry, vol. 65, no. 22, pp. 3187-3193, 1993.

[5] F. L. King and C. Pan, "Temporal signal profiles of analytical species in modulated glow discharge plasmas," Analytical Chemistry, vol. 65, no. 6, pp. 735-739, 1993.

[6] L. Li, J. T. Millay, J. P. Turner, and F. L. King, "Millisecond pulsed radio frequency glow discharge time of flight mass spectrometry: temporal and spatial variations in molecular energetics," American Society for Mass Spectrometry, vol. 15, pp. 87-102, 2004.

[7] A. C. Muniz, J. Pisonero, L. Lobo, C. Gonzalez, N. Bordel, R. Pereiro, A. Tempez, P. C. N. Tuccitto, A. Licciardellod, and A. Sanz-Medel, "Pulsed radiofrequency glow discharge time of flight mass spectrometer for the direct analysis of bulk and thin coated glasses," Journal of Analytical Atomic Spectrometry, vol. 23, no. 9, pp. 1239-1246, 2008.

[8] T. J. Loving and W. W. Harrison, "Simultaneous analysis of an abnormal glow discharge by atomic absorption spectrometry and mass spectrometry," Analytical Chemistry, vol. 55, pp. 1523-1526, 1983.

[9] M. Parker and R. K. Marcus, "Investigation of sample atomization using a power-modulated ratio-frequency glow discharge source," Applied Spectroscopy, vol. 50, no. 3, pp. 366-376, 1996. 
[10] D. Pollmann, K. Ingeneri, and W. W. Harrison, "Comparison of atomization and ionization processes in direct current, radiofrequency and microsecond pulse discharges," Journal of Analytical Atomic Spectrometry, vol. 11, pp. 849-853, 1996.

[11] F. L. King and W. W. Harrison, "Glow discharge mass spectrometry: an introduction to the technique and its utility," Mass Spectrometry Reviews, vol. 9, pp. 285-317, 1990.

[12] B. Chapman, Glow Discharge Processes: Sputtering and Plasma Etching. New York: Wiley, 1980.

[13] W. W. Harrison and W. Hang, "Powering the analytical glow discharge," Fresenius J Anal Chem, vol. 355, pp. 803-807, 1996.

[14] C. G. Bruhn and W. W. Harrison, "Sputter-atomization studies with a glow discharge," Analytical Chemistry, vol. 50, no. 1, pp. 16-21, 1978.

[15] C. L. Lewis, G. P. Jackson, S. K. Doorn, V. Majidi, and F. L. King, "Spectral, spatial and temporal characterization of a millisecond pulsed glow discharge: copper analyte emission and ionization," Spectrochimica Acta Part B, vol. 56, pp. 487-501, 2001.

[16] G. P. Jackson, C. L. Lewis, S. K. Doorn, V. Majidi, and F. L. King, "Spectral, spatial and temporal characteristics of a millisecond pulsed glow discharge: metastable argon atom production," Spectrochimica Acta Part B, vol. 56, pp. 2449-2464, 2001.

[17] W. Hang and W. W. Harrison, "Diffusion, ionization, and sampling processes in the glow discharge source for mass spectrometry," Analytical Chemistry, vol. 69, no. 24, pp. 4957-4963, 1997.

[18] R. K. Marcus, ed., Glow Discharge Spectroscopies. Plenum Press, 1993.

[19] A. Bogaerts, R. D. Guenard, B. W. Smith, J. D. Winefordner, W. W. Harrison, and R. Gijbels, "Three-dimensional density profiles of argon metastable atoms in a direct current glow discharge: experimental study and comparison with calculations," Spectrochimica Acta, vol. 52, pp. 219-229, 1997.

[20] J. A. Strauss, N. P. Ferreira, and H. G. C. Human, "An investigation into the role of metastable argon atoms in the afterglow plasma of a low pressure discharge," Spectrochimica Acta Part B, vol. 37, pp. 947-954, 1982. 
[21] N. P. Ferreira, J. A. Strauss, and H. G. C. Human, "Distribution of metastable argon atoms in the modified Grimm-type electrical discharge," Spectrochimica Acta, Part B: Atomic Spectroscopy, vol. 37B, no. 4, pp. 273-279, 1982.

[22] C. L. Lewis, L. Li, J. T. Millay, S. Downey, J. Warrick, and F. L. King, "Temporal emission characteristics of millisecond pulsed radiofrequency and direct current glow discharges," Journal of Analytical Atomic Spectrometry, vol. 18, pp. 527-532, 2003.

[23] W. Hang, W. O. Walden, and W. W. Harrison, "Microsecond pulsed glow discharge as an analytical spectroscopic source," Analytical Chemistry, vol. 68, no. 7 , pp. 1148-1152, 1996.

[24] R. L. Smith, D. Serxner, and K. R. Hess, "Assessment of the relative role of penning ionization in low-pressure glow discharges," Analytical Chemistry, vol. 61, no. 10, pp. 1103-1108, 1989.

[25] F. M. Penning, Electrical Discharges in Gases. New York: Wiley, 1957.

[26] M. A. Biondi, "Diffusion, de-excitation, and ionization cross sections for metastable atoms," Physical Review, vol. 88, no. 3, pp. 660-665, 1952.

[27] K. A. Hardy and J. W. Sheldon, "Metastable atom density in helium, neon, and argon glow discharges," Journal of Applied Physics, vol. 53, pp. 8532-8536, 1982.

[28] A. V. Phelps and J. P. Molnar, "Lifetimes of metastable states of noble gases," Physical Review, vol. 89, no. 6, pp. 1202-1208, 1953.

[29] E. Nasser, Fundamentals of Gaseous Ionization and Plasma Electronics. WileyInterscience, 1971.

[30] V. Efimova, V. Hoffmanna, and J. Eckert, "Electrical properties of the microsecond pulsed glow discharge in a Grimm-type source: comparison of dc and rf modes," Journal of Analytical Atomic Spectrometry, vol. 26, pp. 784-791, 2011.

[31] W. W. Harrison, Inorganic Mass Spectrometry, ch. 3: Glow Discharge Mass Spectrometry. Wiley-Interscience, 1988.

[32] S. Itoh, R. Hasegawa, and M. Amano, "Formation of ions in glow discharge mass spectrometry ion sources," Materials Transactions, JIM, vol. 41, no. 2, pp. 346-352, 2000. 


\section{Chapter 3}

\section{Application of Pulsed Glow Discharge Chemical Speciation: Chemical Warfare Agent Metabolites}

\subsection{Abstract}

Although the use of nerve agents is prohibited, concerns remain that humans may become exposed to nerve agents during decommissioning, research, and warfare; therefore, the ability to assess human exposure to nerve agents is needed. Gas chromatography coupled with pulsed glow discharge mass spectrometry was investigated for use as a qualitative screening method for nerve agent metabolites in urine. This research strived to expand the future possibilities of the time-gated pulsed glow discharge ion source through the coupling of gas chromatography to achieve chemical speciation of a urinary metabolite specific to the nerve agent sarin. Chemical speciation can be achieved through structural information from the plateau region and molecular ion information from the afterpeak region and both can be acquired simultaneously. The ability of the pulsed glow discharge to acquire both pieces of information gives the analyst a greater degree of confidence in the identification of the compound; no other technique is capable of providing both pieces of information simultaneously. A nerve agent metabolite was spiked into a synthetic urine sample, extracted onto a solid phase microextraction fiber, desorbed and separated in a gas chromatograph, and 
introduced into the plasma of a pulsed glow discharge time-of-flight mass spectrometer. This study demonstrated that with the use of derivatization reagents, nerve agent metabolites can be analyzed by gas chromatography coupled with pulsed glow discharge mass spectrometry to provide characteristic fragments during the plateau. However, the mass spectra obtained for eluting compounds did not yield molecular information and future directions would focus on adjusting conditions to obtain the molecular ion during the afterpeak.

\subsection{Introduction}

Chemical warfare agents (CWA) are toxic chemicals that can be classified into four groups based on their effect on humans: nerve, blister, blood, and incapacitating. Organophosphorous nerve agents are 15 to 100 times more potent than other CWA, like mustard gas, and are chemically related to organophosphate pesticides. In fact, their use as insecticides was the original intent for most of these chemicals. It is their toxicity to humans that led to their eventual designation and use as weaponry.

More than 180 state parties have ratified the Chemical Weapons Convention and agreed not to produce, stockpile, transfer, or use chemical weapons. This agreement has reduced the risk of chemical warfare agent use, but there is still potential for other parties to make use of these weapons against civilian or military targets. Sarin was used in terrorist attacks in Japan by the Aum Shinrikyo cult. In the first attack in 1994, sarin was released in an apartment complex in Matsumoto, causing 7 deaths and 300 casualties. In March 1995, the same cult released sarin in the Tokyo underground transit system causing 12 deaths and thousands to seek medical care. These terrorist attacks show the ease with which a terrorist organization can both manufacture and deploy CWAs. This incident highlights the importance of the continued development of analytical methods for screening physiological samples to identify the specific chemicals and to assess the level of exposure to these deadly agents. In a mass casualty scenario, evaluation of post-CWA exposure of effected individuals in a timely manner is critical for determining whether CWAs have been used, which agents may have been used, the potential scope of the CWA attacks or incidental exposures, and possible treatment options. Although health care providers will treat the symptoms of victims, a more definitive treatment can be provided if the chemical causing the symptoms is known.

Exposure to nerve agents typically occurs via skin contact or inhalation, but can also be ingested or injected. Nerve agents attack the central nervous system 
through the inhibition of the acetylcholinesterase enzyme, just like their insecticide counterparts. This results in accumulation of the neurotransmitter acetylcholine at the nerve endings and continual stimulation of the body's nervous system, thus destroying normal nerve function. Threshold symptoms of those exposed include miosis (pupil constriction), rhinorrhea (runny nose), and airway constriction. Low to moderate exposure causes sweating, nausea, vomiting, and weakness. Lethal amounts of nerve agent cause convulsions, loss of consciousness, apnea, paralysis, and ultimately death.1

Once inside the body, a small amount of the nerve agent's parent compound is covalently bound to enzymes and tissue proteins, while the majority is hydrolyzed to its corresponding phosphonic acid. ${ }^{2}$ Organophosphorous nerve agents rapidly degrade by hydrolysis into alkyl methylphosphonic acids (AMPA). The nerve agents sarin (GB), soman (GD), and VX form the AMPA hydrolysis products isopropyl methylphosphonic acid (IMPA), pinacolyl methylphosphonic acid (PMPA), and ethyl methylphosphonic acid (EMPA), respectively. All three slowly hydrolyze to form methylphosphonic acid (MPA) as a final degradation product. An illustration of the hydrolysis of sarin to form its corresponding phosphonic acids, IMPA and MPA is shown in Figure 3.1. These AMPA metabolites of nerve agents are excreted from the body and can show up quickly in urine, making them a convenient marker for assessing exposure. Sampling urine has its advantages (over blood for example) as its collection is non-invasive and it offers the analytical chemist a relatively clean sample matrix.

Phosphonic acids are significant as they do not occur naturally and their identification would strongly suggest the prior presence of nerve agents. The most common analytical approach for determining human exposure to nerve agents is the mass

Sarin (GB)

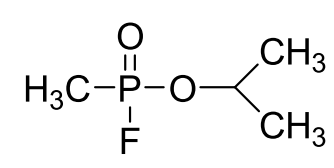

IMPA

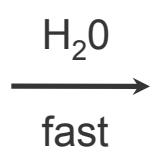<smiles>CC(C)OP(C)(=O)O</smiles>

$\mathrm{C}_{4} \mathrm{H}_{11} \mathrm{O}_{3} \mathrm{P}$

Mol. Wt.: 138.1
MPA

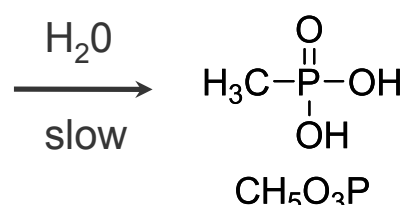

Mol. Wt.: 96

Figure 3.1: Reaction pathway for degradation of sarin to IMPA and MPA. 
spectrometric measurement of the phosphonic acids. Separation, identification, and quantification of these AMPA compounds have been reported using various analytical techniques, with the most popular being liquid chromatography mass spectrometry (LC/MS), ${ }^{3-10}$ liquid chromatography coupled with tandem mass spectrometry (LC/MS/MS), 11 13 gas chromatography mass spectrometry (GC/MS), 14 and gas chromatography coupled with tandem mass spectrometry (GC/MS/MS). ${ }^{20121}$ These methods are capable of analyzing multiple AMPAs in urine at part per billion to part per million levels. Often the use of two or more analytical techniques and authentic reference standards is required for the unambiguous identification of these compounds.

A tunable plasma ion source is capable of providing chemical information ranging from the elemental to the molecular level. One such source is the pulsed glow discharge (GD) employing time-gated detection. Pulsing of the GD offers three distinct time regimes, the prepeak, plateau, and afterpeak, from which elemental, structural, and molecular mass information can be detected. To this end, a complete picture of the analyte molecule, assisting with identification, can be obtained in one analysis. Owing to its unique capabilities, the chemical speciation of samples by pulsed GD-MS has developed into an important method. 22123

Although GD-MS has been applied primarily to the analysis of solid samples, the coupling of chromatographic separations to GD-MS is of particular interest. In 1996, Olson, Belkin, and Caruso ${ }^{24}$ separated tetraalkyltins by GC and detected them by GDMS using helium as both the GC carrier gas and GD plasma gas. A year later, the same group ${ }^{25}$ published work on organotin compounds. The group improved their instrument design by using axial sample introduction for analytes entering the GD plasma as it possibly eliminated discharge asymmetry and increased analyte residence time in the plasma. Both studies used an rf-GD operated in continuous power mode, i.e. not pulsed. Ionization was therefore a result of a mixture of Penning and electron ionization. In 2000, Majidi et al. $\frac{23}{23}$ used time-gated acquisition of a pulsed GD ion source to demonstrate the utility of the pulsed GD approach in the complete elucidation of chemical structure and identity of volatile organic compounds. In this investigation, reagents were leaked into the plasma using a needle valve, often leading to chaotic plasma behavior because of large analyte vapor concentrations. In 2003, Lewis et al. ${ }^{26}$ reported the first real-time chemical speciation results for a series of common aromatic and halogenated mixtures eluting from a GC using a millisecond pulsed GD with time-gated acquisition. In preliminary experiments, helium was employed as the GC carrier gas and an argon auxiliary gas was introduced into the GD chamber. This resulted in a GD plasma consisting of 50\% helium and 
$50 \%$ argon and, unfortunately, irreproducible molecular data. Alternatively, helium has been considered for use as the GC carrier gas along with the creation of an all helium plasma. However, helium has much higher metastable energies than argon, thereby increasing the degree of fragmentation and decreasing the ability to obtain the analyte's molecular ion. Taking this into consideration, argon was successfully used as both the GC carrier gas and GD plasma gas in experiments performed by Lewis et al.

Further investigations using GD-MS as a detector for compounds eluting from a GC have been shown to provide comprehensive chemical analysis. ${ }^{2730}$ Despite this attention to GC/GD-MS in the chemical speciation of compounds, the ability of GC/GD-MS to solve the practical problems presented by chemists has lagged far behind and to date there is only one publication ${ }^{31}$ to the author's knowledge. Because the technique has shown such promise, the research goal of this chapter is to expand its application to the analysis of CWA metabolites.

\subsection{Materials and methods}

\subsubsection{Materials}

The nerve agent breakdown products, IMPA, PMPA, and EMPA, were purchased dissolved in methanol from Cerilliant (Round Rock, TX). These compounds had a concentration of $1000 \mathrm{\mu g} / \mathrm{mL}$ in methanol. Although IMPA, PMPA, and EMPA were available, IMPA was chosen as the test analyte based on the significance of its corresponding nerve agent, sarin. The derivatizing agent N-methyl-N-(tertbutyldimethylsilyl)-trifluoroacetamide ( $>97 \%$ purity) was acquired from Sigma-Aldrich (St. Louis, MO). Both nerve agent metabolites and derivatizing agent were stored in a freezer and were allowed to warm to room temperature before use. Due to the fact that AMPA readily degrades to MPA, all solutions were made just prior to their analysis. The sample matrix consisted of synthetic urine that was purchased from Alltech (Deerfield, IL). The $100 \mu \mathrm{m}$ PDMS, $75 \mu \mathrm{m}$ Carboxen-PDMS, and $65 \mu \mathrm{m}$ PDMS/DVB solid phase microextraction fibers and all fiber injection equipment and accessories were obtained from Sigma-Aldrich (St. Louis, MO). Ultrapure, deionized (18.2 M $\Omega$ ) water was obtained from a Milli-Q water purification system, Model Gradient A-10 (Millipore, Bedford, MA). 


\subsubsection{Sample preparation}

The phosphonic acid degradation products of nerve agents contain functional groups (e.g. alcohol moiety) making them highly polar and of low volatility. ${ }^{[32}$ In order to be amenable to GC analysis, these molecules must be derivatized into volatile, nonpolar compounds. ${ }^{33}$ There exists a number of derivatization methods applicable to the hydrolysis products of CWAs: silylation by N,O-bis-trimethylsilyl-trifluoroacetamide (BSTFA) and N-methyl-N-(tert-butyldimethylsilyl)-trifluoroacetamide (MTBSTFA), diazoalkylation by diazomethane, 21 and pentafluorobenzylation by pentafluorobenzyl bromide. However, diazomethane is a toxic and potentially explosive compound and the derivatization reaction using pentafluorobenzyl bromide requires several hours. 34

Silylation is the most widely used derivatization technique for gas chromatographic applications and is useful for a wide variety of compounds. $\frac{35 \mid 36}{36}$ Formerly, BSTFA was the preferred derivatization reagent for silylation, which produced a trimethylsilyl (TMS) derivative. However, anhydrous conditions (the presence of moisture results in poor reaction yield and instability of the derivatized analytes 37 ) and continuous heating were required $\stackrel{38}{~ R e a g e n t s, ~ s u c h ~ a s ~ M T B S T F A, ~ t h a t ~ i n t r o-~}$ duce a tert-butyldimethylsilyl (TBDMS) group instead of the TMS group were developed. ${ }^{39}$ MTBSTFA derivatives are less moisture sensitive and more stable than those formed using lower molecular weight reagents such as BSTFA. $.36 / 38 / 40$ Additionally, it has been found that for target compounds without sterically hindered sites, such as IMPA, MTBSTFA is preferred due to generally higher analytical responses. $35[41$

In this study, derivatization was accomplished using MTBSTFA as the derivatizing agent. MTBSTFA is categorized as a silylation derivatization in which, in the case of AMPAs, acidic hydrogens in - $\mathrm{OH}$ are replaced with a TBDMS group producing a silyl derivative which, compared to its parent compound, is more volatile, less polar, and more thermally stable. Figure 3.2 shows the derivatization reaction of IMPA with MTBSTFA to form a TBDMS derivative when reacted with polar functional groups containing an acidic hydrogen. Replacement of an acidic hydrogen with a TBDMS group adds 114 to the molecular weight.

\subsubsection{Instrumentation}

Analysis was carried out on a Varian 3400 GC equipped with a split/splitless injector and a HP-5MS, $30 \mathrm{~m}$ x $0.25 \mathrm{~mm}$ i.d., fused silica capillary column (Agilent, Santa Clara, CA). The carrier gas, argon, had a linear velocity of $35 \mathrm{~cm} /$ second. The linear velocity within the GC was determined using butane gas from a disposable 


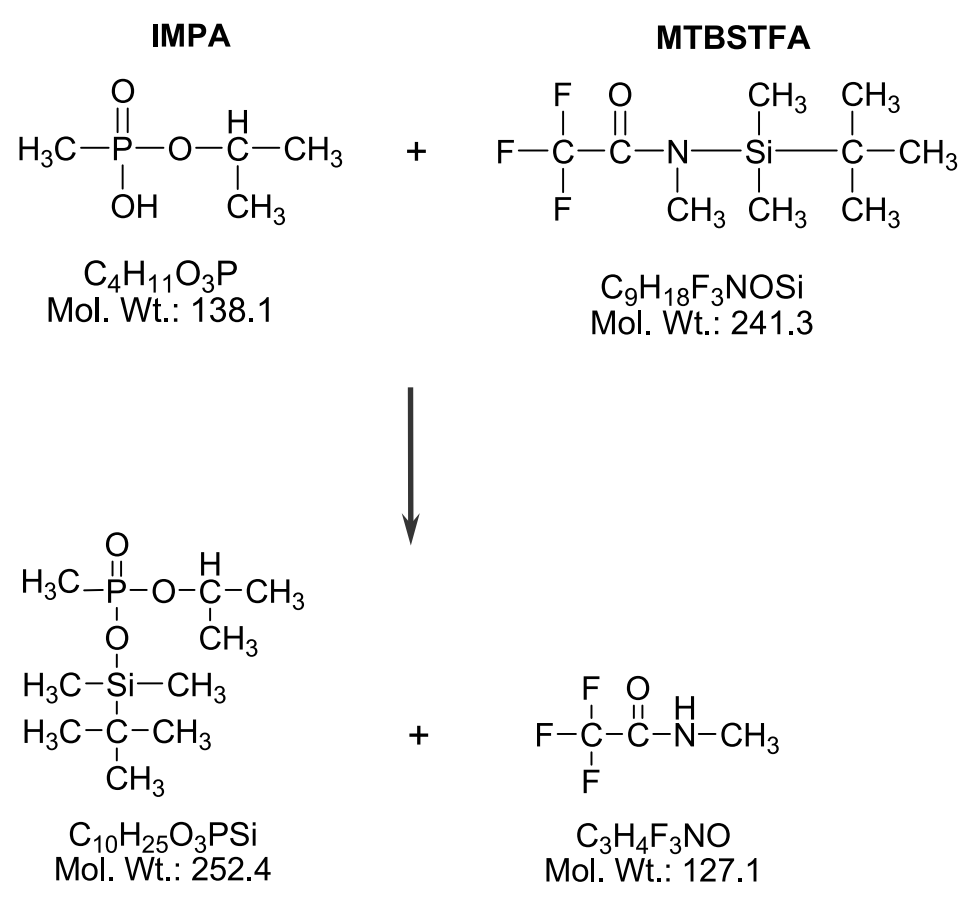

Figure 3.2: Derivatization of IMPA with MTBSTFA. 
lighter. Using a gas-tight syringe (Hamilton, Reno, NV), a vapor injection of $1-2 \mu \mathrm{L}$ of the unretained compound was made and a retention time obtained. The carrier gas linear velocity was controlled by adjusting the head pressure. The split/splitless injection port was held at $250{ }^{\circ} \mathrm{C}$. The oven was held at $100{ }^{\circ} \mathrm{C}$ for $1 \mathrm{~min}$. At 1 min the oven temperature was ramped at a rate of $30{ }^{\circ} \mathrm{C} / \mathrm{min}$ to a final temperature of $280{ }^{\circ} \mathrm{C}$, resulting in a run time of 22 minutes. To ensure compatibility with the GD, ultrapure argon (Airgas, Randor, PA) was used as both the carrier gas for the GC and the discharge support gas for the GD plasma. $\frac{26}{2}$ The original detector of the $\mathrm{GC}$ had been removed. Coupling of the GC to the GD chamber was achieved by adapting the GC oven to accommodate a temperature-controlled copper transfer line through which the GC capillary column was fed. The copper transfer line from the GC to the GD chamber was wrapped in heating tape with an adjustable thermostat to heat the transfer line to a constant $150{ }^{\circ} \mathrm{C}$. The GC capillary then exited the transfer line and was introduced into the GD chamber via a specially made fitting consisting of a Swagelok welded onto the outside of a double-sided flange through which a stainless steel tube (1 mm i.d.) was inserted to act as the GC capillary guide. The steel tube was extended into the GD sample chamber parallel to the GD probe. The GC capillary column that protruded out from the steel tube was angled toward the cathode's surface with the aid of a wire that was coiled and bent. The capillary end was positioned approximately $2 \mathrm{~mm}$ from the ion exit orifice. An illustration of the introduction of GC effluent to the GD plasma is shown in Figure 3.3. The temperature programming conditions for the GC and typical operating conditions for the GD and TOF are listed in Table 3.1.

The GC was coupled to a laboratory-constructed GD ion source with a linear time-of-flight (TOF) mass analyzer (R.M. Jordan Co., Grass Valley CA) oriented orthogonally to the GD ion beam. A six-way, high vacuum cross (MDC Vacuum Products Co., Hayward, CA) served as the GD chamber and plasmas were generated using a direct insertion probe (DIP) onto which a silver disk cathode $(5 \mathrm{~mm}$ diameter, $2 \mathrm{~mm}$ thickness) was mounted. The DIP allows for axial movement inside the GD chamber enabling different distances of the cathode sampling surface from the TOF sampling orifice to be measured. Further information on the design of this instrumentation can be found in previous papers published by this group. $\frac{42 / 43}{4}$

The plasma was formed by ultrapure argon with discharge gas chamber pressures ranging $0.4-1.2$ Torr. A $13.56 \mathrm{MHz}$ radio frequency (rf) generator coupled with an automatic matching network (RF Plasma Product Inc., Marlton, NJ) was used to power the GD plasma. The GD was operated in rf pulsed mode using a pulse duration of $5 \mathrm{~ms}$ with a $25 \%$ duty cycle resulting in a pulse cycle of $20 \mathrm{~ms}$. The 
Table 3.1: GC/Pulsed GD-TOFMS Operating Conditions

\begin{tabular}{ll}
\hline Gas Chromatograph & \\
\hline Stationary phase & HP-5MS \\
Column dimensions & $30 \mathrm{~m} \mathrm{x} 0.25 \mathrm{~mm}$ i.d. \\
Film thickness & $0.25 \mu \mathrm{m}$ \\
Carrier gas & argon \\
Injector temp & $250^{\circ} \mathrm{C}(1 \mathrm{~min})$ \\
Initial temperature & $100{ }^{\circ} \mathrm{C}$ \\
Ramp rate & $30{ }^{\circ} \mathrm{C} / \mathrm{min}$ \\
Final temperature & $280{ }^{\circ} \mathrm{C}(1 \mathrm{~min})$ \\
\hline Pulsed Glow Discharge & \\
\hline Cathode material & silver \\
Pressure: discharge gas (Ar) & $0.4-1.2 \mathrm{Torr}$ \\
Pulse power & $40-100 \mathrm{~W}$ \\
Pulse width & $5.0 \mathrm{~ms}$ \\
Pulse duty cycle & $25 \%$ \\
Distance from cathode to ion exit orifice & $2-12 \mathrm{~mm}$ \\
\hline Time-of-Flight Mass Analyzer & \\
\hline 1st vacuum stage pressure & $10^{-3} \mathrm{Torr}$ \\
2nd vacuum stage pressure & $10^{-6} \mathrm{Torr}$ \\
Repeller pulse width & $1 \mu \mathrm{s}$ \\
Skimmer potential & $-1225 \mathrm{~V}$ \\
Slit potential & ground \\
Repeller potential & $+225 \mathrm{~V}$ \\
Repeller bias potential & -8 to $-15 \mathrm{~V}$ \\
Extractor grid potential & ground \\
Accelerator / flight tube potential & $-1550 \mathrm{~V}$ \\
X steering plate potential & $-1880 \mathrm{~V}$ \\
Y steering plate potential & $-1560 \mathrm{~V}$ \\
Micro-channel plate detector potential & $-1830 \mathrm{~V}$ \\
\hline
\end{tabular}




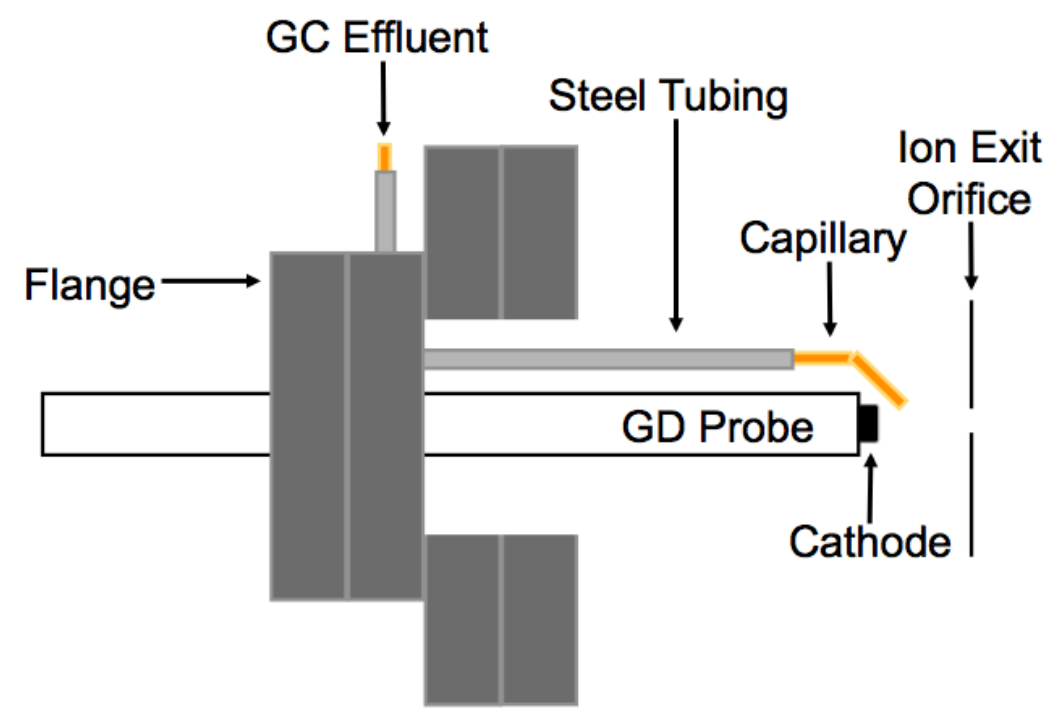

Figure 3.3: Schematic diagram of the GC-GD interface.

operating rf power varied between 40-100 W, limited by the threshold value above which the discharge becomes unstable and forms an arc.

A digital delay generator (DDG) (EG\&G Princeton Applied Research, Princeton, NJ) in synchronization with the GD rf power pulse was used to trigger a $1 \mu \mathrm{s}$ ion extraction pulse to the TOF repeller thereby initiating the signal detection system. The DDG allows for time-gated detection with which mass spectra can be obtained at selected temporal intervals within the power pulse sequence. Mass spectra were acquired during the plateau at $4.8 \mathrm{~ms}$ after pulse ignition and during the afterpeak at $0.2 \mathrm{~ms}$ after the pulse termination.

The mass spectrometric data was amplified and fed into a 1-GHz oscilloscope (Lecroy 9370M, Chestnut Ridge, NY) for collection. The oscilloscope averaged the spectra continuously with 1:63 weighting resulting in an averaging window of 1.26 s. The mass spectra were transferred from oscilloscope to the computer through a GPIB card and saved in ASCII format. A Microsoft Excel (97 spreadsheet) macro tool developed by the group was used to open the ASCII data, convert time to $\mathrm{m} / \mathrm{z}$, calibrate the $m / z$, and plot the mass spectrum. 


\subsection{Results and discussion}

\subsubsection{Liquid injections}

Preliminary work with liquid injections was performed to reduce the complexity of the method and to assess performance of the GC and pulsed GD-TOFMS. In these preliminary experiments, equal volumes of IMPA and MTBSTFA were combined in a glass vial and the sample was mixed using a vortex mixer for $30 \mathrm{~s}$ at a moderate speed. After the mixture stood at room temperature for $15 \mathrm{~min}, 1-2 \mu \mathrm{L}$ volumes were manually injected using a $5 \mu \mathrm{L}$ gas-tight syringe into the GC injection port using a split ratio of 50:1. Split injection mode was used to remove the bulk of the methanol solvent and thereby reduce plasma quenching.

Figure 3.4 shows blank and analyte mass spectra obtained from GC/GD-TOFMS with time-gated detection. Both GD plateau data and afterpeak data were investigated, but only the plateau regime showed promise. The characteristic fragment ion for derivatized IMPA, $\mathrm{C}_{6} \mathrm{H}_{15} \mathrm{OSi}^{+}$at $m / z 131$ was detected during the plateau of the pulsed GD. In comparison, a study by Moser et al. ${ }^{44}$ using GC/MS with electron impact, found $\mathrm{C}_{5} \mathrm{H}_{14} \mathrm{O}_{3} \mathrm{P}^{+}$at $m / z 153$ and $\mathrm{C}_{6} \mathrm{H}_{16} \mathrm{O}_{3} \mathrm{PSi}^{+}$at $m / z 195$ to be characteristic of derivatized IMPA.

The ion signal at $m / z 131$ was chosen to assess the change in intensity as a function of discharge gas pressure. The discharge pressures were varied from 600 to $2000 \mathrm{mTorr}$, while the rf power and cathode-anode spacing remained constant. The effect of discharge gas pressure is shown in Figure 3.5. The plasma of the GD undergoes a complex series of changes as the pressure is increased. Not only does pressure influence the spatial regions of the GD plasma, but also changes the fundamental plasma properties. It is well known that Penning ionization peaks at intermediate pressures, whereas electron ionization is favored at lower pressures. The decrease in the ion at $m / z 131$ indicates a reduced metastable population and a decrease in Penning ionization with increased pressure. As a result, the ionization efficiency drops.

\subsubsection{Solid phase microextraction}

The detection of urinary metabolites may provide the simplest means of confirming an exposure to a nerve agent. Analysis of urine has the advantage of being a less complex matrix and a less intrusive sample than blood or hair, but still requires ex- 


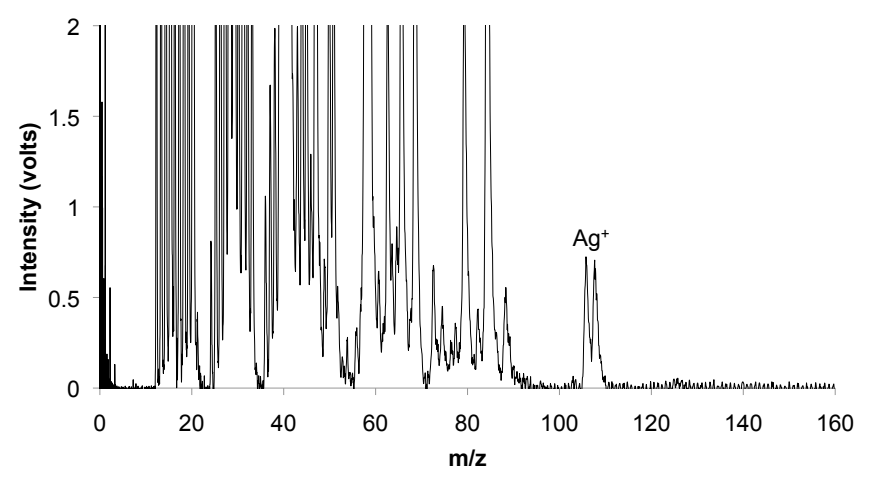

(a) Blank methanol and MTBSTFA sample. r.t. $5.2 \mathrm{~min}$

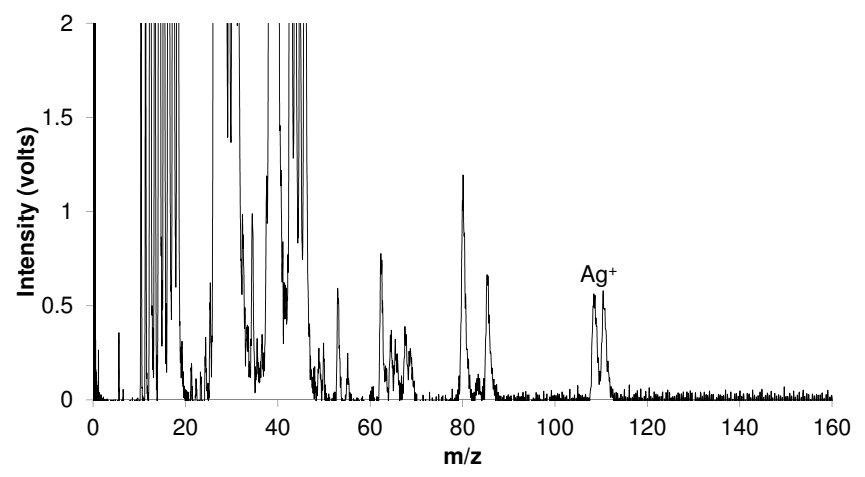

(b) IMPA and MTBSTFA sample. r.t. $4.8 \mathrm{~min}$

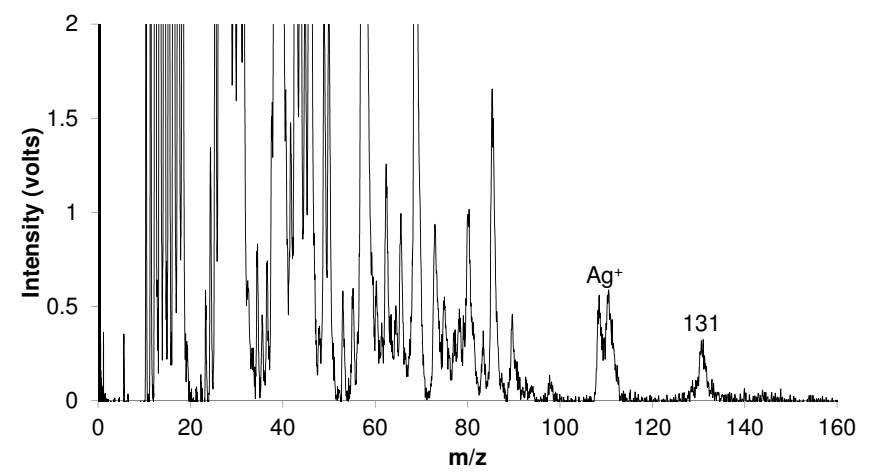

(c) IMPA and MTBSTFA sample. r.t. 5.0 min

Figure 3.4 


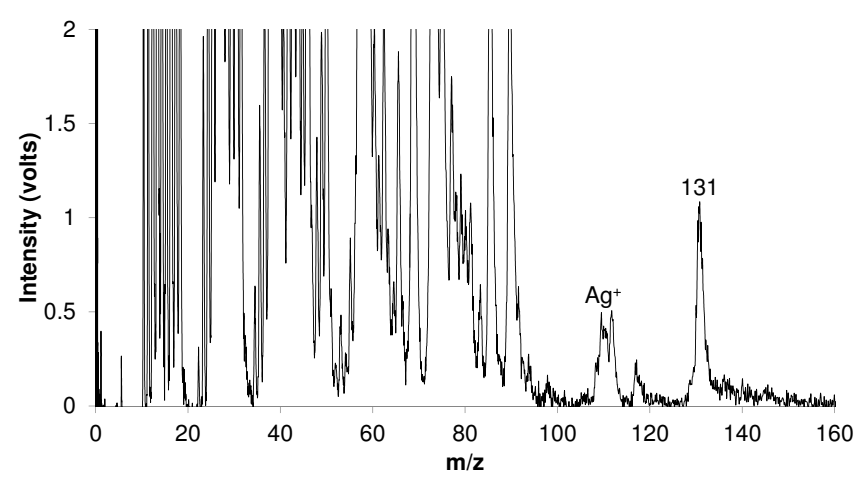

(d) IMPA and MTBSTFA sample. r.t. $5.2 \mathrm{~min}$

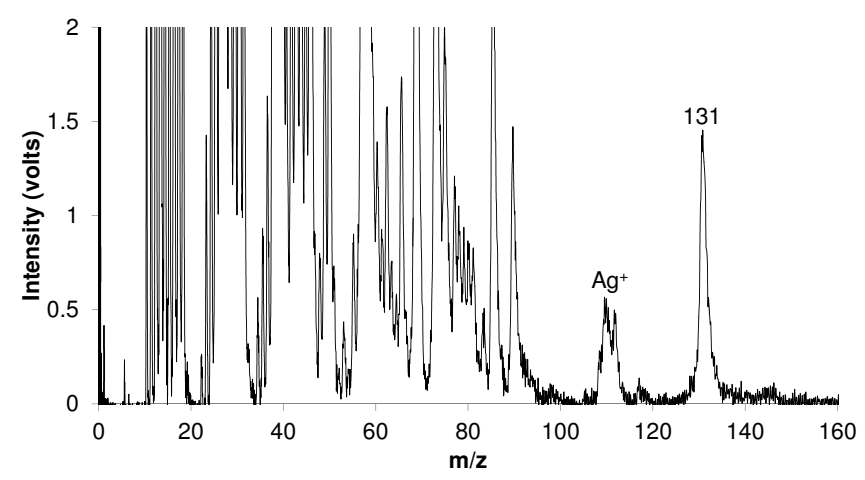

(e) IMPA and MTBSTFA sample. r.t. $5.4 \mathrm{~min}$

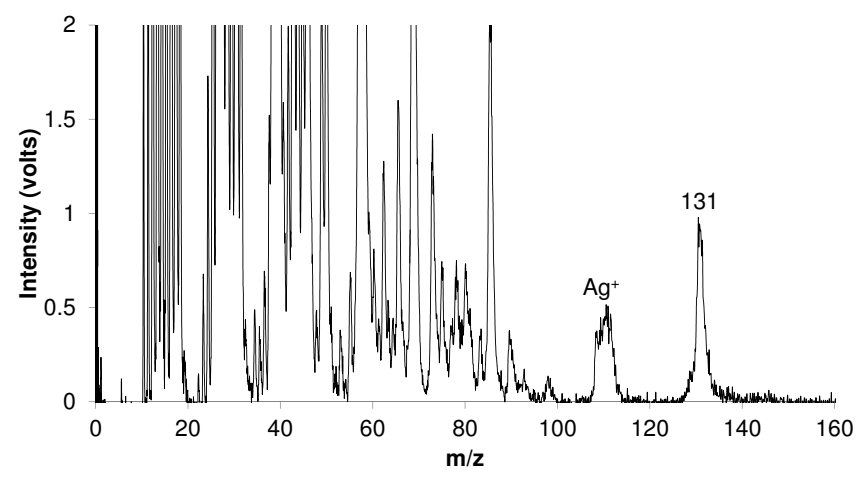

(f) IMPA and MTBSTFA sample. r.t. $5.6 \mathrm{~min}$

Figure 3.4 


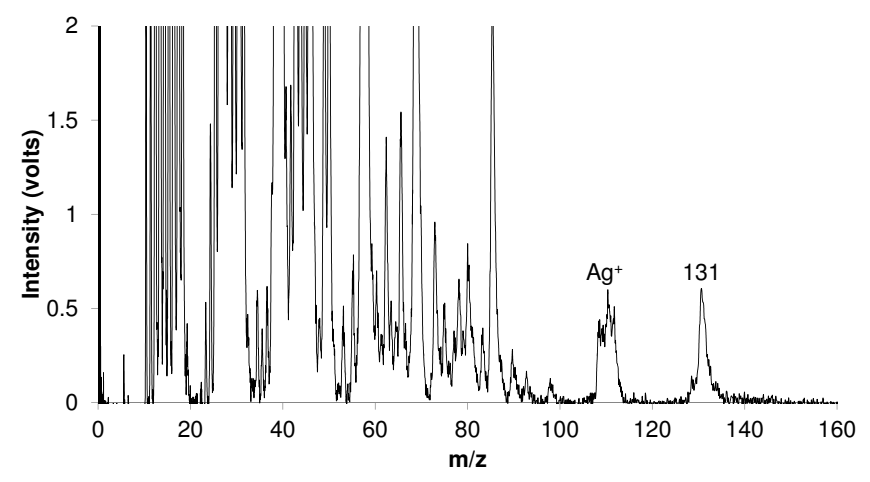

(g) IMPA and MTBSTFA sample. r.t. $5.8 \mathrm{~min}$

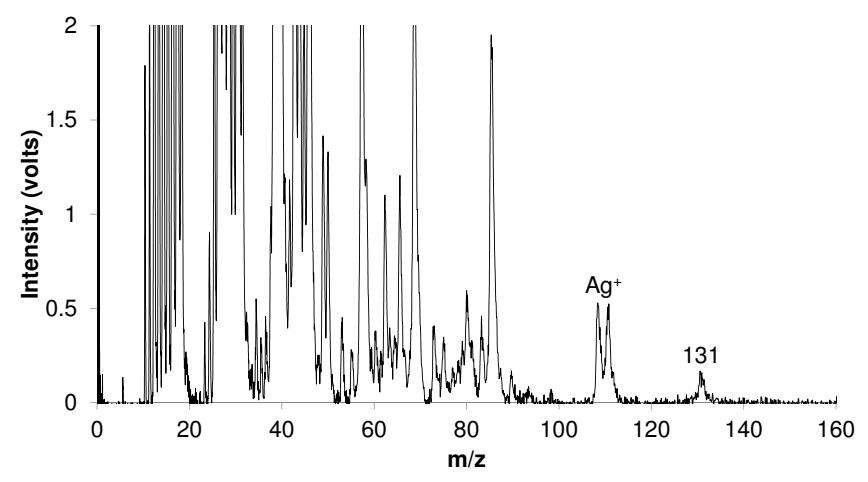

(h) IMPA and MTBSTFA sample. r.t. 6.0 min

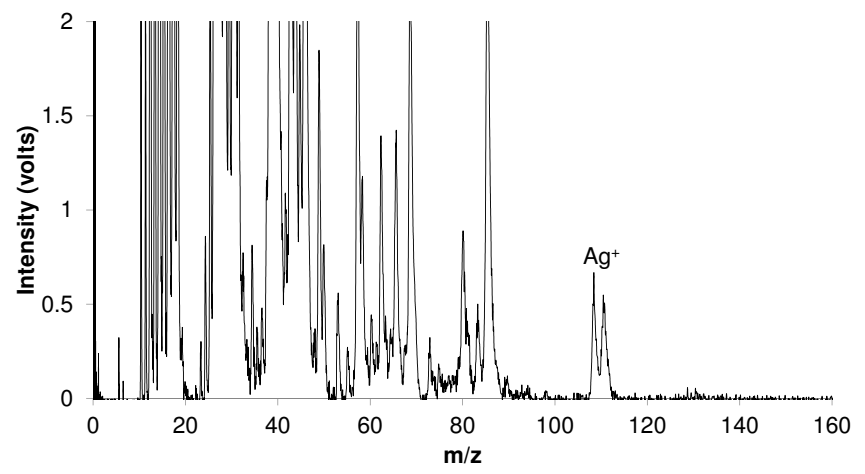

(i) IMPA and MTBSTFA sample. r.t. $6.2 \mathrm{~min}$

Figure 3.4: Mass spectrum of methanol and MTBSTFA sample blank and mass spectra of derivatized IMPA sample. $500 \mu \mathrm{g} / \mathrm{mL}$ IMPA, liquid injection $1 \mu \mathrm{L}$, silver cathode, plateau, 600 mTorr, $80 \mathrm{~W}$ rf power. 


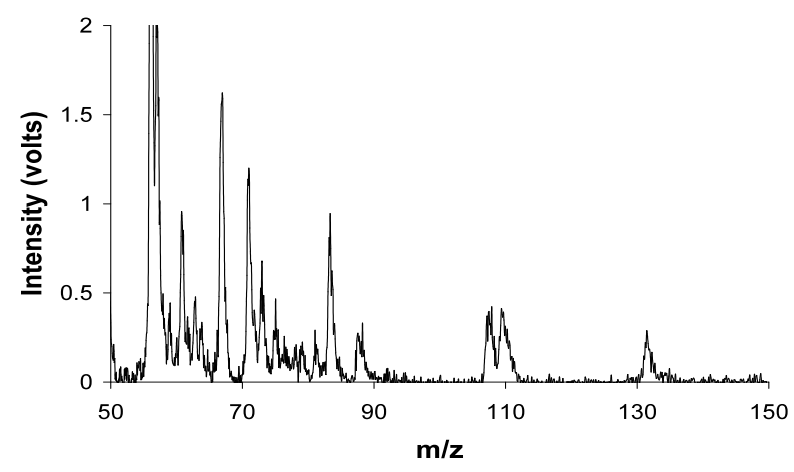

(a) 600 mTorr

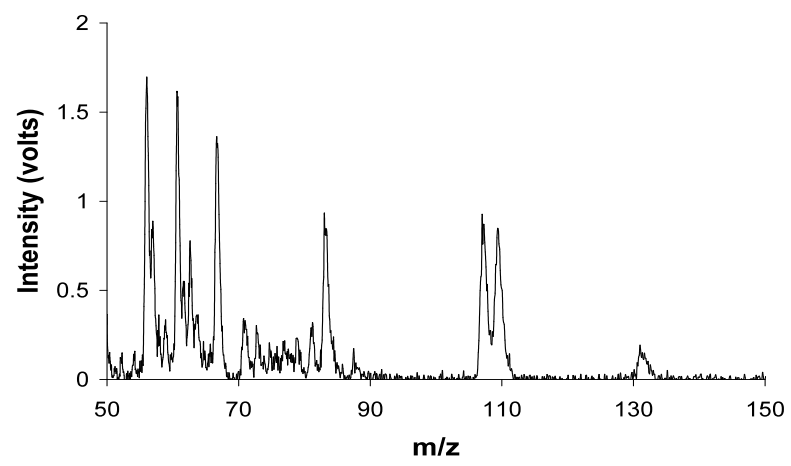

(b) 800 mTorr

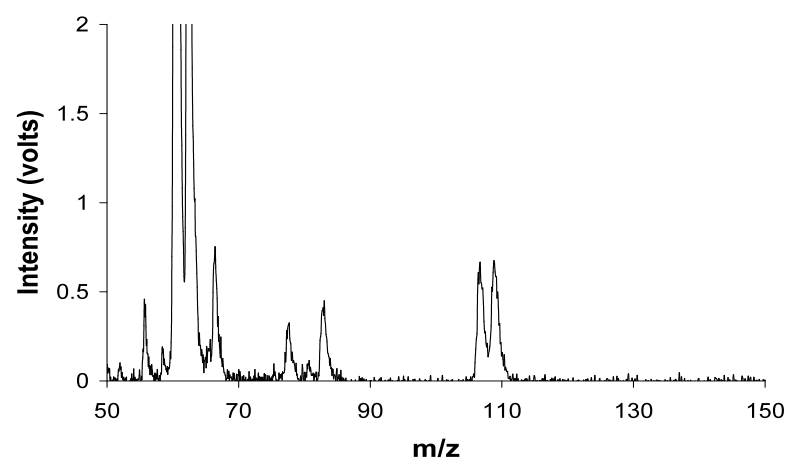

(c) 2000 mTorr

Figure 3.5: Effect of discharge gas pressure on mass spectra of derivatized IMPA. Liquid injection $1 \mu \mathrm{L}$, r.t. $5.2 \mathrm{~min}$, silver cathode, plateau, $80 \mathrm{~W}$ rf power. 
traction procedures to minimize matrix effects and obtain high recoveries. Analysis of urine also has the advantage that urine biomarkers are generally not regulated, do not require special permits or laboratory facilities to use, can be purchased commercially or synthesized, and the isotopically labeled compounds (for use as internal standards) are readily available. Urine has the disadvantages of containing potential non-unique biomarkers for some CWA and the loss of biomarkers within a few days of initial exposure.

Solid phase microextraction (SPME) is a simple and solventless extraction procedure. The fiber can be directly inserted into a GC injection port where thermal desorption of the collected analytes occurs, permitting the efficient transfer of the compounds for GC/MS analysis. SPME has proved to be effective in extracting AMPAs from aqueous matrices. $\frac{17}{}$ For ionization by GD, SPME samples are preferred over their liquid counterparts as GDs require low solvent loading to avoid quenching of the plasma.

Before use, each SPME fiber was conditioned for $1 \mathrm{hr}$ at $250{ }^{\circ} \mathrm{C}$ in the injection port of the GC. Samples of synthetic urine, $3 \mathrm{~mL}$, were contained in a 4 -mL glass vial with a screw top and Teflon-faced silicone septum. IMPA was spiked into the synthetic urine sample, resulting in analyte concentrations in the $\mu \mathrm{g} / \mathrm{mL}$ range. To the sample solution, $25 \%$ (wt./vol.) sodium chloride was added to reduce the analyte solubility and conc. hydrochloric acid was added to keep the solution at a $\mathrm{pH}$ of 2 . To aid in mass transport and reduce equilibrium times, Teflon-coated magnetic stir bars were inserted into the vial and the vial placed on a magnetic stirrer. The SPME fiber was immersed into the sample solution to extract analytes with times ranging from 15-30 min then withdrawn. The same fiber was then exposed to the headspace of an enclosed vial containing $3 \mathrm{~mL}$ MTBSTFA with times ranging from 15-30 min, also with constant stirring. The fiber was inserted into the splitless injection port of the GC for $1 \mathrm{~min}$ before the split vent valve was opened.

All three SPME fiber coatings were tested. A minimum of three extractions were performed with each coating to determine which gave the best response. It was expected that the Carboxen-PDMS and PDMS/DVB fibers would demonstrate improved extraction efficiencies over the PDMS fiber, however, minimal differences were noted. Variations in the duration of extraction and derivatization also did not demonstrate any improvements. Mass spectra from SPME sampling resulted in mass spectra very similar to that of the liquid injections; a peak at $m / z 131$. These findings indicate that the GC/GD-TOFMS instrument requires improvement before optimization of the sample preparation process can be explored. 


\subsection{Conclusions and future directions}

Assessing the use of CWAs in war or acts of terrorism requires validated methods for the accurate and rapid analysis of biological samples to identify and determine level of exposure to these deadly agents. The ultimate goal of this study was to expand the utility of the pulsed glow discharge through the development of a confirmative technique for the analysis of organophosphorous nerve agent metabolites in urine to monitor CWA exposure. This study attempted to combine SPME, GC, and a pulsed GD-TOFMS in the development of a new method for the analysis of CWA metabolites in urine samples. The sample prep and GC conditions were directed by optimized techniques found in the literature for the phosphonic acids. The optimization of glow discharge conditions was explored for the detection of the analytes of interest by testing different gas discharge pressures and GD time regimes. This study demonstrates that with the proper use of derivatization reagents such as MTBSTFA, phosphonic acids can be analyzed by GC/GD-MS. The reaction conditions may have to be "tweaked" to produce maximum response of the derivatives of interest. The derivatives produced characteristic fragments, allowing for easy identification by MS. However, the mass spectra obtained for eluting compounds did not yield both structural and molecular information as was anticipated. Future directions would focus on improving the analysis method through a variety of troubleshooting techniques.

For mass spectrometry, water, hydrocarbons, oxygen, and other contaminants from the gas supply will lead to column degradation, background noise, and unwanted ion-molecule reactions. This can greatly complicate the spectra, making interpretation, even of molecular ion identity, very difficult. Moisture traps, hydrocarbon traps, and oxygen traps can be used in-line to prevent such an occurrence. Traps should always be used, even with ultra high purity gases, to prevent impurities from entering the GC system. Although traps were in-line on the GC, their functionality was unknown. The method could potentially have benefited from the purchase and installation of new gas traps.

The location of optimum gas velocity is different for various gases (nitrogen at $\sim 12 \mathrm{~cm} / \mathrm{sec}$, helium at $\sim 20 \mathrm{~cm} / \mathrm{sec}$, hydrogen at $\sim 40 \mathrm{~cm} / \mathrm{sec}$ ). From the different van Deemter curves for nitrogen, helium, and hydrogen, it is evident that 'heavy' carrier gases in the optimum generate slightly higher efficiencies. However, this optimum is obtained at a low gas velocity and the analysis time is longer. Increasing the gas velocity for nitrogen, in view of the slope of the curve, will result in a considerable loss of plates. Like nitrogen, argon is considered a 'heavy' carrier gas and as such its linear velocity is best adjusted to values near that of nitrogen. In this study, argon, 
the carrier gas, was set at a linear velocity of $35 \mathrm{~cm} /$ second; attempts to decrease the head pressure any further resulted in zero head pressure. Column efficiency could be improved by adjusting the linear velocity to a value closer to that of nitrogen.

The Varian 3400 GC is limited to constant pressure mode. Operating in the constant pressure mode and the use of a temperature program often requires efficiency compromises since the average linear velocity decreases when the temperature of the column increases. For this reason, it is common practice to set the linear velocity above that of optimal. Regardless of the average linear velocity used, it will not be optimal for all compounds within a mixture. An improvement would be to use a GC with a pressure programmable injector to automatically increase the carrier gas pressure, thus maintaining a constant linear velocity throughout a temperature gradient run. An added advantage would be a decrease in retention times for any later eluting compounds.

The capillary column, from GC oven exit to GD plasma introduction, should be temperature-controlled as much as possible to prevent any analyte condensation in the transfer line or GD cell interior. The coupling of GC to GD by Olsen et al. $\stackrel{24}{ }$ highlighted the importance of the GC/GD interface design. In their initial interface design, inadequate heating of the GC capillary column resulted in only one broad chromatographic peak, yet a mixture of two compounds were injected. This method could benefit by moving the interface from GC to GD chamber closer to that of the plasma, thereby leaving the bare minimum length of capillary exposed and minimizing analyte condensation and peak broadening.

It has been shown that tuning the discharge to maximum sensitivity of species such as the sputtered cathode material does not necessarily reflect the maximum sensitivity for transient organic analytes. $\frac{30}{30}$ This method could benefit from optimization of the GD source parameters for the specific analytes. Such optimization studies have been done in the past. ${ }^{27}$ But, because ionization efficiency depends on factors such as the nature of the cathode and the discharge gas, pressure, cell geometry, and distance between electrodes, it is difficult to directly compare one source with another and each design will have its own characteristics. Therefore, prior studies should only be used as guidelines and optimization performed on a case-by-case basis.

The GD chamber used in this study was comprised of all solid stainless steel. Because the position of the analyte molecules and ionization mechanisms with respect to the exit orifice is of critical importance, the method would benefit from a quartz window/viewport allowing for the visual adjustment of the capillary column within the GD chamber. The stainless-steel tube should be positioned at an angle 
which would align the capillary column outlet with the sampling orifice of the mass spectrometer.

The molecular ion of derivatized IMPA is $\mathrm{C}_{10} \mathrm{H}_{25} \mathrm{O}_{3} \mathrm{PSi}^{+}$at $m / z 252$. However, this particular TOF suffers from poor resolving power at higher masses. Improvements could be made to the TOF's acceleration plates or the incorporation of a reflectron could greatly improve resolution.

Once the GC is successfully coupled with the plasma source, the experiment becomes a simple time-resolved analysis. That is, the spectrometer output for a given analyte must be plotted as a function of elution time, in real time. Because gas chromatographic peaks are typically less than 10 seconds in duration, the rapid acquisition of mass spectrometric data is crucial. Perhaps the most effective way of accomplishing this would be to create a software program (e.g. LabVIEW) to automatically collect retention time and corresponding mass spectra over the course of the elution. Unfortunately, a previous LabVIEW program, developed and implemented by this group, was insufficient in its ability to accurately record data and was taxing on the computer, filling its hard drive after $12 \mathrm{~min}$ of data acquisition. Future success would require software to collect and process the data in combination with a larger hard drive and/or smaller/fewer data files.

In order to reduce analysis times and take advantage of the full ability of the pulsed GD, additional DDGs and oscilloscopes could be integrated into the instrumental design. This would allow for the concurrent collection of elemental and molecular data collected from different time regimes all within a single GC/GD-TOFMS analysis. 


\section{Bibliography}

[1] <http://www.bt.cdc.gov/agent/sarin/basics/facts.asp> accessed 5 July 2009.

[2] "Assay techniques for detection of exposure to sulfur mustard, cholinesterase inhibitors, sarin, soman, GF, and cyanide." Technical Bulletin, 1996.

[3] E. R. J. Wils and A. G. Hulst, "Determination of organophosphorous acids by thermospray liquid chromatography-mass spectrometry," Journal of Chromatography A, vol. 454, pp. 261-272, 1988.

[4] R. M. Black and R. W. Read, "Analysis of degradation products of organophosphorus chemical warfare agents and related compounds by liquid chromatography-mass spectrometry using electrospray and atmospheric pressure chemical ionisation," Journal of Chromatography A, vol. 794, pp. 233-244, 1998.

[5] P. A. D'Agostino, J. R. Hancock, and L. R. Provost, "Packed capillary liquid chromatography-electrospray mass spectrometry analysis of organophosphorus chemical warfare agents," Journal of Chromatography A, vol. 840, no. 2, pp. 289294, 1999.

[6] R. W. Read and R. M. Black, "Rapid screening procedures for the hydrolysis products of chemical warfare agents using positive and negative ion liquid chromatography-mass spectrometry with atmospheric pressure chemical ionisation," Journal of Chromatography A, vol. 861, no. 2, pp. 169-177, 1999.

[7] P. A. D'Agostino, J. R. Hancock, and L. R. Provost, "Determination of sarin, soman and their hydrolysis products in soil by packed capillary liquid chromatography-electrospray mass spectrometry," Journal of Chromatography A, vol. 912, no. 2, pp. 291-299, 2001.

[8] J. R. Smith and M. L. Shih, "Analysis of the degradation compounds of chemical warfare agents using liquid chromatography/mass spectrometry," Journal of Applied Toxicology, vol. 21, pp. S27-S34, 2001.

[9] P. A. D'Agostino, C. L. Chenier, and J. R. Hancock, "Packed capillary liquid chromatography-electrospray mass spectrometry of snow contaminated with sarin," Journal of Chromatography A, vol. 950, pp. 149-156, 2002. 
[10] K. M. Kubachka, D. D. Richardson, D. T. Heitkemper, and J. A. Caruso, "Detection of chemical warfare agent degradation products in foods using liquid chromatography coupled to inductively coupled plasma mass spectrometry and electrospray ionization mass spectrometry," Journal of Chromatography A, vol. 1202, no. 2, pp. 124-131, 2008.

[11] R. M. Black and R. W. Read, "Application of liquid chromatographyatmospheric pressure chemical ionisation mass spectrometry, and tandem mass spectrometry, to the analysis and identification of degradation products of chemical warfare agents," Journal of Chromatography A, vol. 759, pp. 79-92, 1997.

[12] T. L. Hayes, D. V. Kenny, and L. Hernon-Kenny, "Feasibility of direct analysis of saliva and urine for phosphonic acids and thiodiglycol-related species associated with exposure to chemical warfare agents using LC-MS/MS," Journal of Medical Chemical Defense, vol. 2, pp. 1-23, 2004.

[13] L. L. Swaim, R. C. Johnson, Y. Zhou, C. Sandlin, and J. R. Barr, "Quantification of organophosphorus nerve agent metabolites using a reduced-volume, high-throughput sample processing format and liquid chromatography-tandem mass spectrometry," Journal of Analytical Toxicology, vol. 32, pp. 774-777, 2008.

[14] D. J. Harvey and M. G. Horning, "Derivatives for the characterization of alkyland aminoalkylphosphonates by gas chromatography and gas chromatographymass spectrometry," Journal of Chromatography A, vol. 79, pp. 65-74, 1973.

[15] J. G. Purdon, J. G. Pagotto, and R. K. Miller, "Preparation, stability and quantitative analysis by gas chromatography and gas chromatography-electron impact mass spectrometry of tert-butyldimethylsilyl derivatives of some alkylphosphonic acid and alkyl methylphosphonic acids," Journal of Chromatography A, vol. 475, pp. 261-272, 1989.

[16] M. L. Shih, J. R. Smith, J. D. McMonagle, T. W. Dolzine, and V. C. Gresham, "Detection of metabolites of toxic alkylmethylphosphonates in biological samples," Biological Mass Spectrometry, vol. 20, no. 11, pp. 717-723, 1991.

[17] M. T. Sng and W. F. Ng, "In-situ derivatisation of degradation products of chemical warfare agents in water by solid-phase microextraction and gas chromatographic-mass spectrometric analysis," Journal of Chromatography A, vol. 832 , pp. $173-182,1999$. 
[18] D. D. Richardson and J. A. Caruso, "Derivatization of organophosphorous nerve agent degradation products for gas chromatography with ICPMS and TOF-MS detection," Analytical and Bioanalytical Chemistry, vol. 388, no. 4, pp. 809-823, 2007.

[19] O. Terzic, "Screening of degradation products, impurities and precursors of chemical warfare agents in water and wet or dry organic liquid samples by in-sorbent tube silylation followed by thermal desorption-gas chromatographymass spectrometry," Journal of Chromatography A, vol. 1217, no. 30, pp. 49874995, 2010.

[20] P. A. D'Agostino and C. J. Porter, "Capillary column gas chromatography/tandem mass spectrometry verification of chemical warfare agents," Rapid Communications in Mass Spectrometry, vol. 6, no. 11, pp. 717-718, 1992.

[21] J. R. Barr, W. J. Driskell, L. S. Aston, and R. A. Martinez, "Quantitation of metabolites of the nerve agents sarin, soman, cyclohexylsarin, VX, and Russian VX in human urine using isotope-dilution gas chromatography-tandem mass spectrometry," Journal of Analytical Toxicology, vol. 28, pp. 372-378, 2004.

[22] R. E. Steiner, C. L. Lewis, and V. Majidi, "Consideration of a millisecond pulsed glow discharge time-of-flight mass spectrometer for concurrent elemental and molecular analysis," Journal of Analytical Atomic Spectrometry, vol. 14, pp. 1537-1541, 1999.

[23] V. Majidi, M. A. Moser, C. L. Lewis, W. Hang, and F. L. King, "Explicit chemical speciation by microsecond pulsed glow discharge time-of-flight mass spectrometry: concurrent acquisition of structural, molecular and elemental information," Journal of Analytical Atomic Spectrometry, vol. 15, no. 1, pp. 19-25, 2000 .

[24] L. K. Olson, M. Belkin, and J. A. Caruso, "Radiofrequency glow discharge mass spectrometry for gas chromatographic detection: a new departure for elemental speciation studies," Journal of Analytical Atomic Spectrometry, vol. 11, pp. 491496, 1996.

[25] M. A. Belkin, L. K. Olson, and J. A. Caruso, "Radiofrequency glow discharge as an ion source for gas chromatography with mass spectrometric detection," Journal of Analytical Atomic Spectrometry, vol. 12, pp. 1255-1261, 1997. 
[26] C. L. Lewis, M. A. Moser, D. E. Dale Jr., W. Hang, C. Hassell, F. L. King, and V. Majidi, "Time-gated pulsed glow discharge: real-time chemical speciation at the elemental, structural, and molecular level for gas chromatography time-offlight mass spectrometry," Analytical Chemistry, vol. 75, no. 9, pp. 1983-1996, 2003.

[27] C. L. Lewis, M. A. Moser, W. Hang, D. E. Dale Jr., D. C. Hassell, and V. Majidi, "Influence of discharge parameters on real-time chemical speciation for gas chromatography pulsed glow discharge plasma time-of-flight mass spectrometry," Journal of Analytical Atomic Spectrometry, vol. 18, pp. 629-636, 2003.

[28] J. C. Wuilloud, R. G. Wuilloud, A. P. Vonderheide, and J. A. Caruso, "Gas chromatography/plasma spectrometry - an important analytical tool for elemental speciation studies," Spectrochimica Acta Part B, vol. 59, pp. 755-792, 2004.

[29] D. Fliegel, R. Waddell, V. Majidi, D. Gunther, and C. L. Lewis, "Quantification of aromatic and halogenated hydrocarbons and alcohol mixtures at the elemental, structural, and parent molecular ion level," Analytical Chemistry, vol. 77, no. 6, pp. 1847-1852, 2005.

[30] D. Fliegel, K. Fuhrer, M. Gonin, and D. Gunther, "Evaluation of a pulsed glow discharge time-of-flight mass spectrometer as a detector for gas chromatography and the influence of the glow discharge source parameters on the information volume in chemical speciation analysis," Analytical Bioanalytical Chemistry, vol. 386, pp. 169-179, 2006.

[31] A. Sola-Vazquez, A. Lara-Gonzalo, J. M. Costa-Fernandez, R. Pereiro, and A. Sanz-Medel, "Gas chromatography coupled to tunable pulsed glow discharge time-of-flight mass spectrometry for environmental analysis," Analyst, vol. 135, pp. 987-993, 2010.

[32] J. Wang, M. Pumera, G. E. Collins, and A. Mulchandani, "Measurements of chemical warfare agent degradation products using an electrophoresis microchip with contactless conductivity detector," Analytical Chemistry, vol. 74, no. 23, pp. 6121-6125, 2002.

[33] R. M. Black and B. Muir, "Derivatisation reactions in the chromatographic analysis of chemical warfare agents and their degradation products," Journal of Chromatography A, vol. 1000, pp. 253-281, 2003. 
[34] J. R. Hancock, P. A. D'Agostino, and L. R. Provost, "Techniques for chemical warfare and mid-spectrum agents," technical report, Defence Research Establishment Suffield, 1999.

[35] C. Schummer, O. Delhomme, B. M. R. Appenzeller, R. Wennig, and M. Millet, "Comparison of MTBSTFA and BSTFA in derivatization reactions of polar compounds prior to GC/MS analysis," Talanta, vol. 77, pp. 1473-1482, 2009.

[36] <http://www.thermo.com/eThermo/CMA/PDFs/Various/File-52881.pdf> accessed 13 July 2013.

[37] <http://www.sigmaaldrich.com/technical-documents/articles/reporter-us/thederivatization.html $>$ accessed 13 July 2013.

[38] T. G. Sobolevsky, A. I. Revelsky, B. Miller, V. Oriedo, E. S. Chernetsova, and I. A. Revelsky, "Comparison of silylation and esterification/acylation procedures in GC-MS analysis of amino acids," Journal of Separation Science, vol. 26, no. 17, pp. 1474-1478, 2003.

[39] T. P. Mawhinney and M. A. Madson, "N-Methyl-N-(tertbutyldimethylsilyl)trifluoroacetamide and related N-tert-butyldimethylsilyl amides as protective silyl donors," Journal of Organic Chemistry, vol. 47, no. 17, pp. 3336-3339, 1982.

[40] M. A. Mohd, ed., Advanced Gas Chromatography - Progress In Agricultural, Biomedical and Industrial Applications. InTech, 2012.

[41] B. P. Masekete, "Derivatisation of alkylphosphonic acids for GC-MS analysis," Master's thesis, Tshwane University of Technology, 2006.

[42] R. E. Steiner, C. L. Lewis, and F. L. King, "Time-of-flight mass spectrometry with a pulsed glow discharge ionization source," Analytical Chemistry, vol. 69, no. 9, pp. 1715-1721, 1997.

[43] L. Li, J. T. Millay, J. P. Turner, and F. L. King, "Millisecond pulsed radio frequency glow discharge time of flight mass spectrometry: temporal and spatial variations in molecular energetics," American Society for Mass Spectrometry, vol. 15, pp. 87-102, 2004.

[44] M. Moser, A. Torrence, M. Graham, A. Wilson, J. Paley, B. Logue, and A. D'Ambrozio, "Novel analytical screening methods for chemical warfare agents 
using solid-phase micro-extraction and gas chromatography mass spectrometry." Personal communication with Matt Moser, 2004. 


\section{Chapter 4}

\section{Application of Pulsed Glow Discharge Chemical Speciation: Explosives}

\subsection{Abstract}

The detection and identification of explosive materials has received large success by combining the separation power of gas chromatography and the specificity of mass spectrometry. In such detection schemes ion sources such as electron ionization or chemical ionization are used to identify different analytes based on either its fragment ions or intact molecular parent ion. Through the combination of these two complementary ionization sources, positive identification is easily achieved. The pulsed glow discharge ion source offers the distinct advantage of producing both fragment and molecular ions within a single analysis; no other technique is capable of providing both pieces of information simultaneously. Thus, using time-gated detection, both fragment and molecular ion spectra can be obtained and give the analyst a greater degree of confidence in the identification of the compound. In this study, the pulsed glow discharge ion source is coupled with gas chromatography to expand its application to the qualitative analysis of explosive materials. Results are presented for nitrobenzene detailing the spectral differences arising from the various temporal glow discharge ion processes. We were able to demonstrate that the pulsed glow discharge provides structural information during the plateau for the analytes and we learned that it was important to control the analyte concentration introduced to the plasma 
so that it does not quench the afterpeak signal. Future directions would focus on lowering the analyte concentration sufficiently.

\subsection{Introduction}

An explosive is defined as a material, either a pure single substance or a mixture of substances, which is capable of producing an explosion by its own energy. With proper initiation, chemical explosives undergo violent decomposition to produce heat, gas, and the rapid expansion of matter. Explosives can be classified as propellants, primary explosives, or high explosives. All explosives (military, commercial, improvised, homemade) require the same ingredients to be considered an explosive; they must contain oxidizer and fuel. Some examples of high explosives are listed in Table 4.1.

Table 4.1: Examples of High Explosives

\begin{tabular}{|c|c|c|}
\hline Nitroarenes & $\mathrm{C}$ ring- $\mathrm{NO}_{2}$ & (TNT, picric acid) \\
\hline Nitroalkanes & $\mathrm{C}-\mathrm{NO}_{2}$ & (nitromethane) \\
\hline Nitrate esters & $\mathrm{O}-\mathrm{NO}_{2}$ & (NG, NC, PETN) \\
\hline Nitramines & $\mathrm{N}-\mathrm{NO}_{2}$ & (RDX, HMX, "plastic") \\
\hline
\end{tabular}

Forensically, the analysis of explosives can identify source materials and evaluate potential threats. The use of explosive materials, especially improvised explosive devices, in the Middle East has increased the urgency for more selective and sensitive detection methods. The standard environmental test method for nitroaromatic, nitramine, and nitroester analysis has been US EPA mehod 8330. 1 This method uses high performance liquid chromatography (HPLC) separation and ultra-violet absorption detection. Because of disadvantages associated with the HPLC method, including high solvent usage, multiple co-elutions, and long analysis times, an alternative method, US EPA method $8095^{2}$ using gas chromatography (GC) with electron capture detection, was developed. Although sensitive for many target explosives, both methods 8330 and 8095 are limited by a lack of detector selectivity and required confirmatory information through the use of a second analytical column.

The application of mass spectrometry (MS) to the analysis of explosives and related compounds dates back to the 1960s. Since that time, MS has become the 
detector of choice for the confirmation of explosive compounds as it offers high levels of sensitivity and specificity compared to other technologies. Its application to the analysis of explosives has been reviewed in several articles and books. ${ }^{3}[5]$ As with any MS detection system, the ionization source is a key component.

Gas chromatography mass spectrometry employing electron ionization is routinely used. Its major strength lies in its ability to identify compounds through the use of extensive mass spectral libraries. However, a number of organic high explosives have analogous structures and fragmentation patterns and the NIST library can prove insufficient for identification. The result is numerous matches with similar match factors and percent probabilities and a reduction in the confidence level of compound identification.

The pulsed glow discharge (GD) plasma is unique in its ability to produce both fragment and molecular ions within a single analysis. Pulsed GD is a dynamic ionization source, meaning that, the pulse cycle is dominated by different ionization processes with respect to time. Thus, the mass spectra acquired with time-gated acquisition are quite different owing to the differences in ionization process that dominate each time regime. From a single pulse cycle, elemental, structural, and molecular information can be generated. Electron ionization dominates the 'prepeak', a mixture of electron ionization and Penning ionization mechanisms occurs during the 'plateau', and Penning ionization is the primary source of ions during the 'afterpeak'. The versatility of pulsed GDs makes them extremely powerful tools for facing new challenges in chemical analysis. This study strives to expand the utility of the pulsed GD ion source with concurrent elemental, structural, and molecular analysis to the field of explosive materials. Results are presented for nitrobenzene detailing the spectral differences arising from the various temporal glow discharge ion processes. Nitrobenzene by itself is not considered explosive. However, nitrobenzene acts as a fuel and forms an explosive mixture when oxidizers, such as nitrates and chlorates, are added $[\sqrt[6]{9}$ Additionally, nitrobenzene is an explosives target compound found in the calibration mixes used in United States EPA Method 8330 and Method 8095.112

\subsection{Materials and methods}

\subsubsection{Materials}

A standard solution of nitrobenzene, $1000 \mu \mathrm{g} / \mathrm{mL}$ in acetonitrile (Supelco) was used in this study. Nitrobenzene, b.p. $210{ }^{\circ} \mathrm{C}$, standards were stored in a freezer and were 
allowed to warm to room temperature before use.

\subsubsection{Instrumentation}

Samples were introduced into the GC (Hewlett Packard 5890 Series II) by manual injection of the sample, $1-2 \mu \mathrm{L}$, using a gas-tight syringe into the split injector of a GC equipped with a J\&W Scientific DB-1MS, (1\%-Phenyl)-methylpolysiloxane, fused silica capillary column. The GC is temperature-programmed to separate the analytes which are detected by GD-TOFMS. The interfacing of GC with GD requires that the effluent from the GC column be transported to the inlet of the torch. The main requirement of the GC to GD interface is that the volatilized analytes remain in the gas phase as they travel from the GC column to the plasma. Therefore, any condensation of the analytes should be avoided to assure not only minimal loss of analyte but also optimal peak resolution. This can be achieved by heating the transfer line to accommodate the volatility of the analyte species.

The configuration of the dc GD ion source along with the sample introduction system and direct insertion probe used in this work is shown in Figure 4.1. The glow discharge chamber consists of a stainless steel six-way cross, fitted with conflat flanges. The copper cathode is mounted onto the dc direct insertion probe and introduced into the glow discharge chamber (which also served as the anode) through a ball valve fitted with a $1 / 2$ inch adapter that allows the probe to be inserted without breaking the vacuum.

The $5 \mathrm{~mm}$ diameter copper cathode is surrounded by a macor shield (ceramic insulator). This insulator has the effect of localizing the plasma onto the flat surface of the cathode. A square-wave dc pulse was applied to the cathode which was pulsed between ground and a high negative potential. A digital delay generator (DDG) (Model DG535, Stanford Research Systems, Inc.) controlled the pulse duration and frequency by gating an electronic pulser (Model PVX-4140, DEI) that converted dc power (Model PS350, Stanford Research Systems, Inc.) into a series of high voltage pulses. All electronic parameters were remotely controlled using LabVIEW software (LabVIEW 7.1, National Instruments) developed in-house. The GD chamber was filled with ultra high purity grade argon gas (Scott Specialty Gases) at an operating pressure of 0.50 Torr as monitored by a thermocouple gauge (Active gauge, Edwards).

The ion beam from the GD source was oriented orthogonally to the time-offlight mass spectrometer. The TOF used in this study was designed and constructed in house, Figure 4.1. Argon and sputtered species exit the glow discharge chamber 


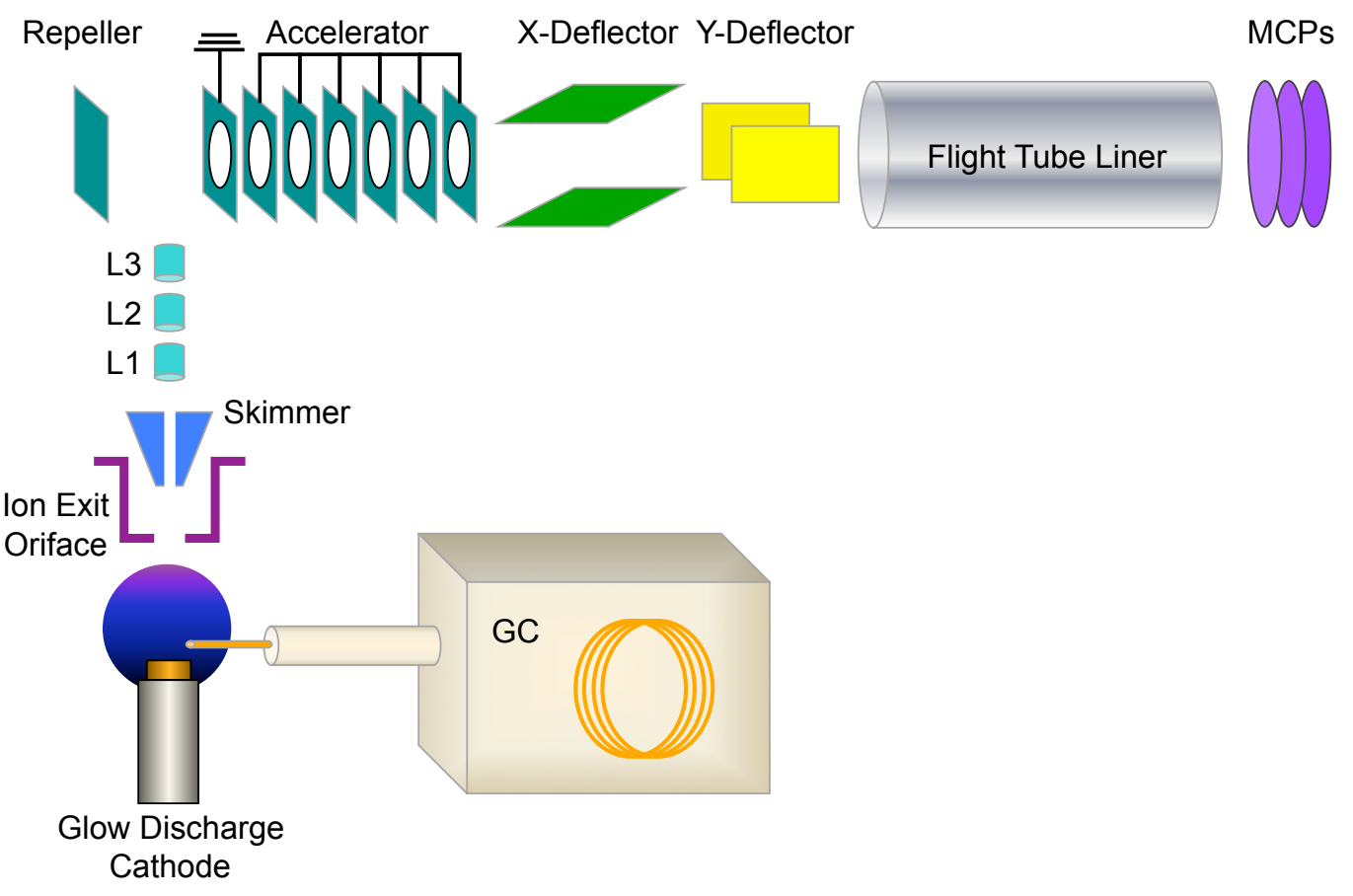

Figure 4.1: Gas chromatography glow discharge time-of-flight mass spectrometer. Adapted from reference. 10 
Table 4.2: GC/Pulsed GD-TOFMS Operating Conditions

\begin{tabular}{ll}
\hline Gas Chromatograph & \\
\hline Stationary phase & J\&W DB-1MS \\
Column dimensions & $30 \mathrm{~m} \mathrm{x} 0.25 \mathrm{~mm}$ i.d. \\
Film thickness & $0.50 \mu \mathrm{m}$ \\
Carrier gas & Argon \\
Injector temp & $250{ }^{\circ} \mathrm{C}$ \\
Initial temperature & $50{ }^{\circ} \mathrm{C}(2 \mathrm{~min})$ \\
Ramp rate & $15{ }^{\circ} \mathrm{C} / \mathrm{min}$ \\
Final temperature & $100{ }^{\circ} \mathrm{C}(10 \mathrm{~min})$ \\
\hline Pulsed Glow Discharge & \\
\hline Cathode material & $\mathrm{copper}$ \\
Pressure: discharge gas (Ar) & $0.50 \mathrm{Torr}$ \\
Pulse voltage & $-1100-1200 \mathrm{~V}$ \\
Pulse width & $5.0 \mathrm{~ms}$ \\
Pulse frequency & $100 \mathrm{~Hz}$ \\
\hline Time-of-Flight Mass Analyzer & \\
\hline Pressure: 1st vacuum stage & $10^{-3} \mathrm{Torr}$ \\
Pressure: 2nd vacuum stage & $10^{-6} \mathrm{Torr}$ \\
Sampling orifice diameter & $1 \mathrm{~mm}$ \\
Skimmer orifice diameter & $1 \mathrm{~mm}$ \\
Entrance slit width & $2.5 \mathrm{~mm}$ \\
Repeller pulse width & $2 \mu \mathrm{s}$ \\
Flight tube length & $1.0 \mathrm{~m}$ \\
Potential: skimmer & $-580 \mathrm{~V}$ \\
Potential: slit & ground \\
Potential: L1 & $-150 \mathrm{~V}$ \\
Potential: L2 & $-600 \mathrm{~V}$ \\
Potential: L3 & $-480 \mathrm{~V}$ \\
Potential: repeller & $+140 \mathrm{~V}$ \\
Potential: repeller bias & $-5 \mathrm{~V}$ \\
Potential: extractor grid & ground \\
Potential: accelerator / flight tube & $-2110 \mathrm{~V}$ \\
Potential: X steering plate & $-2200 \mathrm{~V}$ \\
Potential: Y steering plate & $-21400 \mathrm{~V}$ \\
Potential: micro-channel plate detector & $-2000 \mathrm{~V}$ \\
\hline &
\end{tabular}


through an ion exit orifice ( $1 \mathrm{~mm}$ i.d.) and enter the skimmer cone $(1 \mathrm{~mm}$ i.d.) which selects a portion of the beam for transmission to the ion optics of the high-vacuum segment of the mass spectrometer. Ions transmitted through the skimmer cone were focused without changing the ion energies into the repeller region of the mass analyzer by means of a three-tube Einzel lens assembly and ion slit $(2.5 \mathrm{~mm}$, fixed at ground potential). A second DDG (Model 9650A, Signal Recovery), synchronized to the DDG of the pulsed GD power supply, gated a second electronic pulser and dc power supply to provide the TOF repeller pulse. The repeller plate was pulsed $2 \mu \mathrm{s}$ with a positive potential to orthogonally inject a packet of ions from the GD pulse cycle into the flight tube. Adjustment of the gate delay position was accomplished using the repeller plate's DDG. By delaying the $2 \mu$ s repeller pulse with respect to the initiation of the ms cathodic pulse, different portions of the GD pulse cycle could be sampled. The lens stack used for ion acceleration was constructed using a series of 2 inch square stainless steel plates each with a 1 inch diameter grid (Kimball Physics). General operating conditions for the GD-TOF mass spectrometer balancing ion transmission and resolution are listed in Table 4.2 .

\subsubsection{Data collection}

Nearly concurrent data collection of three GD time regimes was accomplished using a pair of digital delay generators and a set of three oscilloscopes (Waverunner LT372, LeCroy). The first digital delay generator, A, controlled the glow discharge voltage frequency and pulse width as well as externally triggered the second digital delay generator, B, so that they were in sync. The second digital delay generator, B, controlled the three delay gates for initiating the repeller pulse. Each repeller pulse was $2 \mu \mathrm{s}$ in duration and all three were tied together to drive the repeller power supply of the TOFMS. Each oscilloscope was triggered by a different digital delay pulse and the signal from the TOF's microchannel plate detector was split into each oscilloscope, thus, providing three separate observation windows from a single GD pulse. An illustration of this configuration is shown in Figure 4.2. Data from each oscilloscope was acquired onto a computer via a LabVIEW program developed inhouse. A second LabVIEW program was used for data reduction and interpretation purposes. The data shown in the prepeak spectra were acquired at $6 \mu$ s after pulse ignition, the plateau at $4 \mathrm{~ms}$ after pulse ignition, and the afterpeak at $0.6 \mathrm{~ms}$ after pulse termination. 


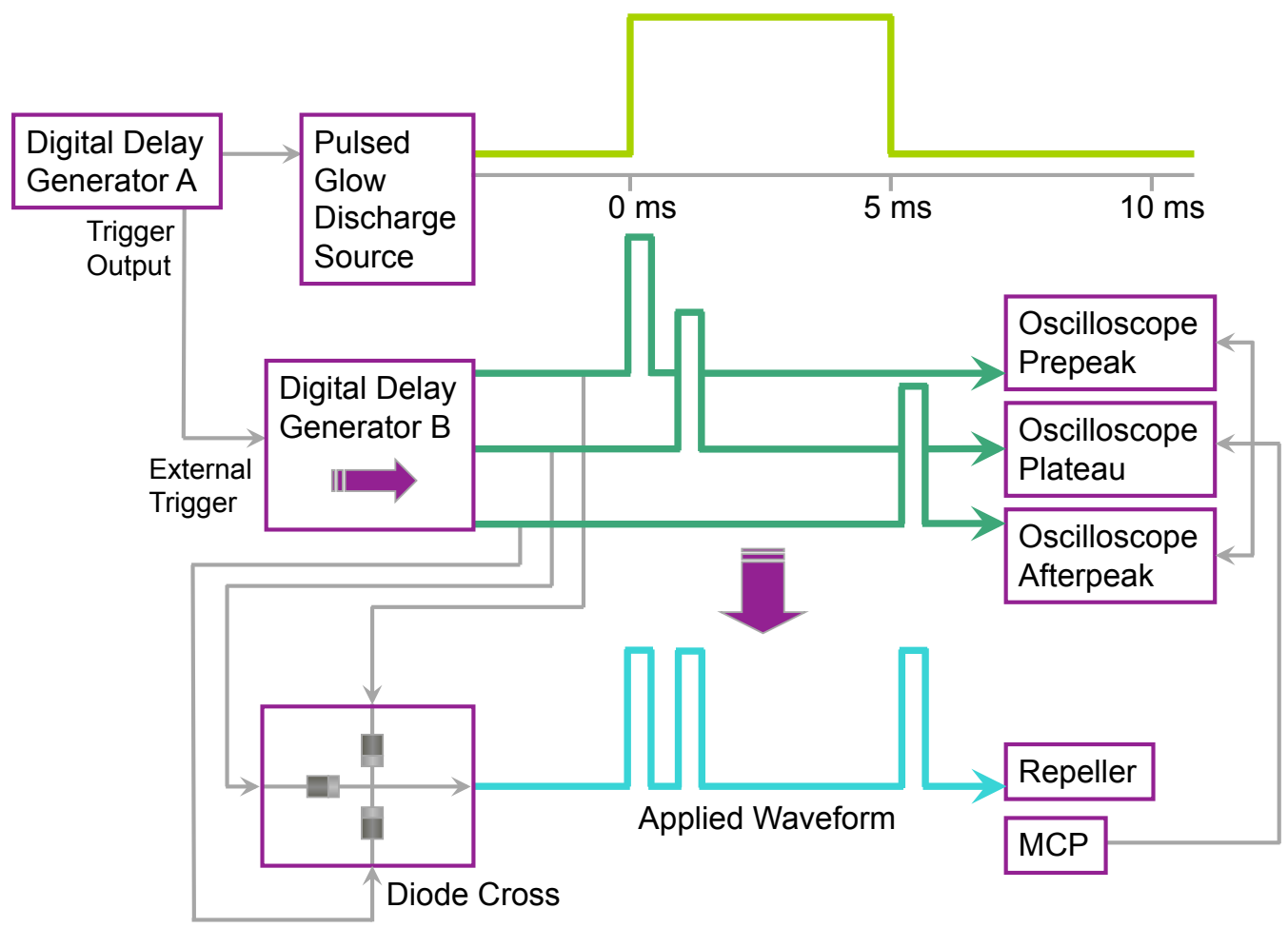

Figure 4.2: Time gated acquisition of mass spectra from three different temporal regimes of a single glow discharge pulse. Adapted from reference.10 


\subsection{Results and discussion}

In 1999, Steiner et al. 11 demonstrated the concurrent acquisition of elemental and molecular information for the compound $p$-xylene, introduced through a leak valve, using time-gated pulsed GD-TOFMS. In 2000, Majidi et al. ${ }^{12}$ used the same method in the analysis of 'thermometer molecules' to calculate the approximate ionization energy available in the different discharge time regimes. However, the use of a leak valve often lead to chaotic plasma behavior. In 2003, Lewis et al. ${ }^{[10 \mid 13}$ first reported on the coupling of GC to this exciting new method, GC/GD-TOFMS, for the separation and chemical speciation of common aromatic and halogen compounds.

The expansion of this method to the analysis of real world samples, such as a mixture of explosive compounds, is the focus of this study. Data was acquired concurrently from the prepeak, plateau, and afterpeak time regimes of a pulsed GD ion source in order to obtain elemental-, structural-, and molecular-based mass spectra using a GC/GD-TOFMS. Preliminary results are shown for nitrobenzene demonstrating the ability to concurrently acquire mass spectra from different time regimes of a pulsed GD plasma.

Nitrobenzene eluted the gas chromatograph with a retention time of 3.5 minutes. Figure 4.3 illustrates pulsed GD-TOFMS mass spectra obtained during the analysis of nitrobenzene. Data from the prepeak, plateau, and afterpeak were collected for the sample. Figure 4.4 depicts the background-subtracted mass spectra. It is important to note that background-subtracted mass spectra for GC is, in most situations, performed using commercial software with peaks in centroid format. The background subtracted spectra shown in Figure 4.4 were created using LabVIEW software with peaks in profile format.

Compounds were identified by comparing the mass spectra obtained with those stored in the National Institute of Standards and Technology (NIST) library. Sampling ions during the plateau produced a fragmentation pattern resembling that of the NIST reference spectrum obtained with conventional $70 \mathrm{eV}$ electron ionization (not pictured). The characteristic ions for nitrobenzene, $m / z$ 77, 93, 107 and 123, can been seen during the plateau of the pulsed GD. The major ions are the nitrobenzene molecular ion at $m / z 123\left(\mathrm{M}^{+}\right)$, and a series of fragment ions, $\mathrm{C}_{6} \mathrm{H}_{5}^{+}$at $m / z$ $77, \mathrm{C}_{6} \mathrm{H}_{5} \mathrm{O}^{+}$at $m / z 93$, and $\mathrm{C}_{6} \mathrm{H}_{5} \mathrm{NO}^{+}$at $m / z 107$.

The afterpeak time regime showed no ions at all in the mass spectrum. The afterpeak is known to be dominated by Penning ionization. With metastable potential energies of 11.55 and $11.72 \mathrm{eV}$, argon metastables are capable of ionizing most 


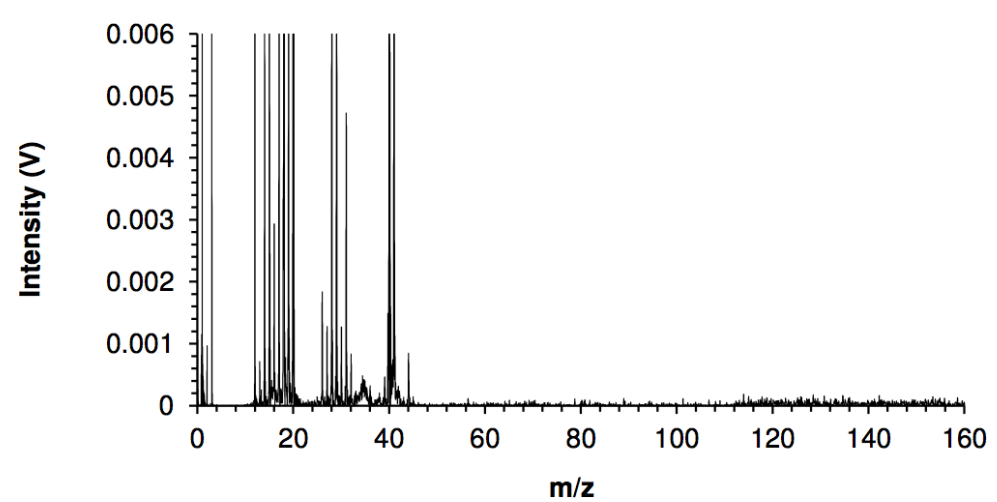

(a) Prepeak

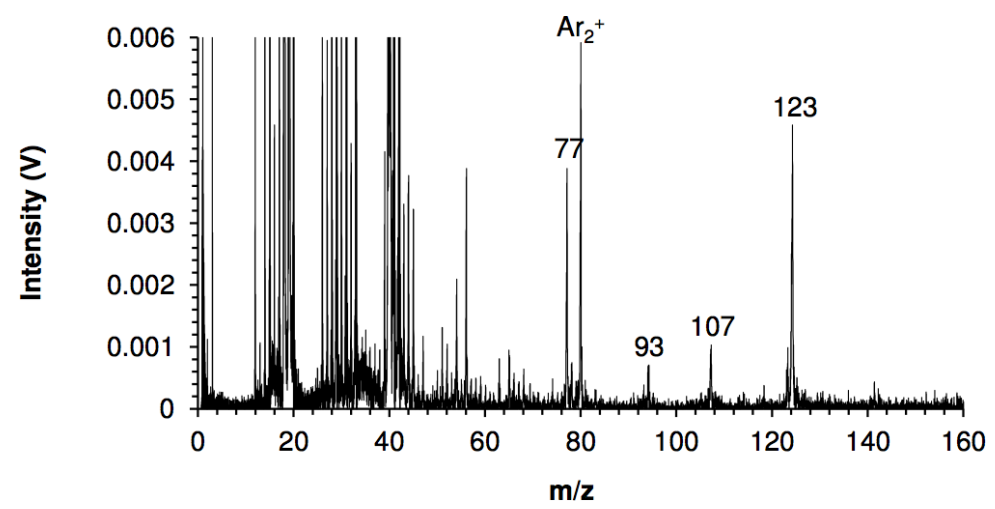

(b) Plateau

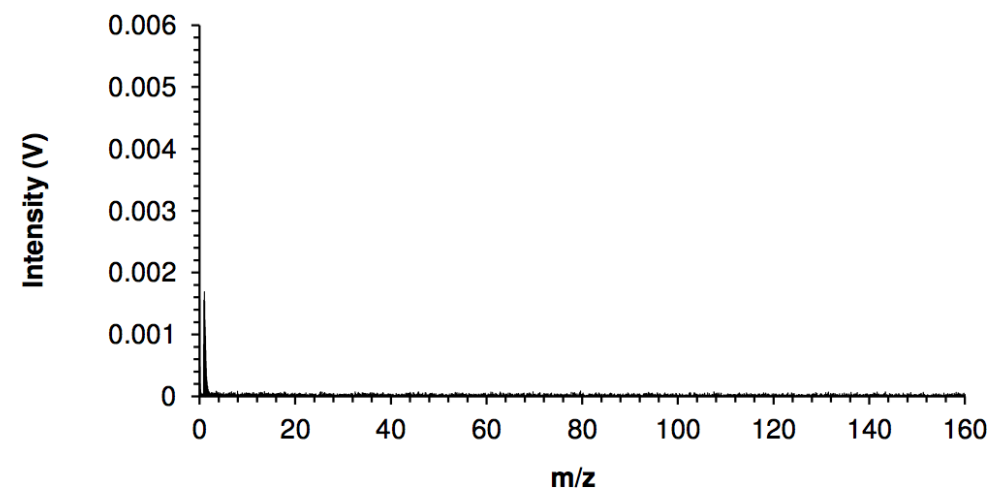

(c) Afterpeak

Figure 4.3: Mass spectra of nitrobenzene produced using pulsed GDMS with timegated acquisition from three different temporal regimes. $1000 \mu \mathrm{g} / \mathrm{mL}$ nitrobenzene, $1 \mu \mathrm{L}$ volume. 


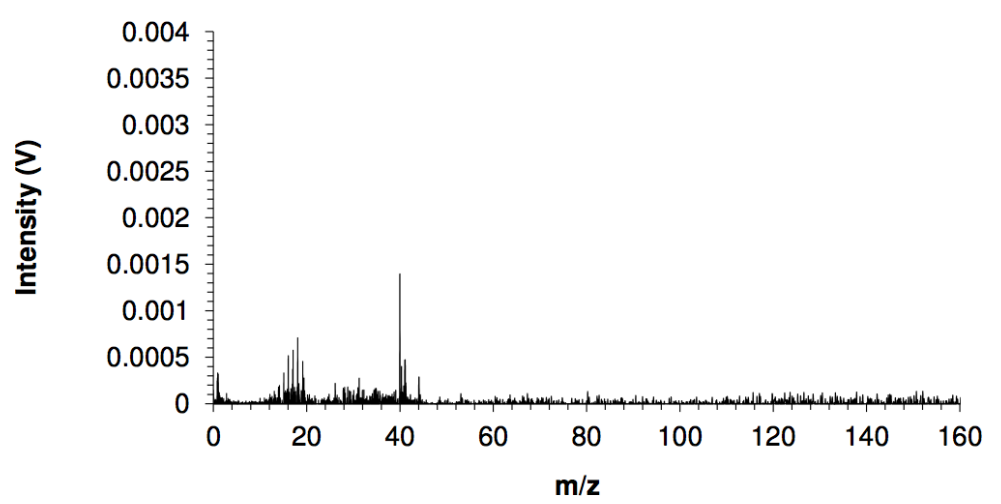

(a) Prepeak

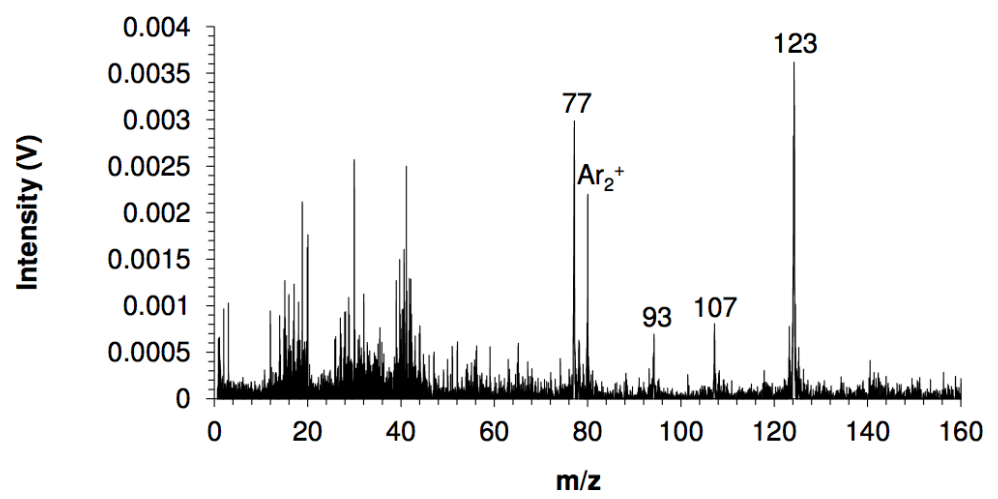

(b) Plateau

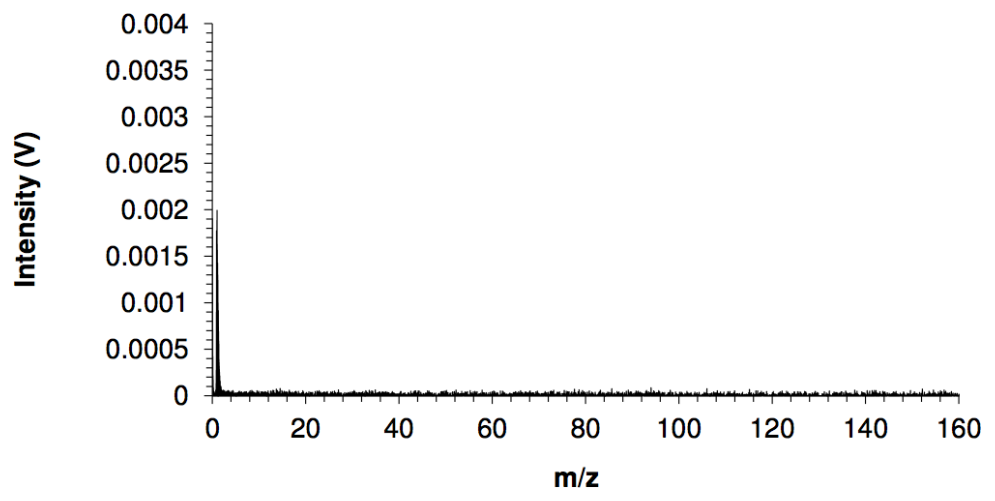

(c) Afterpeak

Figure 4.4: Background-subtracted mass spectra of nitrobenzene produced using pulsed GDMS with time-gated acquisition from three different temporal regimes. $1000 \mu \mathrm{g} / \mathrm{mL}$ nitrobenzene, $1 \mu \mathrm{L}$ volume. 
molecules. At first look, the absence of ions in the afterpeak could indicate that the ionization potential of the molecule is above that of the argon metastable energies. But upon closer inspection, the ions from the copper cathode are not present. Furthermore, the ionization potential of the test molecule, nitrobenzene, is approximately $9.94 \mathrm{eV}{ }^{14}$ This indicates that the plasma was quenched during this time regime.

It is known that increased analyte concentration in the discharge quenches the Ar metastable population, especially in the afterpeak. Quenching of the plasma was consistently an issue and as such the distance between the GC capillary column and the cathode was increased. Further adjustments were made to reduce sample concentration load on the plasma by increasing the split ratio using the total flow controller knob. However, the total ion chromatogram, monitored using LabVIEW, showed no significant change as the split ratio was manually adjusted. It was therefore concluded that the total flow controller (split valve) on the GC was not functioning properly.

\subsection{Conclusions and future directions}

The pulsed GD-TOFMS as a detector for GC separations showed promise for the chemical speciation of explosive materials in real time. Preliminary results for the test molecule, nitrobenzene, show mass spectra acquired during the plateau have the molecular ion and a similar fragmentation pattern as that of the NIST reference spectrum. Unfortunately, preliminary data was unable to illustrate the additional benefits of concurrently acquiring mass spectra from the prepeak or afterpeak and the study was prematurely ended when the GC's split valve was found to be not functioning.

Use of instrumentation that is within specification, working correctly, and adequately calibrated is fundamental to the method development process. In order to improve the GC/GD-TOFMS technique, chromatography concerns would first need addressed.

Following successful GC operation, the different GD source parameters like capillary distance, cathode-anode spacing, and GD source pressure with regards to the accessible elemental, structural, and molecular information would need to be evaluated for method optimization. An explosive compound test mix, such as an 8095 Calibration Mix (Restek, Bellefonte, PA), could be used to assess system performance. 


\section{Bibliography}

[1] <http://www.epa.gov/epaoswer/hazwaste/test/pdfs/8330a.pdf> accessed 14 July 2013.

[2] <http://www.epa.gov/epaoswer/hazwaste/test/pdfs/8095.pdf> accessed 14 July 2013.

[3] J. Yinon and S. Zitrin, Modern methods and applications in analysis of explosives. John Wiley \& Sons, Ltd., 1993.

[4] M. E. Sigman and C. Y. Ma, "Detection limits for GC/MS analysis of organic explosives," Journal of Forensic Science, vol. 46, pp. 6-11, 2001.

[5] D. S. Moore, "Instrumentation for trace detection of high explosives," Review of Scientific Instruments, vol. 75, no. 8, pp. 2499-2512, 2004.

[6] C. M. Mason, R. W. Van Dolah, and J. Ribovich, "Detonability of the system nitrobenzene, nitric acid, and water," technical report, Explosives Research Laboratory, Bureau of Mines, U.S. Department of the Interior, Pittsburgh Pennsylvania, 1961.

[7] <http://www.caledonlabs.com/upload/msds/5470-5e.pdf> accessed 14 July 2013.

[8] <http://msds.dupont.com/msds/pdfs/EN/PEN09004a2f800069fb.pdf> accessed 14 July 2013.

[9] <http://www.fishersci.in/msds/Nitrobenzene.pdf> accessed 14 July 2013.

[10] C. L. Lewis, M. A. Moser, D. E. Dale Jr., W. Hang, C. Hassell, F. L. King, and V. Majidi, "Time-gated pulsed glow discharge: real-time chemical speciation at the elemental, structural, and molecular level for gas chromatography time-offlight mass spectrometry," Analytical Chemistry, vol. 75, no. 9, pp. 1983-1996, 2003.

[11] R. E. Steiner, C. L. Lewis, and V. Majidi, "Consideration of a millisecond pulsed glow discharge time-of-flight mass spectrometer for concurrent elemental and molecular analysis," Journal of Analytical Atomic Spectrometry, vol. 14, pp. 1537-1541, 1999. 
[12] V. Majidi, M. A. Moser, C. L. Lewis, W. Hang, and F. L. King, "Explicit chemical speciation by microsecond pulsed glow discharge time-of-flight mass spectrometry: concurrent acquisition of structural, molecular and elemental information," Journal of Analytical Atomic Spectrometry, vol. 15, no. 1, pp. 19-25, 2000 .

[13] C. L. Lewis, M. A. Moser, W. Hang, D. E. Dale Jr., D. C. Hassell, and V. Majidi, "Influence of discharge parameters on real-time chemical speciation for gas chromatography pulsed glow discharge plasma time-of-flight mass spectrometry," Journal of Analytical Atomic Spectrometry, vol. 18, pp. 629-636, 2003.

[14] <http://webbook.nist.gov/chemistry> accessed 22 September 2006. 


\section{Chapter 5}

\section{Measuring Intrinsic Molecular Properties of Microbial Particles for Attribution Purposes}

\subsection{Abstract}

Various techniques are used to determine physical and chemical properties of Bacillus globigii spores, a Bacillus anthracis surrogate, for attribution purposes. In response to the need for fingerprint libraries of biological signatures, particle characteristics such as shape, surface morphology, and molecular vibrations as they relate to different size fractions, were investigated. It was anticipated that by first separating the sample into different class sizes, the size fractions would exhibit additional signatures that were previously undetectable in the bulk sample. A variety of techniques were employed in the analysis, including gas displacement pycnometry, aerodynamic particle sizing, scanning electron microscopy, and Raman spectroscopy. It was found that size separation permitted a more rapid determination by SEM to confirm the presence of spores, but did not enhance the ability of Raman to identify the spores. Ultimately, results from these analyses can be used to build a library to determine an organism's unique biological signature that can be correlated with known growth and processing methods to identify how, when, and where the sample was produced. 


\subsection{Introduction}

Although they are invisible to us, microbes have a huge influence on our lives. And while most microbes are completely harmless, others can be detrimental to our health - causing diseases like anthrax. Potentially pathogenic microbes can be classified into four major groups: viruses, bacteria, fungi, and protozoa.

Bacillus anthracis (BA) (the causative agent for anthrax) is a Gram-positive and rod-shaped bacteria. Bacteria belonging to the genus Bacillus are spore-forming; during adverse conditions, BA produces spores - a hard wall surrounding the cell for survival. ${ }^{1]} \mathrm{BA}$ spores originate in grass and soil, infecting animals who graze in contaminated areas. Humans can contract the disease through contact with infected animals, their hides, or meat; anthrax is not passed from person-to-person. Humans become infected in one of three portals of entry: cutaneous anthrax, gastrointestinal anthrax, and inhalation anthrax (the most deadly form). ${ }^{2}$ Once in the body, the spores return to their bacteria state, followed by a period of reproductive growth. As their numbers increase, the developing anthrax infection often eventually overcomes the immune system and releases deadly toxins that interfere with bodily functions.

Bioterrorist attacks can involve the intentional release of bacteria against humans for hostile purposes. In the years following the 2001 deadly mailings of Bacillus anthracis spores, a wave of detection and identification methods for biological weapons have been developed. These analytical methods include: DNA- $-\frac{315}{5}$ or antibody-based assays,, 617 mass spectrometry (MS)-based proteomics,,$[12$ and several non-proteomics MS methods. ${ }^{13}-16$ But much more can be learned from the detailed analysis of illicit microbial samples than simply "What is it?" There are multiple direct detection techniques that can instead focus on the microbial material's intrinsic properties (shape, elemental composition, vibrational modes), which can fingerprint the nonDNA molecule itself. The manufacturing of biological agents often uses inorganic chemicals or components in many of the growth and post-growth processing steps, leaving behind signatures as to the material's origin.

Attribution is defined as "the ascribing of a work to a particular author or artist." 17$]$ For microbial forensic purposes, the ultimate goal of attribution is to find evidence that will link a bioagent to the perpetrator responsible for a biological attack. The detailed characterization of a biological material is crucial for successful attribution and prosecution $\frac{18-22}{22}$ and involves many analytical tools.

To aid the attribution effort, one needs to collect information on the particulate material's physical, morphological, chemical, and isotopic characteristics. For 
instance, spores can possess distinct shapes and sizes. Both the overall shape and the detailed surface features can provide signatures that are best measured with high resolution images of the sample from techniques such as atomic force microscopy ${ }^{23}$ or scanning electron microscopy (SEM). Vibrations at bonds between atoms comprising the molecules can produce unique characteristic spectra. To this end, Raman spectroscopy is a fast and nondestructive analysis method that has proven to be successful in the study of bacterial spores. $\frac{24}{27}$ It is capable of distinguishing between dormant spores and vegetative bacterial cells, $\frac{28}{28}$ and identifying bacterial species ${ }^{29}$ or strains. Elemental and isotopic compositions, determined by inductively coupled mass spectrometry (ICP-MS), can be used as an inorganic chemical fingerprint. ${ }^{[13}$ Schaldach et al. ${ }^{21}$ analyzed bacterial spores to develop a database of non-DNA based signatures using a variety of analytical techniques: SEM with energy dispersive X-ray microanalysis, atomic force microscopy, surface-enhanced Raman spectroscopy, bio-aerosol time-of-flight mass spectrometry, time-of-flight secondary ion mass spectrometry, accelerator mass spectrometry, particle-induced X-ray emission, and scanning transmission ion microscopy. Because the analysis of a bulk sample results in data that averages out many of the differences between individual cells, much of their work ${ }^{21}$ was done at the single-spore level, requiring samples to be grown in a systematic and homogenous manner.

Here we present a preliminary study of a number of different methodologies to probe for biological signatures within size-fractionated samples of Bacillus globigii spores, a nonpathogenic surrogate for Bacillus anthracis. The list of analytical techniques is not exhaustive (as in any single location, availability of instrumentation is limited), however it is enough to illustrate the benefits of size-fractionating a particulate sample prior to analysis. It is believed that certain signatures can be detected within certain size fractions that would otherwise be masked in bulk analysis of the powder. Because the analysis does not involve individual spores, rigorous control of growth conditions and homogeneity is unnecessary. The powder sample was sizefractionated using a micro-orifice uniform deposit impactor (MOUDI) and then the fractions were subsequently analyzed for morphology and true particle size using scanning electron microscopy and for functional groups using Raman spectroscopy. In addition, a pycnometer determined particle density and an aerodynamic particle sizer (APS) identified the particulates aerodynamic size distribution. The combination of these complimentary techniques are essential for investigating signatures of microbial materials relating to sample growth, processing, and geographic location.

Bacillus globigii (BG) served as a surrogate for the potential bioterrorism agent Bacillus anthracis, an anthrax-causing bacterium. BG is a spore forming microor- 
ganism whose spores share a similar size, shape, and surface morphology as BA, but are nonlethal. The CDC lists BG as a "Class 1" organism, meaning it presents no recognized hazards. Because of its harmless nature and similarities to BA, BG has found widespread use in the research field as a biowarfare simulant. $.30+34$

\subsection{Materials and methods}

\subsubsection{Materials}

Polystyrene DVB (density $1.05 \mathrm{~g} / \mathrm{cm}^{3}$ ) microspheres $5.1 \mu \mathrm{m} \pm 0.4 \mu \mathrm{m}$, aluminum oxide (density, $3.97 \mathrm{~g} / \mathrm{cm}^{3}$ ) particles $0.5-20 \mathrm{\mu m}$, and aluminum silicate (density, 2.60 $\mathrm{g} / \mathrm{cm}^{3}$ ) particles $0.2-8.0 \mu \mathrm{m}$ were purchased from Duke Scientific. Bacillus globigii in spore form was stored in a desiccator containing silica gel under refrigeration at 4 ${ }^{\circ} \mathrm{C}$ until needed for experimentation. The origin of the sample was unknown.

\subsubsection{Pycnometer}

To analyze the density of the samples, a $1 \mathrm{~cm}^{3}$ AccuPyc 1330 Pycnometer (Micromeritics Instrument Corp.) was employed. The AccuPyc 1330 uses helium gas displacement to measure sample volume and incorporates an external measurement of sample mass to calculate sample density. The pycnometer was first calibrated using a known volume standard preceding each sample analysis. Each aliquot (0.3-0.5 g) of the sample was weighed in the $1 \mathrm{~cm}^{3}$ sample cup and then both were placed inside the pycnometer. The AccuPyc 1330 determined volume a total of five consecutive times and an average density was then recorded. Six aliquots of the same BG sample were analyzed in the gas pycnometer to minimize random density variations due to sampling. Because pycnometry is a non-destructive technique, the sample was reclaimed and used in further analyses.

\subsubsection{Particle size distribution analyzer}

An APS (Model 3320, TSI Inc., St Paul, MN) used in conjunction with a small scale powder disperser (SSPD, Model 3433, TSI Inc., St Paul, MN) was used to collect particle size data on the Bacillus spores. The APS 3320 sizes particles in the range of 0.5 to 20 micrometers in aerodynamic diameter using a time-of-flight measurement 
between two slightly overlapping laser beams. Particles are counted by the APS as they pass individually through the measurement zone resulting in number-weighted size distributions. Data management software provided by TSI (Aerosol Instrument Manager, TSI) converted the number-weighted size distribution to a mass-weighted size distribution based on density measurements from the pycnometer. Reported values represent the mean based on 5 replicate measurements each with a sample time of $300 \mathrm{~s}$.

\subsubsection{Particle size fractionation}

Aliquots of Bacillus globigii spores were aerosolized by the SSPD and then sizeseparated and collected by a commercially available ten-stage rotating micro-orifice uniform deposit impactor (MOUDI, Model 110, MSP Corp, Minneapolis, MN), described in more detail by Marple et al ${ }^{35}$ Silicone tubing, 0.5 inch, directed the particle stream from the outlet of the SSPD to the sampling inlet of the MOUDI. The MOUDI consisted of ten removable impaction stages plus an inlet and an after-filter located in the base. A ten-stage impactor plus inlet included aerodynamic diameter cut-points of $18,10.0,5.6,3.2,1.8,1.00,0.56,0.32,0.18,0.100$, and $0.056 \mu \mathrm{m}$. The aforementioned nominal cut-points hold true at standard atmospheric temperature and pressure, ${ }^{35}$ but this work was performed in Los Alamos, NM, an area of considerable lower atmospheric pressure. For a fixed inlet volumetric flow rate of 30 $\mathrm{L} / \mathrm{min}$ at $20^{\circ} \mathrm{C}$ and a reduced pressure of $0.75 \mathrm{~atm}$, the MOUDI's cut-points were estimated: ${ }^{[36} 17.7,9.8,6.2,3.1,1.8,1.0,0.54,0.30,0.16,0.082$, and $0.044 \mu \mathrm{m}$.

Within the MOUDI, each impaction stage was equipped with an uncoated aluminum foil substrate, $47 \mathrm{~mm}$ in diameter (MSP Corp) and the base with a PTFE after-filter, $37 \mathrm{~mm}$ in diameter (Pall Life Sciences). The removable impaction substrates collected particles, aerosolized by the SSPD and fractionated by the MOUDI, and were subsequently analyzed by gravimetry to identify their mass concentration. These substrates were weighed three times prior to and following sampling using a microbalance (MX5, Mettler-Toledo Inc, Hightstown, NJ) with a precision of $1 \mathrm{\mu g}$. The weightings were averaged and the differences were calculated to determine the amount of material impacted onto each stage. Reported values for samples represent the mean based on 5 replicate measurements. Experimental blanks were used for quality assurance and were run in triplicate prior to sample runs. 


\subsubsection{Scanning electron microscope}

High-resolution imaging and surface characterization of the spore sample were performed with an Inspect $\mathrm{F}$ field emission gun (FEG) scanning electron microscope from FEI Company. The MOUDI aluminum foil substrates with impacted BG particulate were cut down to size (approximately $10 \mathrm{~mm}$ ) and attached to carbon tape. Low beam energies $(1.00 \mathrm{kV})$ gave images of the outer surface detail. Using an FEGSEM operated at low voltage allowed for the nonconducting specimens to be imaged without conductive coatings.

\subsubsection{Raman spectrometer}

The MOUDI fractionated samples were analyzed by a portable Raman spectrometer (R-3000, Raman Systems, Austin, TX) equipped with a $250 \mathrm{~mW} 785 \mathrm{~nm}$ (red) solidstate diode laser with a spectral range of $200-2700 \mathrm{~cm}^{-1}$ and a spectral resolution of $10 \mathrm{~cm}^{-1}$. The beam spot size of the laser was $100 \mu \mathrm{m}$ in diameter. The signal was integrated for 4 minutes for each spectrum and at least three spectra were taken of each sample. The performance of the Raman system was first verified using spectroscopic standard poly-tetra-fluoroethylene (Teflon) prior to each analysis.

\subsection{Results and discussion}

\subsubsection{Density}

Density is a key property for obtaining a particle's aerodynamic diameter as determined by an APS. The measured densities for the Bacillus globigii sample are shown in Table 5.1. The table shows densities for each aliquot on a $\mathrm{g} / \mathrm{cm}^{3}$ basis and the standard deviation. The six aliquots of BG spores exhibited an average density of $1.4242 \mathrm{~g} / \mathrm{cm}^{3}$.

\subsubsection{Particle size distribution}

When sprayed as a fine aerosol mist, anthrax could be a weapon of mass destruction. Fortunately, 'weaponization' of B. anthracis can be difficult. The organism is grown in the lab and when removed from a nutrient-rich environment, the bacteria turns into spores, which naturally clump together. To inhibit clumping, post growth 
Table 5.1: Pycnometer measurements for Bacillus globigii particulate sample.

\begin{tabular}{ccc}
\hline Aliquot & Avg Density $\left(\mathrm{g} / \mathrm{cm}^{3}\right)$ & Std Dev \\
\hline 1 & 1.4214 & 0.0009 \\
2 & 1.4270 & 0.0017 \\
3 & 1.4225 & 0.0023 \\
4 & 1.4222 & 0.0014 \\
5 & 1.4217 & 0.0010 \\
6 & 1.4302 & 0.0003 \\
\hline
\end{tabular}

processing of anthrax spores is done for 'weaponization.' In this context, the term 'weaponization' is a refinement process that combines the spores with fine particles to maintain separation and increase the time they can suspend in the air, making them more respirable and able to do more damage.

Particle size analysis is a major parameter in determining airborne behavior. More specifically, the aerodynamic diameter of an aerosol determines where and how efficiently the particles will deposit in the respiratory tract. Aerodynamic sizing of particles is a technique widely used to obtain high-resolution size distributions of particles on the micrometer scale. An APS measures aerodynamic diameter, a function of both physical size and density, and an important parameter for studying the respiration of particles. ${ }^{37}$ In comparison with cascade impactors, APSs are faster (as short as seconds for a complete measurement) and provide significantly better size resolution (52 size channels). Yet the sample is consumed and not available for subsequent analysis.

As measured by the APS, Figure 5.1, the average number-weighted aerodynamic diameter of the BG spore sample was $0.835 \mu \mathrm{m}$. Individual BG spores are known to have an aerodynamic size of approximately $0.9 \mu \mathrm{m} . \underline{38}$

\subsubsection{APS comparison with MOUDI cascade impactor}

The performances of the APS and MOUDI were evaluated in a controlled study. To cover the range of MOUDI cut sizes three different particle size standards were employed: polystyrene DVB microspheres $(5.1 \mu \mathrm{m} \pm 0.4 \mu \mathrm{m})$, aluminum oxide particles $(0.5-20 \mu \mathrm{m})$, and aluminum silicate particles $(0.2-8.0 \mu \mathrm{m})$. A comparison of APS and cascade impactor measurements of the aforementioned test samples was conducted. 


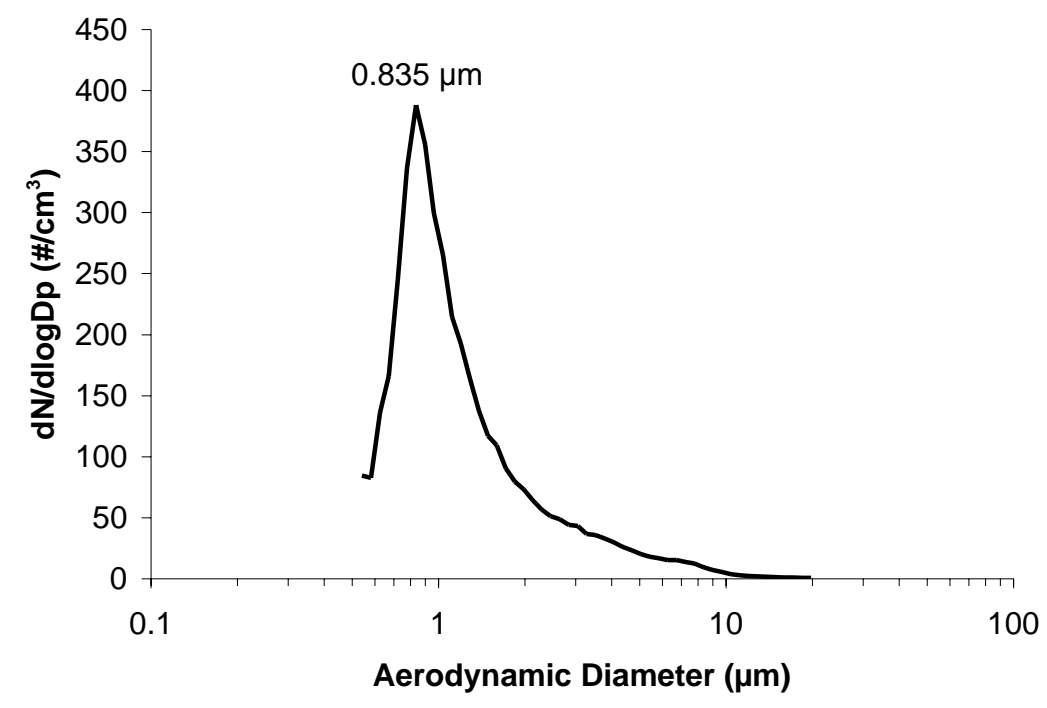

Figure 5.1: Average APS number-weighted size distribution for BG spores. 
Average mass-weighted size distribution measurements from APS measurements were compared to those obtained from MOUDI gravimetric analysis as shown in Figures 5.2. 5.3, and 5.4. A comparison of aerodynamic diameters measured by the APS and by the MOUDI revealed a slight shift in the mass-weighted distributions. For example, size distributions of the $5.1 \mu \mathrm{m}$ polystyrene DVB standard resulted in a major peak located at $5.425 \mu \mathrm{m}$ for the APS and in the 6.2-9.8 $\mathrm{\mu m}$ size range for the MOUDI (Figure 5.2). A similar shift can be seen for the aluminum oxide particles $(0.5-20 \mu \mathrm{m})$, shown in Figure 5.3 . Overloading of the MOUDI substrates was not found to be a problem as similar size distributions were observed for a wide range of impacted material mass on a single stage, up to $8.094 \mathrm{mg}$. At least three possible reasons could account for the apparent discrepancy: (i) the counting efficiencies of the APS 3320 vary with particle size,, 39 (ii) the MOUDI directly measures mass, whereas the APS calculates mass based upon density (measured with a pycnometer), and (iii) the estimated cut-sizes of the MOUDI are not entirely accurate.

With the aluminum silicate test sample (Figure 5.4), the MOUDI and APS appear to be in agreement, with a majority of particles measuring just above $1 \mu \mathrm{m}$ in diameter. However, large particles $>10 \mu \mathrm{m}$ were observed with the APS but not with the MOUDI. These large particles detected by the APS are questionable measurements as they should have been collected by the impactor and detected by gravimetric analysis. The discrepancy between the APS and MOUDI could be due to a few erroneous large particle measurements made by the APS when small particles $(<5 \mu \mathrm{m})$ are inadvertently recirculated back to the acceleration nozzle. The presence of only a few false large particles can have a significant effect on the mass-weighted size distribution measurements causing the discrepancies between the APS and MOUDI. Armendariz and Leith ${ }^{39}$ have shown that artificial large particle counts $(>5 \mu \mathrm{m})$ caused by recirculation within the detection region distorted the shape of the size distribution reported by the APS 3320. In a later study by Peters and Leith, $\frac{40}{1}$ size distributions of an APS model 3321 were compared to that of model 3320 and a cascade impactor. The APS 3321 had lower counting efficiencies than the APS 3320, but the size distribution was similar to that obtained using the impactor.

Comparative size distributions for the APS and MOUDI for BG spores are summarized on a mass-weighted basis in Figure 5.5. APS measured size distributions for $\mathrm{BG}$ spores were in fair agreement with those of the MOUDI. BG aerosol particles generated by the SSPD and analyzed by the APS revealed aerodynamic size measurements centered on $7.774 \mu \mathrm{m}$. Similarly, the MOUDI collected the most mass between 6.2-17.7 $\mu \mathrm{m}$. The mass-weighted size distributions of the APS and MOUDI indicate that a number of particles are not individual spores (sample is not 'weaponized'). 

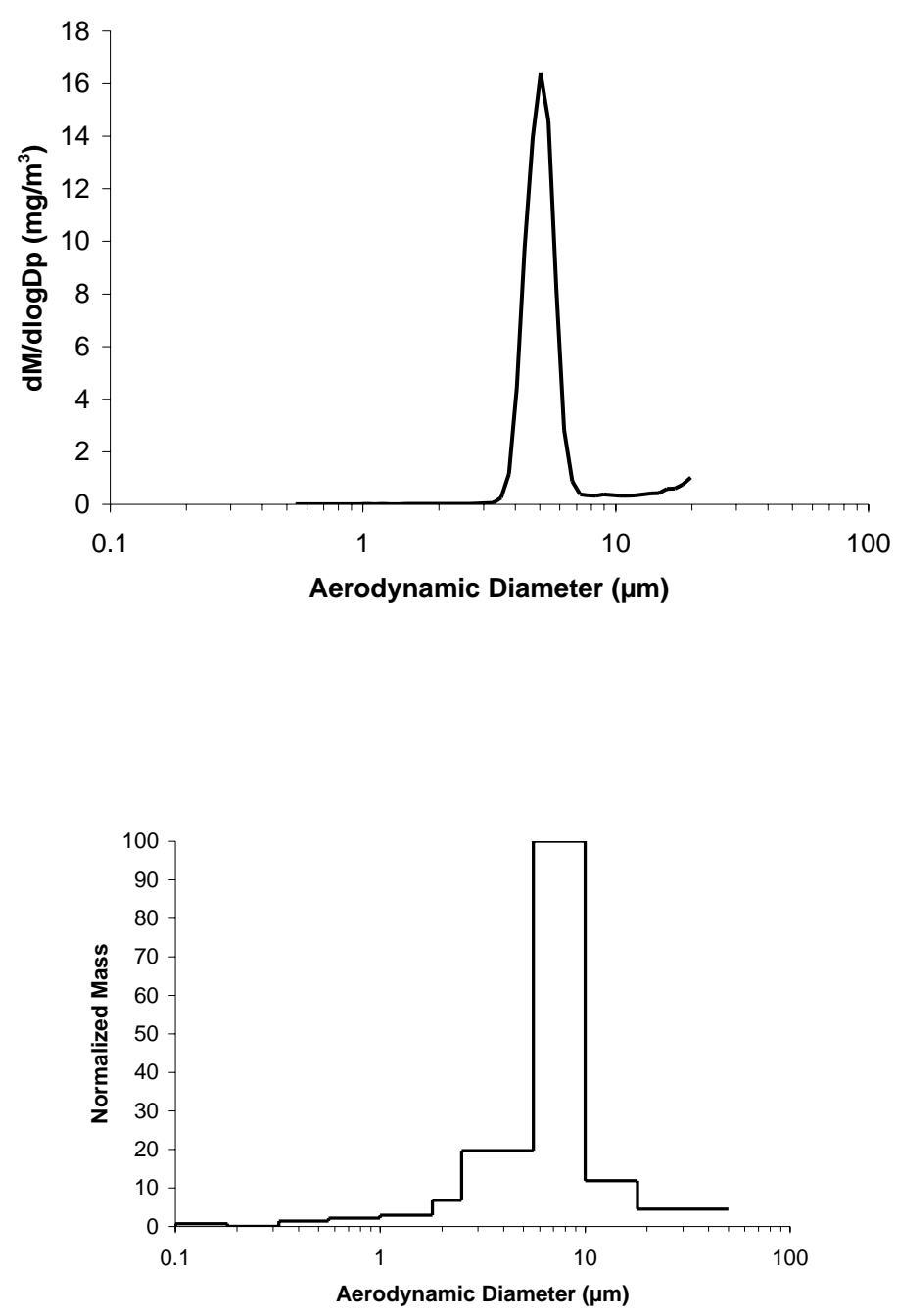

Figure 5.2: Average mass-weighted size distributions for polystyrene DVB microspheres: (Top) APS, (Bottom) MOUDI. 

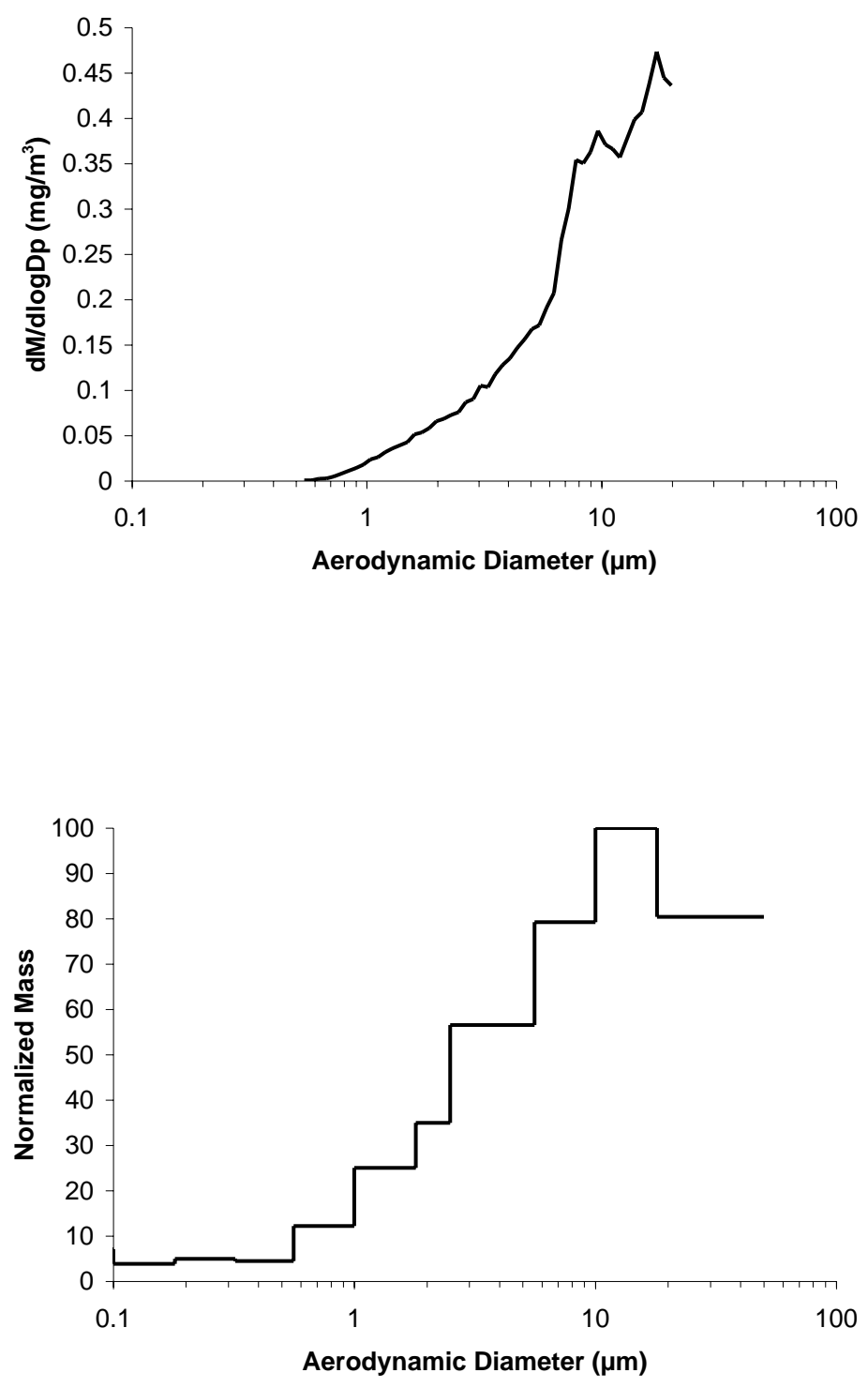

Figure 5.3: Average mass-weighted size distributions for aluminum oxide particles: (Top) APS, (Bottom) MOUDI. 

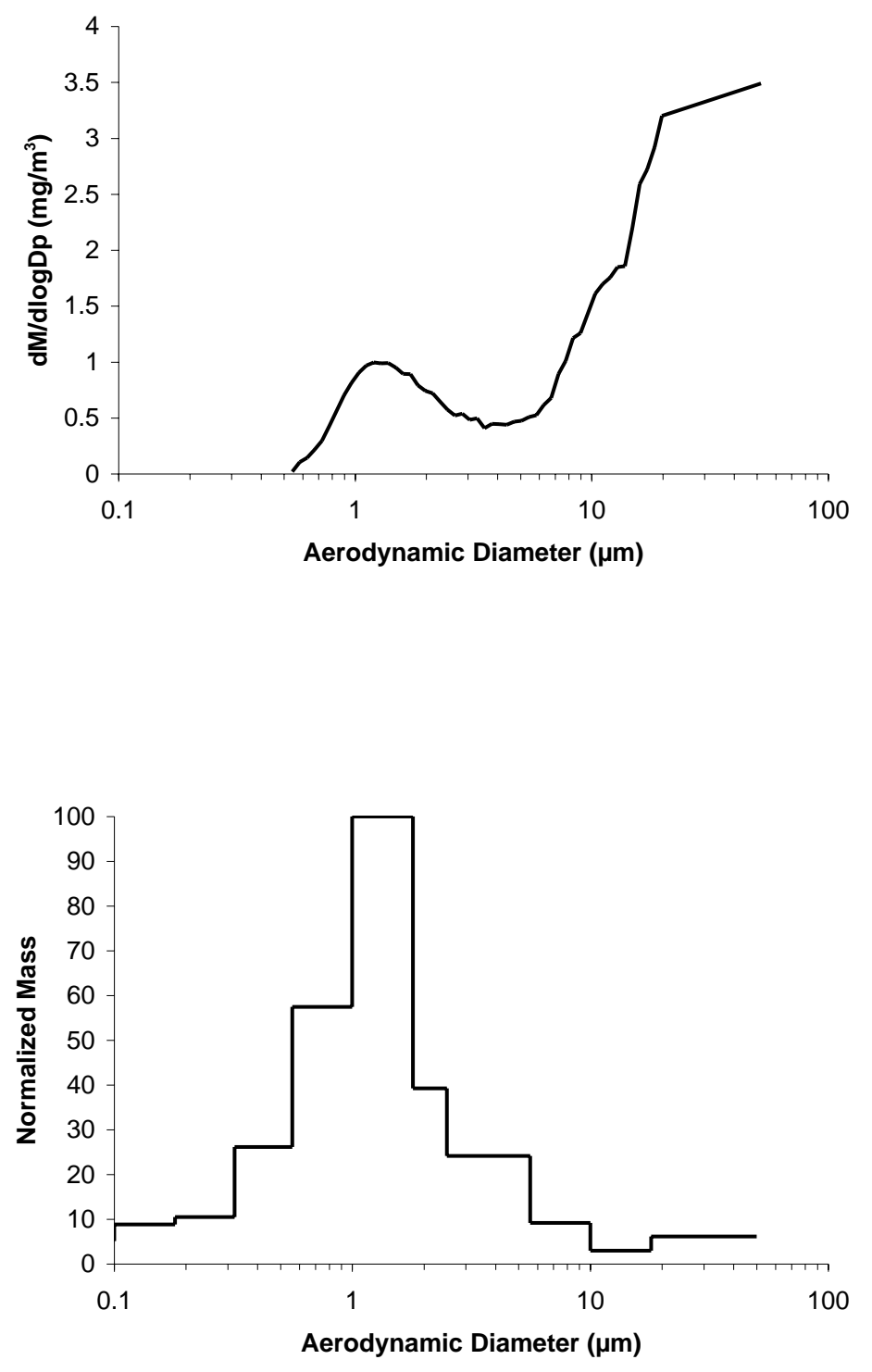

Figure 5.4: Average mass-weighted size distributions for aluminum silicate particles: (Top) APS, (Bottom) MOUDI. 
It is more likely that the large particles are of Bacillus spores embedded in growth media and/or bound together forming aggregates of a few microns in size.

Gravimetric analysis of the MOUDI's ten impaction stages compare favorably with the mass-weighted size distributions of the APS using in conjunction with a pycnometer. The APS is more convenient and rapid than the MOUDI, allowing for the testing of a large number of samples with a number of replicates.

\subsubsection{Morphologic analysis of Bacillus globigii spores}

Microbial characterization begins with their physical appearance. Physical characteristics (overall shape, size, detailed surface features, as well as color) can aid in fingerprinting the biological agent. Anthrax spores are rod-shaped with diameters of 2-6 microns ${ }^{41}$ the ideal size for respiration. Figure 5.6 shows SEM micrographs of the ten MOUDI stages under 15,000x magnification. The SEM micrographs easily confirm the presence of spores that are rod-like in shape, $\sim 1.45 \mu \mathrm{m}$ in length and $\sim 0.65 \mu \mathrm{m}$ in width. Although it appears that some of the particles are too large to have impacted onto the correct MOUDI stage, it is possible that particles impacted adjacent to each other or once impacted, electrostatically moved to each other.

A close look at the media surrounding the spores can illustrate what growth and processing method was performed. Variations in particle morphology can differentiate between the 'weaponization' processes used to generate the samples, whereas the degree of post growth processing can be an indicator of the sophistication of the terrorist operation. One method to inhibit clumping and produce weapons-grade spores is to coat them with something that interrupts the weak van der Waals interactions between each particle, such as silica. ${ }^{42}$ Using SEM, Schaldach et al.21 presented images of bacterial spores that illustrated the visual differences of postgrowth processes to improve 'weaponization': bacterial spores with clumps of silica, embedded in fumed silica, coated with silica, and coated with colloidal silica particles. Comparison of these micrographs with the ones of Figure 5.6, indicates that post-growth processing with silica was not performed on this particular sample.

Separating the particulate sample into size fractions prior to SEM analysis is beneficial. Bacillus spores can be seen embedded in what is believed to be growth media, as in MOUDI stage 2, while MOUDI stage 6 shows individual BG spores (indicated by the arrows) impacted onto the foil substrate. Individual spores, amenable to surface characterization by SEM, can more easily be found on the correct MOUDI impaction stage than scanning a bulk sample 'hunting' for spores. This becomes 

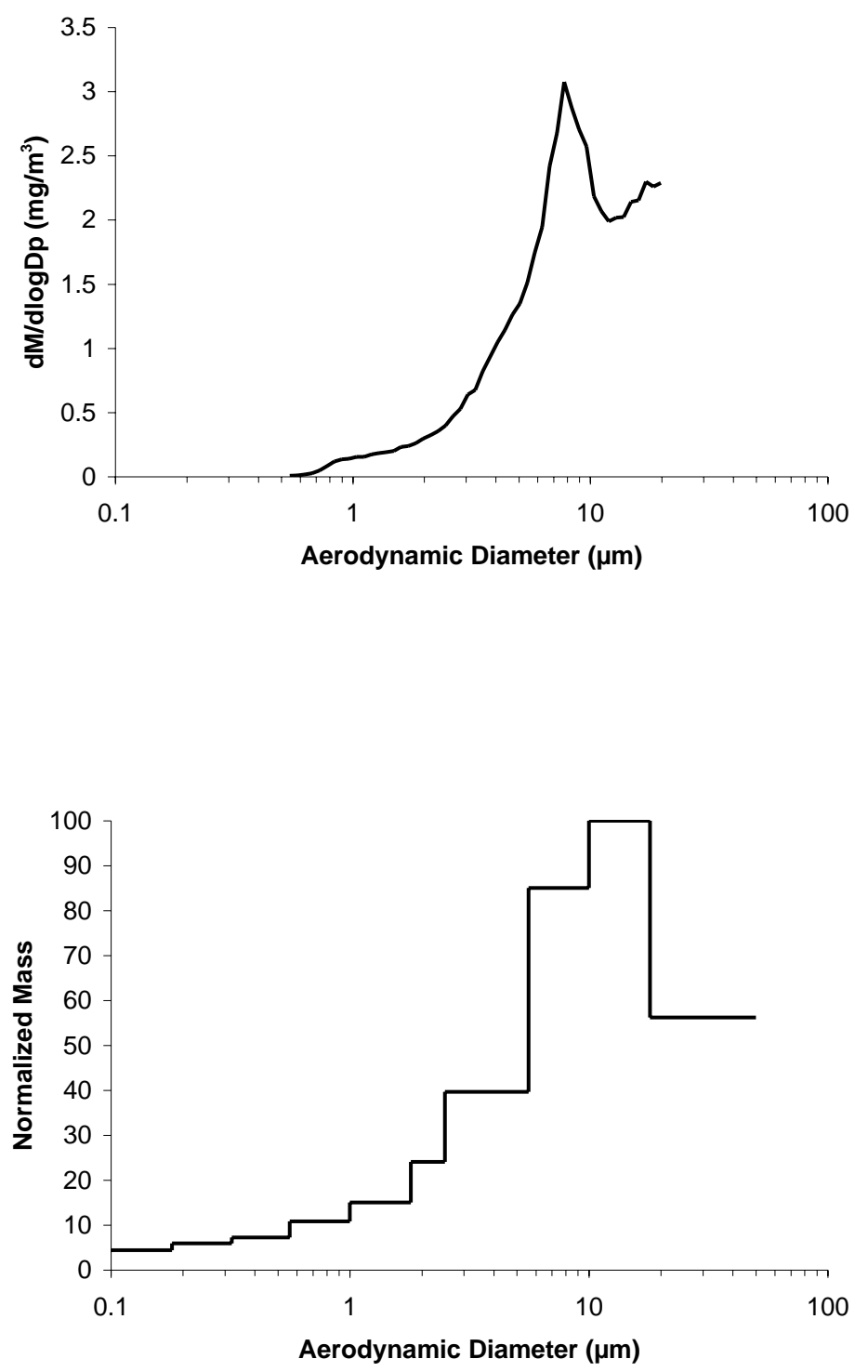

Figure 5.5: Average mass-weighted size distributions for BG spores: (Top) APS, (Bottom) MOUDI. 


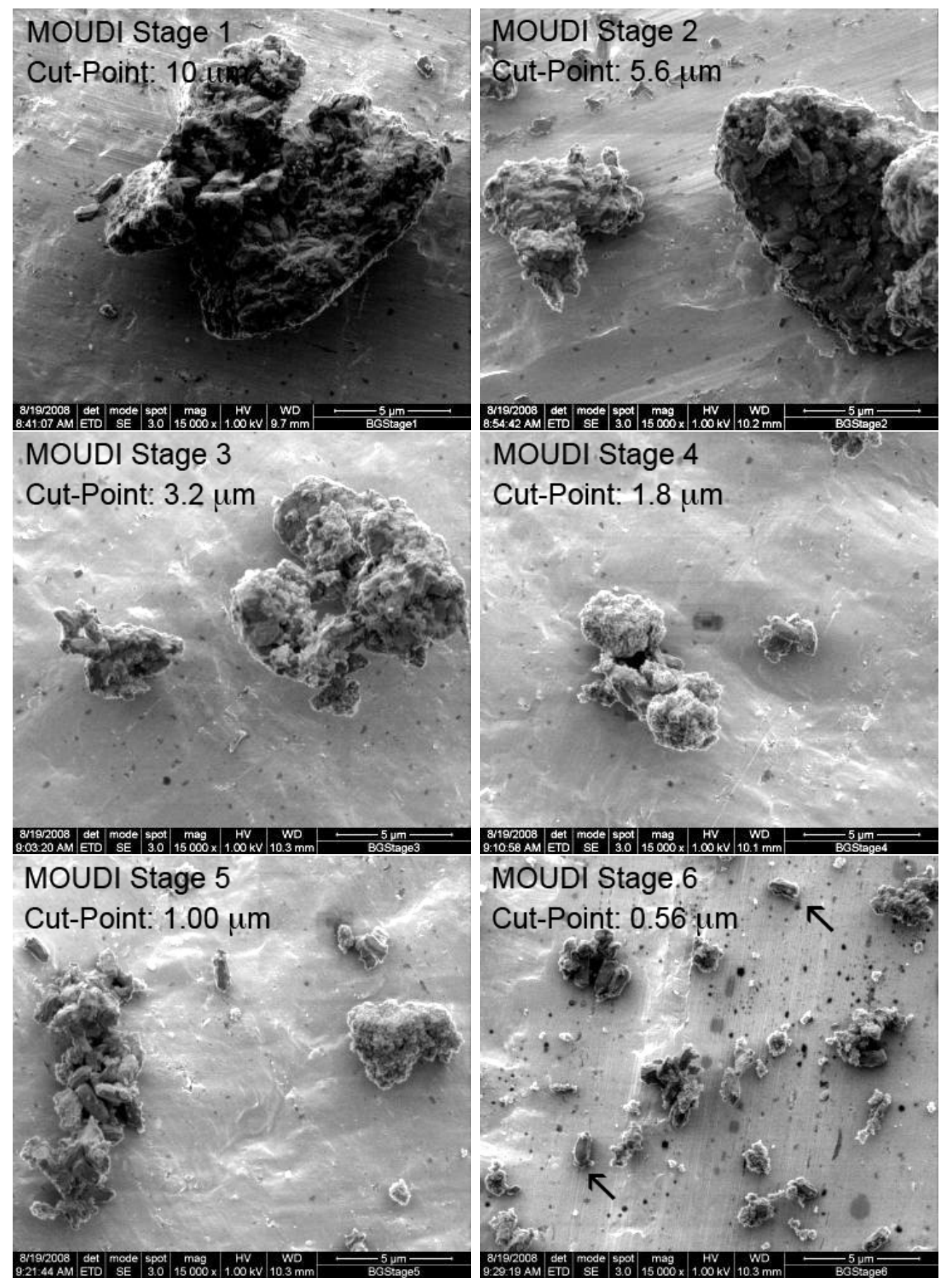




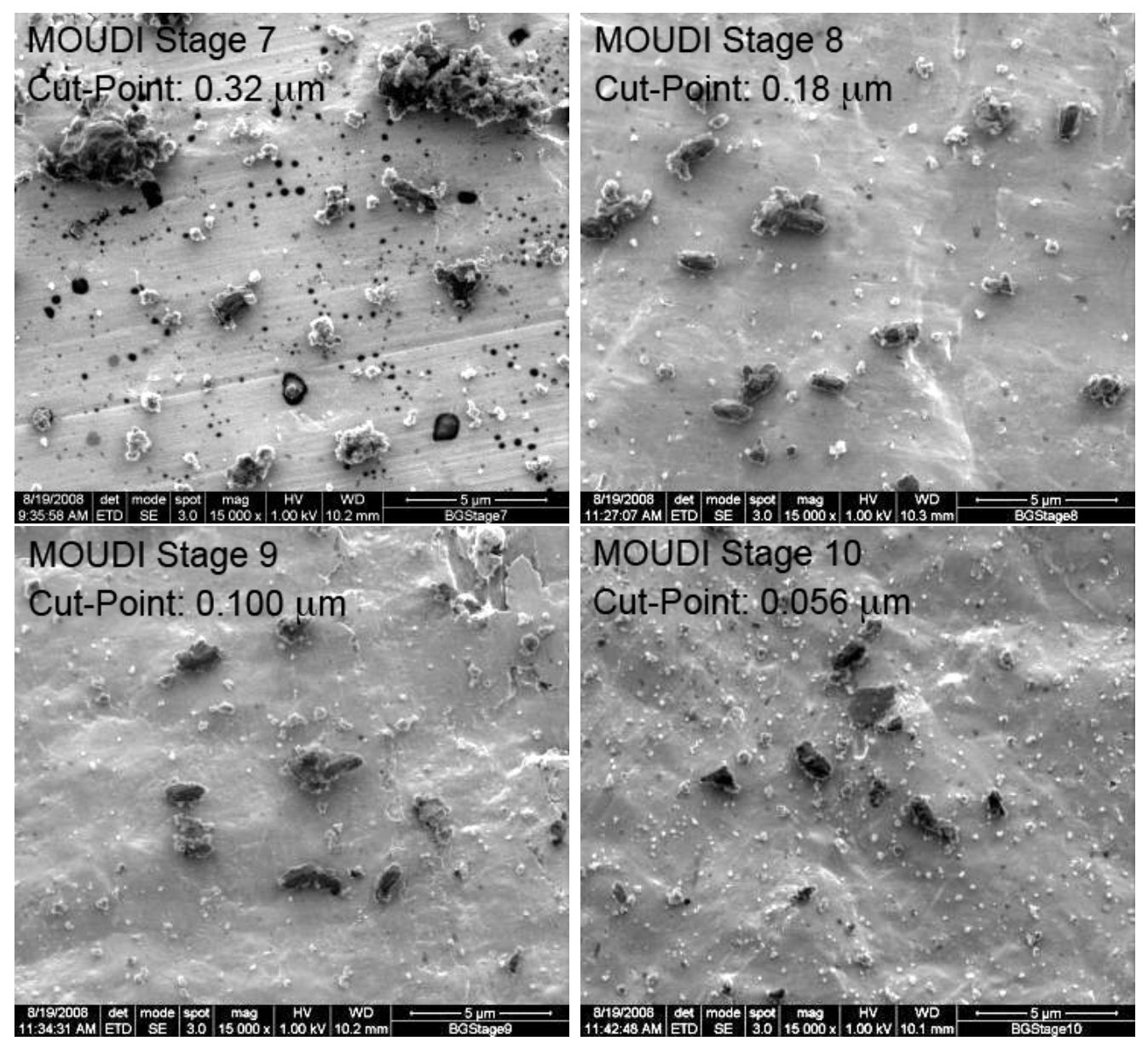

Figure 5.6: Scanning electron micrograph images of uncoated B. globigii spores. The powdered material was size-fractionated by the MOUDI onto aluminum foil substrates prior to SEM analysis. 
especially important when samples are not homogeneous and/or of low spore concentrations. From the micrographs, it is clear that the particulate sample is composed of a low spore concentration and clumping can be seen. However, biological agents do not need to be elegantly weaponized to be lethal or for effective dissemination. 43 Although the material analyzed is not viable for use as a small-particle aerosol for entrance into a victim's lungs, it is plausible to infect populations by deliberate contamination of the country's food supply.

\subsubsection{Molecular composition}

Raman spectra reveal the intrinsic information on the molecular composition and structure of a sample by providing a distinct 'fingerprint' that allows for the rapid identification of the material under analysis. The performance of the R-3000 Raman spectrometer was first tested using different samples of polystyrene: a polystyrene coffee cup, polystyrene powder in a glass vial, and polystyrene powder dispersed onto a foil substrate (Figure 5.7). Comparing the samples, it is clear that the R-3000 is capable of producing spectra of particulate material on a foil substrate strikingly similar to spectra taken from a bulk sample.

Figure 5.8 shows Raman spectra of (a) blank foil substrates, (b) Bacillus globigii spores size-fractionated by the MOUDI onto foil substrates, and (c) a bulk sample of BG spores housed in a glass vial. Following fractionation by the MOUDI, the spores do not distribute evenly across the foil substrate. To ease this issue, spectra of the MOUDI size-fractionated samples were collected at three different locations on the substrate and the one of highest intensity is shown. Results show that as the amount of sample impacted onto the foil substrate increases, the Raman spectra resemble less like the foil substrate and more like that of the BG bulk sample. In both the spectra of bulk sample and the upper MOUDI stages, a broad hump can be seen between 1000 and $1030 \mathrm{~cm}^{-1}$. This may or may not be a feature associated with calcium dipicolinate (CaDPA). Raman spectra of spore-forming bacteria have shown to be dominated by $\mathrm{CaDPA}^{25144}$ with its primary peak at $1017 \mathrm{~cm}^{-1}$; however, those spectra were of pure spores.

Unfortunately, size-fractionation of the BG particulate sample prior to Raman analysis did not appear to provide any benefit. From the SEM micrographs discussed earlier in this chapter, it was concluded that the single broad-shaped Raman signal can be attributed to the media surrounding the spores. 


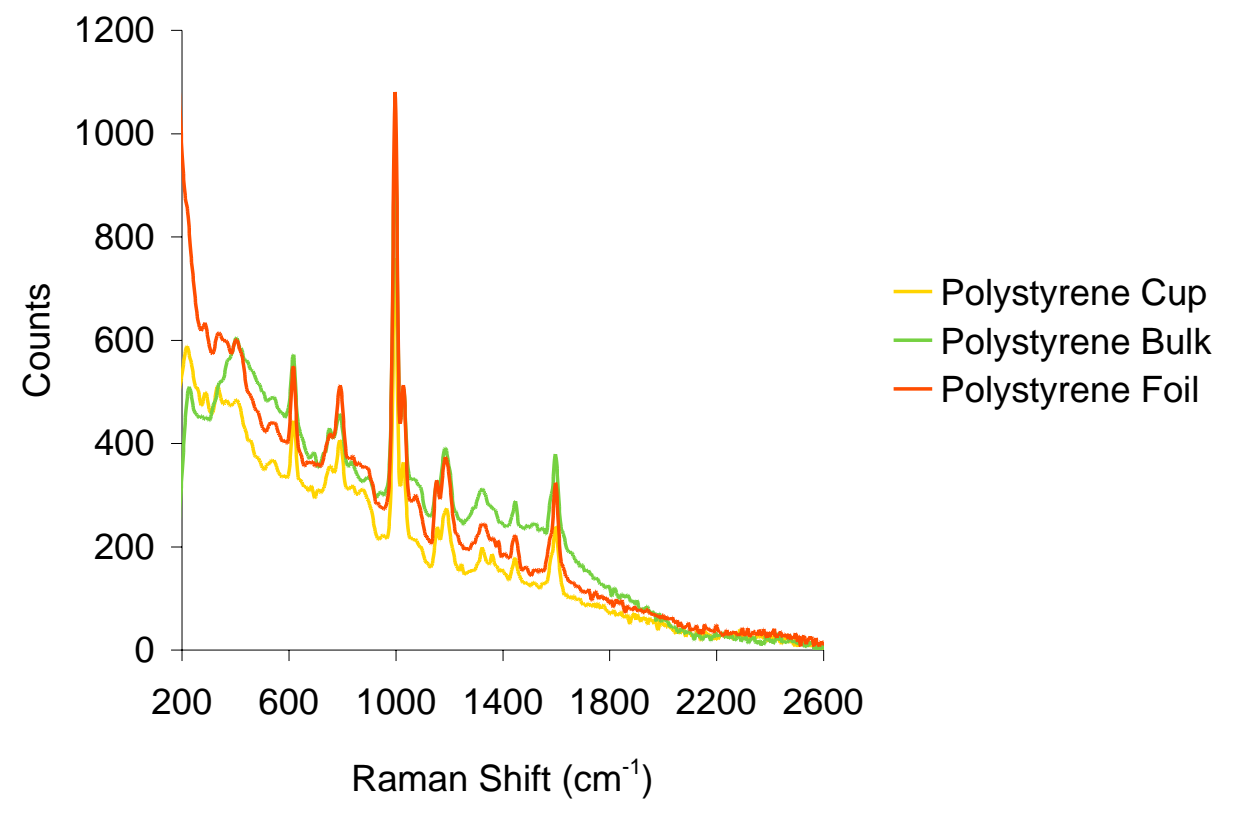

Figure 5.7: Raman spectra of a polystyrene cup, polystyrene bulk powder in a glass vial, and polystyrene powder dispersed onto a MOUDI foil substrate. 


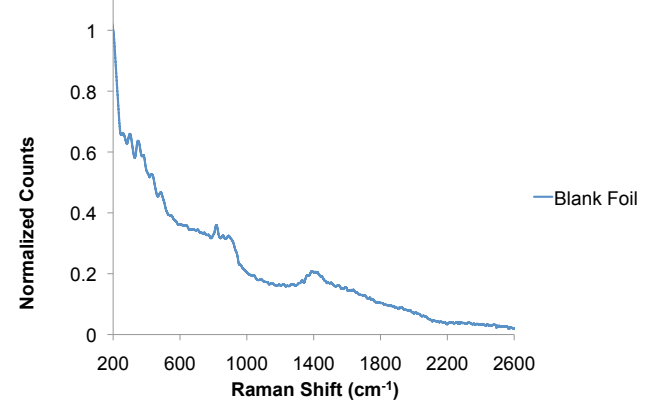

(a) Blank foil substrates

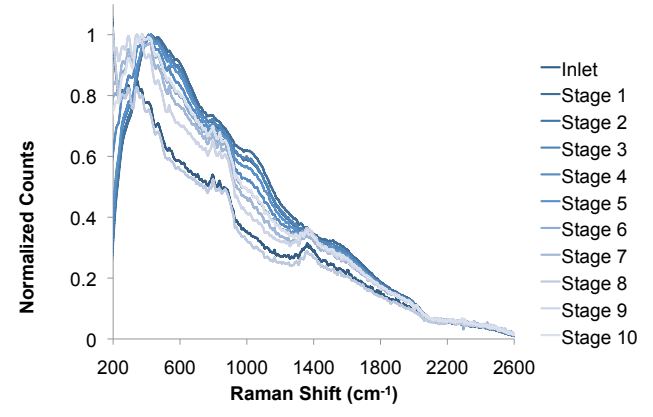

(b) Size-fractionated BG spore sample on foil substrates

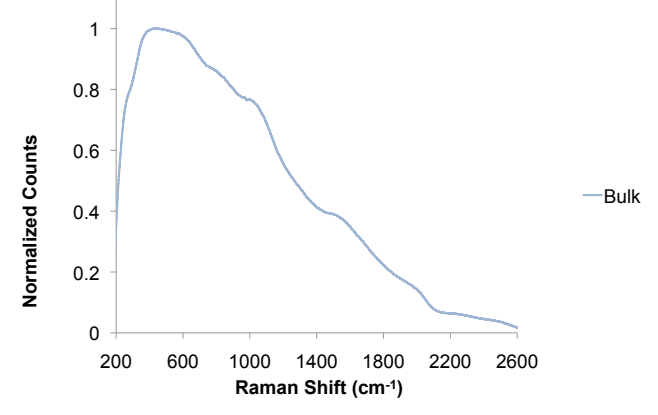

(c) Bulk sample of BG spores

Figure 5.8: Raman spectra of (a) blank foil substrates, (b) size-fractionated BG spore sample on foil substrates, and (c) bulk sample of BG spores. 


\subsection{Conclusions and future directions}

Experimental data is presented to illustrate the application of size fractionation in combination with a series of complementary techniques in order to probe deeper for biological signatures of bacterial spores. These signatures reflect the growth and processing history of the organism and are important for creating libraries for geolocation and attribution purposes.

The micro-orifice uniform deposit impactor was first compared to an aerodynamic particle sizer using three different particle size standards. The APS 3320 provided mass-distribution measurements that correlated well with the more traditional cascade impactor, but the less-time consuming and higher-resolution APS spectrometer has proved to be an ideal tool for the analysis of a particulate sample's size distribution pattern. The mean number-weighted and mass-weighted aerodynamic diameter were found to be $0.835 \mu \mathrm{m}$ and $7.774 \mu \mathrm{m}$ (well above the size of a single spore), respectively. The APS measured mass-weighted size distribution alone tells us that the BG particulate sample consists of large particulates of both media and spores, suggesting minimal processing.

When spore concentrations are low, size fractions ease the process of locating individual spores for SEM imaging. The MOUDI cascade impactor fractionated the BG particulate sample by size onto 10 stages plus an inlet stage. Following size fractionation, SEM analysis confirmed the spores were rod-shaped (i.e. Bacillus spores) and the majority of the sample was not composed of individual spores (i.e. of low spore concentration). The results from SEM micrographs also led us to conclude the spores were not coated with silica (i.e. not weapons-grade). Further analysis by ICP-MS should be able to confirm/refute this finding. Raman and surface-enhanced Raman spectroscopy produced nearly identical spectra between the bulk BG sample and MOUDI size fractions. Although prior knowledge of the microbial sample is unknown and this work does not include a comparison of results with Bacillus spores from other manufacturing processes, information is still gained as to the nature of the sample and this work demonstrates that particulate characterization can benefit from size fractionation.

Analysis of microbial material is capable of answering a number of questions such as where, when, and how the sample was produced. Characterization includes properties such as size, shape, and physical morphology, in addition to full elemental analysis, including major, minor, and trace constituents. Future direction will focus on (i) integrating this approach with other techniques, such as ICP-MS, SEM with energy dispersive X-ray analysis, and bio-aerosol mass spectrometry, (ii) use of 
the APS 3321, and (iii) extending the analysis to a wider range of growth media, processing methods, and organisms. 


\section{Bibliography}

[1] C. A. Hart, Microterrors: The Complete Guide to Bacterial, Viral and Fungal Infections that Threaten Osur Health. Firefly Books Ltd., 2004.

$[2]<$ http://www.bt.cdc.gov/agent/anthrax/needtoknow.asp $>$ accessed 2 July 2009.

[3] T. H. Rider, M. S. Petrovick, F. E. Nargi, J. D. Harper, E. D. Schwoebel, R. H. Mathews, D. J. Blanchard, L. T. Bortolin, A. M. Young, J. Chen, and M. A. Hollis, "A B cell-based sensor for rapid identification of pathogens," Science, vol. 301, pp. 213-215, 2003.

[4] D. J. Ecker, R. Sampath, L. B. Blyn, M. W. Eshoo, C. Ivy, J. A. Ecker, B. Libby, V. Samant, K. A. Sannes-Lowery, R. E. Melton, K. Russell, N. Freed, C. Barrozo, J. Wu, K. Rudnick, A. Desai, E. Moradi, D. J. Knize, D. W. Robbins, J. C. Hannis, P. M. Harrell, C. Massire, T. A. Hall, Y. Jiang, R. Ranken, J. J. Drader, N. White, J. A. McNeil, S. T. Crooke, and S. A. Hofstadler, "Rapid identification and strain-typing of respiratory pathogens for epidemic surveillance," Proceedings of the National Academy of Sciences of the United States of America, vol. 102, no. 22, pp. 8012-8017, 2005.

[5] T. C. Marentis, B. Kusler, G. G. Yaralioglu, S. Liu, E. O. Haeggstrom, and B. T. Khuri-Yakub, "Microfluidic sonicator for real-time disruption of eukaryotic cells and bacterial spores for DNA analysis," Ultrasound in Medicine and Biology, vol. 31, no. 9, pp. 1265-1277, 2005.

[6] Y. W. Tang and C. W. Stratton, eds., Advanced Techniques in Diagnostic Microbiology. Springer-Verlag LLC, 2006.

[7] W. C. Schumacher, C. A. Storozuk, P. K. Dutta, and A. J. Phipps, "Identification and characterization of Bacillus anthracis spores by multiparameter flow cytometry," Applied and Environmental Microbiology, vol. 74, no. 16, pp. 52205223, 2008.

[8] C. Fenselau and P. Demirev, "Characterization of intact microorganisms by MALDI mass spectrometry," Mass Spectrometry Reviews, vol. 20, p. 157, 2001.

[9] J. Lay, "MALDI-TOF mass spectrometry of bacteria," Mass Spectrometry Reviews, vol. 20, no. 172, 2001. 
[10] J. P. Dworsanski and P. A. Snyder, "Classification and identification of bacteria using mass spectrometry-based proteomics," Expert Review of Proteomics, vol. 2, p. 863, 2005 .

[11] C. L. Wilkins and J. O. Lay Jr., eds., Identification of Miicroorganisms by Mass Spectrometry. John Wiley \& Sons, Ltd., 2006.

[12] P. A. Demirev and C. Fenselau, "Mass spectrometry in biodefense," Journal of Mass Spectrometry, vol. 43, pp. 1441-1457, 2008.

[13] C. M. Gikunju, S. M. Lev, A. Birenzvige, and D. M. Schaefer, "Detection and identification of bacteria using direct injection inductively coupled plasma mass spectroscopy," Talanta, vol. 62, pp. 741-744, 2004.

[14] A. Srivastava, M. E. Pitesky, P. T. Steele, H. J. Tobias, D. P. Fergenson, J. M. Horn, S. C. Russell, G. A. Czerwieniec, C. B. Lebrilla, E. E. Gard, and M. Frank, "Comprehensive assignment of mass spectral signatures from individual Bacillus atrophaeus spores in matrix-free laser desorption/ionization bioaerosol mass spectrometry," Analytical Chemistry, vol. 77, no. 10, pp. 3315-3323, 2005.

[15] W. H. Griest and S. A. Lammert, Identification of Microorganisms by Mass Spectrometry, ch. 4: The Development of the Block II Chemical Biological Mass Spectrometer. John Wiley \& Sons, Ltd., 2006.

[16] P. T. Steele, G. R. Farquar, A. N. Martin, K. R. Coffee, V. J. Riot, S. I. Martin, D. P. Fergenson, E. E. Gard, and M. Frank, "Autonomous, broadspectrum detection of hazardous aerosols in seconds," Analytical Chemistry, vol. 80, no. 4583-4589, 2008.

[17] <http://www.merriam-webster.com/dictionary/attribution > accessed 6 July 2009.

[18] B. Budowle, S. E. Schutzer, A. Einseln, L. C. Kelley, A. C. Walsh, J. A. L. Smith, B. L. Marrone, J. Robertson, and J. Campos, "Building microbial forensics as a response to bioterrorism," Science, vol. 301, p. 1852, 2003.

[19] J. R. Whiteaker, C. Fenselau, D. Fetterolf, D. Steele, and D. Wilson, "Quantitative determination of heme for forensic characterization of Bacillus spores using matrix-assisted laser desorption/ionization time-of-flight mass spectrometry," Analytical Chemistry, vol. 76, p. 2836, 2004. 
[20] R. Breeze, B. Budowle, and S. E. Schutzer, Microbial Forensics. Elsevier Inc., 2005.

[21] C. M. Schaldach, G. Bench, J. J. DeYoreo, T. Esposito, D. P. Fergenson, J. Ferreira, E. Gard, P. Grant, C. Hollars, J. Horn, T. Huser, M. Kashgarian, J. Knezovich, S. M. Lane, A. J. Malkin, M. Pitesky, C. Talley, H. J. Tobias, B. Woods, K.-J. Wu, and S. P. Velsko, Microbial Forensics, ch. 13: Non-DNA Methods for Biological Signatures. Elsevier Inc., 2005.

[22] H. W. Kreuzer-Martin and K. H. Jarman, "Stable isotope ratios and forensic analysis of mircroorganisms," Applied and Environmental Microbiology, vol. 73, no. 12, pp. 3896-3908, 2007.

[23] R. Wang, S. N. Krishnamurthy, J.-S. Jeong, A. Driks, M. Mehta, and B. A. Gingras, "Fingerprinting species and strains of bacilli spores by distinctive coat surface morphology," Langmuir, vol. 23, pp. 10230-10234, 2007.

[24] H. Shibata, "Laser Raman spectroscopy of lyophilized bacterial spores," Microbiol Immunol, vol. 30, pp. 307-313, 1986.

[25] S. Farquharson, L. Grigely, V. Khitrov, W. Smith, J. F. Sperry, and G. Fenerty, "Detecting Bacillus cereus spores on a mail sorting system using Raman spectroscopy," Journal of Raman Spectroscopy, vol. 35, no. 82-86, 2004.

[26] J. W. Chan, A. P. Esposito, C. E. Talley, C. W. Hollars, S. M. Lane, and T. Huser, "Reagentless identification of single bacterial spores in aqueous solution by confocal laser tweezers Raman spectroscopy," Analytical Chemistry, vol. 76, no. 3, pp. 599-603, 2004.

[27] J. De Gelder, P. Scheldeman, K. Leus, M. Heyndrickx, P. Vandenabeele, L. Moens, and P. De Vos, "Raman spectroscopic study of bacterial endospores," Analytical Bioanalytical Chemistry, vol. 389, pp. 2143-2151, 2007.

[28] E. Ghiamati, "UV resonance Raman spectra of Bacillus spores," Appl Spectrosc, vol. 46, pp. 357-364, 1992.

[29] A. P. Esposito, C. E. Talley, and T. Huser, "Analysis of single bacterial spores by micro-Raman spectroscopy," Appl Spectrosc, vol. 57, pp. 868-871, 2003.

[30] B. Kournikakis, S. J. Armour, C. A. Boulet, M. Spence, and B. Parsons, "Risk assessment of anthrax threat letters," technical report, Defence Research Establishment Suffield, 2001. 
[31] D. N. Stratis-Cullum, G. D. Griffin, J. Mobley, A. A. Vass, and T. Vo-Dinh, "A miniature biochip system for detection of aerosolized bacillus globigii spores," Analytical Chemistry, vol. 75, pp. 275-280, 2003.

[32] S. Farrell, H. B. Halsall, and W. R. Heineman, "Bacillus globigii bugbeads: a model simulant of a bacterial spore," Analytical Chemistry, vol. 77, pp. 549-555, 2005 .

[33] K. D. Chichester, D. B. Silcott, and C. L. Colyer, "Analysis of Bacillus globigii spores by CE," Electrophoresis, vol. 29, pp. 641-651, 2008.

[34] S. K. Mwilu, A. O. Aluoch, S. Miller, P. Wong, and O. A. Sadik, "Identification and quantitation of Bacillus globigii using metal enhanced electrochemical detection and capillary biosensor," Analytical Chemistry, vol. 81, pp. 7561-7570, 2009 .

[35] V. A. Marple, K. L. Rubow, and S. M. Behm, "A microorifice uniform deposit impactor (MOUDI): description, calibration, and use," Aerosol Science and Technology, vol. 14, pp. 434-446, 1991.

[36] Personal communication with Francisco Romay of MSP Corp regarding MOUDI cut-points.

[37] G. Rudolph, R. Kobrich, and W. Stahlhofen, "Modeling and algebraic formulation of regional aerosol deposition in man," Journal of Aerosol Science, vol. 21, pp. 306-406, 1990.

[38] P. P. Hairston, J. Ho, and F. R. Quant, "Design of an instrument for real-time detection of bioaerosols using simultaneous measurement of particle aerodynamic size and intrinsic fluorescence," Journal of Aerosol Science, vol. 28, no. 3, pp. 471-482, 1997.

[39] A. J. Armendariz and D. Leith, "Concentration measurement and counting efficiency for the aerodynamic particle sizer 3320," Journal of Aerosol Science, vol. 33, no. 1, pp. 133-148, 2001.

[40] T. M. Peters and D. Leith, "Concentration measurement and counting efficiency of the aerodynamic particle sizer 3321," Journal of Aerosol Science, vol. 34, no. 5, pp. 627-634, 2003.

[41] T. J. Cieslak and E. M. Eitzen, Jr., "Clinical and epidemiologic principles of anthrax," Emerging Infectious Diseases, vol. 5, no. 4, pp. 552-555, 1999. 
[42] G. Matsumoto, "Anthrax powder: State of the art?," Science, vol. 302, pp. 14921497, 2003.

[43] Committee on Science and Technology for Countering Terrorism, National Research Council, Making the nation safer: the role of science and technology in countering terrorism. The National Academies Press, 2002.

[44] W. H. Nelson, R. Dasari, M. Feld, and J. F. Sperry, "Intensities of calcium dipicolinate and Bacillus subtilis spore Raman spectra excited with $244 \mathrm{~nm}$ light," Applied Spectroscopy, vol. 58, pp. 1408-1412, 2004. 


\section{Abbreviations and Acronyms}

APS Aerodynamic Particle Sizer

Ar Argon

BA Bacillus anthracis

BG Bacillus globigii

C Carbon

CDC Centers for Disease Control and Prevention

$\mathrm{CO}_{2}$ Carbon Dioxide

$\mathrm{Cu}$ Copper

CWA Chemical Warfare Agent

$D$ Distance

dc Direct Current

DDG Digital Delay Generator

DIP Direct Insertion Probe

$e$ Charge on an Electron

$E \quad$ Electric Field

EI Electron Ionization

EPA Environmental Protection Agency

FEG Field Emission Gun

FWHM Full Width at Half Maximum

GC Gas Chromatography

GD Glow Discharge

$\mathrm{H}_{2} \mathrm{O}$ Water

HPLC High-Performance Liquid Chromatography

ICP Inductively Coupled Plasma

i.d. Internal Diameter

IMPA Isopropyl Methyl Phosphonic Acid

IP Ionization Potential

KE Kinetic Energy

LTE Local Thermodynamic Equilibrium

$m$ Mass

MCP Microchannel Plate

$\mathrm{mm}$ Millimeter

MOUDI Micro-Orifice Uniform Deposit Impactor

MPA Methyl Phosphonic Acid

MS Mass Spectrometry 
MTBSTFA N-methyl-N-(tert-butyldimethylsilyl)-trifluoroacetamide

$m / z$ Mass to Charge Ratio

$\mathrm{N}$ Nitrogen

O Oxygen

PDMS Polydimethylsiloxane

rf Radio Frequency

$R$ Resolution

SEM Scanning Electron Microscope

SPME Solid Phase Micro-Extraction

SSPD Small Scale Powder Disperser

$t$ Time

TOF Time-of-Flight

$\mu \mathrm{m}$ Micrometer

$\mu \mathrm{s} \quad$ Microsecond

$v$ Velocity

$z \quad$ Number of Charges on an Ion 